University of Rhode Island

DigitalCommons@URI

Open Access Master's Theses

2007

\title{
Modeling, Simulation and Control of a Belt Driven, Parallel H- Frame Type Two Axes Positioning System
}

Klaus S. Sollmann

University of Rhode Island

Follow this and additional works at: https://digitalcommons.uri.edu/theses

\section{Recommended Citation}

Sollmann, Klaus S., "Modeling, Simulation and Control of a Belt Driven, Parallel H-Frame Type Two Axes Positioning System" (2007). Open Access Master's Theses. Paper 607.

https://digitalcommons.uri.edu/theses/607

This Thesis is brought to you for free and open access by DigitalCommons@URI. It has been accepted for inclusion in Open Access Master's Theses by an authorized administrator of DigitalCommons@URI. For more information, please contact digitalcommons-group@uri.edu. 
MODELING, SIMULATION AND CONTROL OF A BELT DRIVEN, PARALLEL H-FRAME TYPE TWO AXES POSITIONING SYSTEM BY KLAUS S. SOLLMANN

A THESIS SUBMITTED IN PARTIAL FULFILLMENT OF THE REQUIREMENTS FOR THE DEGREE OF MASTER OF SCIENCE

IN

MECHANICAL ENGINEERING AND APPLIED MECHANICS 


\section{MASTER OF SCIENCE THESIS}

OF

KLAUS S. SOLLMANN

\section{APPROVED:}

Thesis Committee:

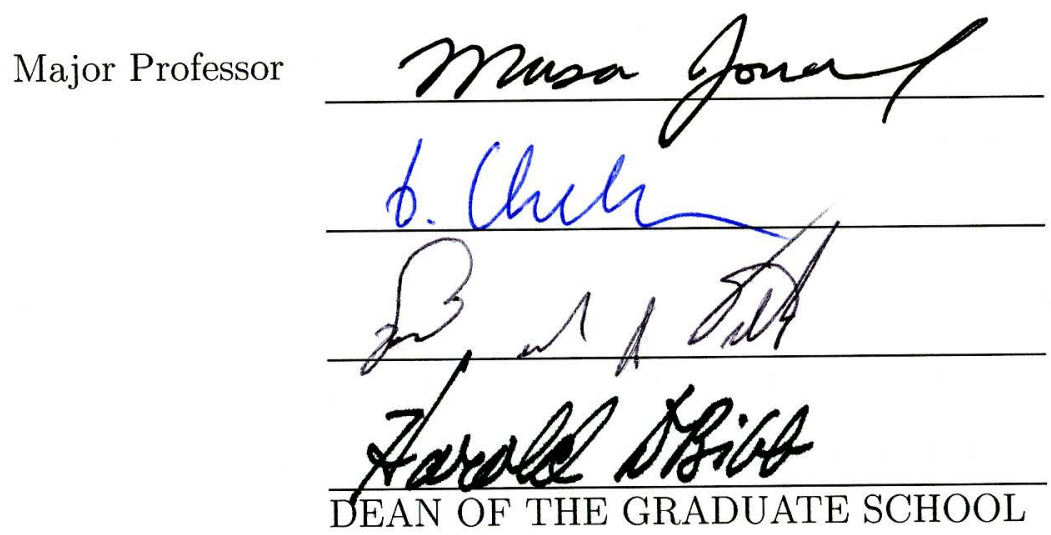




\begin{abstract}
$\mathrm{XY}$-positioning is an important task in industrial applications. The improvement of the speed of positioning while keeping good accuracy can increase productivity and is therefore highly desirable. The studied H-frame system is a parallel xy-positioning device, which due to less moved masses is potentially capable of fast acceleration and therefore faster positioning then traditional stacked systems. One feature of the system which enables its parallel structure is the use of one long timing belt to transmit the power of the two stationary motors to the end-effector stage. The use of an elastic transmission element also causes the biggest disadvantage of the system, an uncertainty of end-effector position, since the end-effector position, for practical as well as economical reasons, is not available as feedback signal for controlling the system. This means that even if an accurate tracking of the desired motor angles was achieved, the end-effector position may still deviate from the desired path, due to stretching in the belt. Another effect making an accurate control difficult is the presence of nonlinear friction in the system. This thesis addresses the development of an accurate control system, which is a requirement to be able to utilize the advantages that the parallel structure of the H-frame system provides.
\end{abstract}

In order to be able to develop an accurate control strategy, the dynamic behavior in the presence of nonlinear friction as well as the flexibility of the belt has to be understood. An accurate dynamic model is needed for the development of most sophisticated controllers. That is why in this work first a one-axis belt drive positioning system is studied and a dynamic model for it is derived. The findings from this simpler one-axis case are then utilized to derive a dynamic model for the two axis $\mathrm{H}$-frame system. First a detailed $20^{\text {th }}$-order model of the system is being derived, which then based on assumptions is simplified to a lower order $8^{\text {th }}$-order 
case. Nonlinear friction is considered for the one-axis as well as for the simplified $8^{\text {th }}$-order H-frame case. Experimental results are utilized to verify that the derived models can resemble the dynamics of the system. The model can be used for simulating different controllers before implementing them on the real system as well as it can be used to develop sophisticated controllers. The model is verified with experimental data from the real system. Furthermore the performance of PIDand PD-controllers are studied in simulation as well as experimentally.

It is found that PID- and PD-controllers, due to the disadvantages the system has described above, are not sufficient control strategies for high-speed tracking. However the dynamic nonlinear model derived in this work gives future researchers the tool to develop more sophisticated controllers, in order to solve the problem of accurate control of the H-frame system. 


\section{ACKNOWLEDGMENTS}

My sincere thanks go to my advisor, Professor Musa Jouaneh. His qualities as an academic teacher and enthusiastic guide have made this research an effective and enjoyable experience.

Thank you to my committee members Proffessor David Chelidze, Professor Manbir Sodhi and Professor Frederick J. Vetter for reviewing my thesis and sharing their expertise.

I would like to acknowledge the effort of everybody involved in the cooperation between the University of Rhode Island and the Technical University of Braunschweig, Germany. I am glad that the efforts of everybody involved in this cooperation gave me the opportunity to make the academic and social experience of studying in the United States.

Lastly, I am thankful for the love and everlasting support of my parents Doris and Ulrich Sollmann. Their support of my dreams as well as the guidance they provide me with to help me reach my goals, is what made this valuable experience at the University of Rhode Island possible for me. I also would like to thank my siblings, my sister Laura and my brothers Martin and Julius, for their love and encouragement. 


\section{TABLE OF CONTENTS}

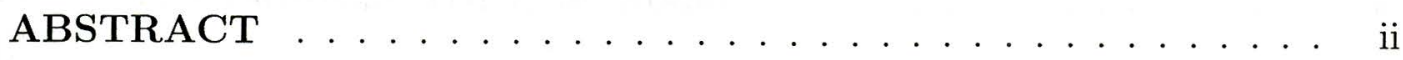

ACKNOWLEDGMENTS ................ iv

TABLE OF CONTENTS $\ldots \ldots \ldots \ldots \ldots \ldots \ldots$

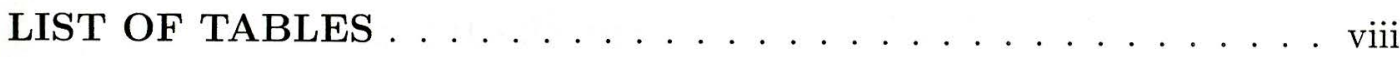

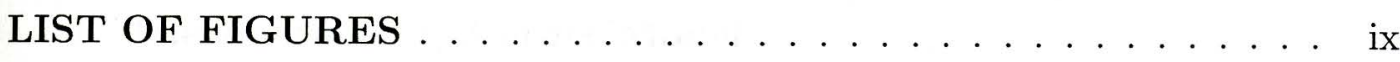

\section{CHAPTER}

1 Introduction . . . . . . . . . . . . . . . 1

1.1 The $\mathrm{H}$-frame positioning system general setup . . . . . . . . 1

1.2 Motivations for the studying of the H-frame system $\ldots \ldots .3$

1.3 Kinematics of the H-frame system . . . . . . . . . . . 6

1.4 Literature Review . . . . . . . . . . . . . . . . . . 7

1.5 Scope of the Thesis . . . . . . . . . . . . . 8

2 The One-Axis Model . . . . . . . . . . . . . . . . . . 10

2.1 Equations of Motion . . . . . . . . . . . . . . 10

2.2 Determination of the Parameters . . . . . . . . . . . 12

$2.2 .1 \quad$ Stiffness $\ldots \ldots \ldots \ldots \ldots \ldots \ldots \ldots$

2.2 .2 Inertia \& Mass $\ldots \ldots \ldots \ldots \ldots$

2.2 .3 Friction . . . . . . . . . . . . . . . . 14

2.3 Simulation of the One-Axis model . . . . . . . . . . . 14

2.4 Nonlinear Model of the One-Axis System . . . . . . . . . 25 
2.5 Discussion of the Results . . . . . . . . . . . . . . . 32

3 The Two-Axes H-frame Model . . . . . . . . . . . . . . 33

3.1 Equations of Motion for the H-frame System . . . . . . . . . . . 33

3.2 Determining the Parameters . . . . . . . . . . . 40

3.3 Verifying the Model by Open Loop Simulation . . . . . . . . . . 40

3.4 Discussion of the Results . . . . . . . . . . . . . . . . 44

4 The simplified 8 th order Model . . . . . . . . . . . 46

4.1 Assumptions to decrease the Order of the Model . . . . . . . . . 46

4.2 Equations of motion for the 8 th order Model . . . . . . . . . . . 49

4.3 Verifying the Model by Open Loop Simulation . . . . . . . . . 53

4.4 Nonlinear Model of the H-frame System . . . . . . . . . . . . . . 57

4.5 Discussion of the Results . . . . . . . . . . . . . 66

4.6 Influence of End-effector XY-position towards Belt Stiffness . . . 66

5 PID-control of the $\mathrm{H}$-frame positioning system . . . . . . . . 70

5.1 Introduction to PID-control of the H-frame System . . . . . . . 70

5.2 Test trajectory . . . . . . . . . . . . . . . 74

5.3 PID simulation setup . . . . . . . . . . . . 78

5.4 PID-controller Performance in Simulation . . . . . . . . . . . . 80

5.4.1 Controller Gains $K_{P}=150, K_{I}=100$ and $K_{D}=4 . . .80$

5.4 .2 Influence of Nonlinear Friction . . . . . . . . . . . . . 82

5.4.3 Influence of Inequality in Motor Errors . . . . . . . . . . 83

5.4.4 PID-controller Final Value Theorem . . . . . . . . . . . . 87

5.4.5 Controller Gains $K_{P}=150, K_{I}=1500$ and $K_{D}=4$. . 90 
5.4.6 Influence of Integral Action on Motor Error Inequality . 92

5.5 PD-controller performance in simulation $\ldots \ldots \ldots . \ldots 93$

5.5.1 PD-controller final value theorem . . . . . . . . . 94

5.5.2 Controller Gains $K_{P}=150$ and $K_{D}=4 \ldots \ldots$. . . 95

5.5.3 Controller Gains $K_{P}=175$ and $K_{D}=4 \ldots \ldots$. . . 97

5.6 Comparison of the Performance of the Controllers in Simulation 100

5.7 Experimental Performance of the Controllers on the Real System 100

5.8 Comparison of Experimental and Simulated Performance . . . 105

5.9 Discussion of the Results . . . . . . . . . . . . . . 110

5.10 Further Research . . . . . . . . . . . . . . . . . 111

LIST OF REFERENCES . . . . . . . . . . . . . . . 113

\section{APPENDIX}

Visual Basic Code for PID-controller with Reset Windup . . . . 114

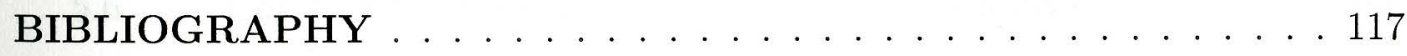




\section{LIST OF TABLES}

Table

Page

$2.1 \quad$ Parameters for the one-axis dynamic model . . . . . . . . . 23

2.2 Code to calculate nonlinear friction . . . . . . . . . . . 29

2.3 Set of friction parameter one-axis nonlinear model . . . . . . . 31

$3.1 \quad$ Parameters for the H-frame $20^{\text {th }}$-order model . . . . . . . . . . . 41

$4.1 \quad$ Parameters for the H-frame $8^{\text {th }}$-order model . . . . . . . . . 54

4.2 Set of friction parameter H-frame nonlinear model . . . . . . . . 59

5.1 Path deviations PID-control; $K_{p}=150, K_{I}=100$ and $K_{D}=4.81$

5.2 Poles \& Zeros for the SISO system controlled by PID-controller 188

5.3 Path deviations PID-control; $K_{p}=150, K_{I}=1500$ and $K_{D}=4 \quad 92$

5.4 Path deviations PD-control; $K_{p}=150$ and $K_{D}=4 \ldots$. . . . 97

5.5 Path deviations PD-control; $K_{P}=175$ and $K_{D}=4 \ldots . . .98$

5.6 Compared performances of the studied controllers . . . . . . . 100

5.7 Path deviations PID-control; $K_{P}=150, K_{I}=100$ and $K_{D}=4.102$

5.8 Path deviations PD-control; $K_{P}=175$ and $K_{D}=4 \ldots 102$

5.9 Path deviations PD-control increased gains Motor 2 . . . . . . 103 


\section{LIST OF FIGURES}

Figure

Page

$1.1 \quad$ General layout of the $\mathrm{H}$-frame positioning system . . . . . . . . 2

$1.2 \quad \mathrm{H}$-frame positioning system .............. . . 3

1.3 Relation rotational axis vs. linear axis .......... 7

$2.1 \quad$ One-Axis System . . . . . . . . . . . . . . 10

$2.2 \quad$ One-Axis System with coordinates and assumed belt behavior . 11

2.3 Timing belt as a serial connection of two springs . . . . . . 13

$2.4 \quad$ Block Diagram showing the open loop simulation setup . . . . . 15

2.5 One-Axis OL-test Motor Position . . . . . . . . . . . 16

2.6 One-Axis OL-test Motor Velocity . . . . . . . . . . . 16

$2.7 \quad$ One-Axis OL-test Motor Position different Stiffness . . . . . . . 17

2.8 One-Axis OL-test Motor Velocity different Stiffness . . . . . . . 18

2.9 One-Axis OL-test Motor Position different Stiffness . . . . . . 18

2.10 One-Axis OL-test Motor Velocity different Stiffness . . . . . . . 19

2.11 One-Axis OL-test Motor Position different Inertia . . . . . . . 19

2.12 One-Axis OL-test Motor Velocity different Inertia . . . . . . . . 20

2.13 One-Axis OL-test Motor Position different Friction . . . . . . . 21

2.14 One-Axis OL-test Motor Velocity different Friction . . . . . . . 21

2.15 One-Axis OL-test Motor Position best match 5V Input . . . . . 23

2.16 One-Axis OL-test Motor Velocity best match 5V Input . . . . . 24

2.17 Nonlinear friction, Stribeck effect, viscous and Coulomb friction 26

2.18 Nonlinear friction model, viscous and Coulomb friction . . . . . 27 
$2.19 \varphi_{1}$ axis nonlinear friction block diagram . . . . . . . . 28

2.20 nonlinear axis assembled to form the nonlinear plant model . . 30

2.21 nonlinear one-axis open loop simulation setup . . . . . . . 30

2.22 One-Axis OL-test Motor Position nonlinear Friction Model . . . 31

2.23 One-Axis OL-test Motor Velocity nonlinear Friction Model . . . 32

$3.1 \quad \mathrm{H}$-frame System . . . . . . . . . . . . . . . . 33

$3.2 \quad$ Generalized coordinates introduced to the H-frame System . . . 35

3.3 H-frame $20^{\text {th }}$-order model open loop simulation setup . . . . . 40

$3.4 \quad$ Open loop test y-motion motor angles . . . . . . . . . . 42

3.5 Open loop test y-motion end-effector position . . . . . . . . . 42

3.6 Open loop test $\mathrm{x}$-motion motor angles . . . . . . . . . . . 43

$3.7 \quad$ Open loop test $x-m o t i o n$ end-effector position . . . . . . . 43

4.1 H-frame with generalized coordinates for the simplified model . 49

$4.2 \quad$ Open loop test y-motion motor angles . . . . . . . . . . 55

4.3 Open loop test y-motion end-effector position . . . . . . . . 55

4.4 Open loop test $\mathrm{x}$-motion motor angles $\ldots \ldots \ldots 6$

$4.5 \quad$ Open loop test $\mathrm{x}$-motion end-effector position . . . . . . . 56

$4.6 \quad$ Nonlinear H-frame Simulink model . . . . . . . . . . . . . . . 59

4.7 Open loop test y-motion end-effector position; Input -6V/-6V . 60

4.8 Open loop test $\mathrm{y}$-motion motor angles; Input $-6 \mathrm{~V} /-6 \mathrm{~V} \ldots . . .60$

4.9 Open loop test x-motion end-effector position; Input $-6 \mathrm{~V} / 6 \mathrm{~V}$. . 61

4.10 Open loop test $\mathrm{x}$-motion motor angles; Input $-6 \mathrm{~V} / 6 \mathrm{~V}$. . . . . . 61

4.11 Open loop test $\mathrm{y}$-motion end-effector position; Input $-4 \mathrm{~V} /-4 \mathrm{~V} \quad$. 62 
4.12 Open loop test y-motion motor angles; Input $-4 \mathrm{~V} /-4 \mathrm{~V} \ldots \ldots 2$

4.13 Open loop test x-motion end-effector position; Input $-4 \mathrm{~V} / 4 \mathrm{~V}$. . 63

4.14 Open loop test $\mathrm{x}$-motion motor angles; Input $-4 \mathrm{~V} / 4 \mathrm{~V} \ldots \ldots 3$

4.15 Open loop test y-motion end-effector position; Input -2V/-2V . 64

4.16 Open loop test y-motion motor angles; Input $-2 \mathrm{~V} /-2 \mathrm{~V} \ldots \ldots 4$

4.17 Open loop test $\mathrm{x}$-motion end-effector position; Input $-2 \mathrm{~V} / 2 \mathrm{~V}$. . 65

4.18 Open loop test $\mathrm{x}$-motion motor angles; Input $-2 \mathrm{~V} / 2 \mathrm{~V} \ldots \ldots 5$

4.19 Change of Stiffness with respect to a change in $\mathrm{x} \ldots$. . . . . 69

4.20 Change of Stiffness with respect to a change in y . . . . . . 69

$5.1 \quad$ PID-control loop ....................... 72

5.2 PID-control of the $\mathrm{H}$-frame system . . . . . . . . 73

5.3 Trajectory for constant velocity profile . . . . . . . . 75

$5.4 \quad$ Velocity profile with constant acceleration and deceleration . . . 76

5.5 Trajectory with constant acceleration and deceleration . . . . 77

5.6 Test trajectory with constant acceleration and deceleration . . . 78

5.7 Reference inputs to the motors for square test trajectory . . . . 79

5.8 PID-controlled H-frame nonlinear model Simulink block diagram 79

5.9 End-effector path, PID-control $K_{P}=150, K_{I}=100$ and $K_{D}=481$

5.10 End-effector path for ideal rigid belt based on motor angles . . 83

5.11 Error from desired shape end-effector . . . . . . . . . . 84

5.12 Motor angles, PID-control $K_{P}=150, K_{I}=100$ and $K_{D}=4 \ldots 84$

5.13 Error motor angles, PID-control $K_{P}=150, K_{I}=100, K_{D}=4.85$

5.14 Effect of equal motor errors onto $x y$-position . . . . . . . 86 
$5.15 \quad$ Effect of unequal motor errors onto $x y$-position $\ldots . \ldots 87$

5.16 Motor angles, PID-control $K_{P}=150, K_{I}=1500$ and $K_{D}=4 \quad . \quad 90$

5.17 End-effector path, PID-control $K_{P}=150, K_{I}=1500, K_{D}=4 \quad .92$

5.18 Error integrals, $K_{P}=150, K_{I}=100$ and $K_{D}=4 \ldots . \ldots 94$

5.19 End-effector path, PD-control $K_{P}=150$ and $K_{D}=4 \ldots \ldots 6$

5.20 Error in shape end-effector, PD-control $K_{P}=150, K_{D}=4 \ldots 96$

5.21 Error motor angles, PD-control $K_{P}=150$ and $K_{D}=4 \ldots 97$

5.22 End-effector path, PD-control $K_{P}=175$ and $K_{D}=4 \ldots$. . . 98

5.23 Error in shape end-effector, PD-control $K_{P}=175, K_{D}=4 \ldots .99$

5.24 Error motor angles, PD-control $K_{P}=175$ and $K_{D}=4 \ldots 99$

5.25 End-effector path, PID-control $K_{P}=150, K_{I}=100$ and $K_{D}=4101$

5.26 End-effector path, PD-control $K_{P}=175$ and $K_{D}=4 \ldots 102$

5.27 Error motor angles, PD-control $K_{P}=175$ and $K_{D}=4 \ldots 103$

5.28 Error motor angles, PD-control increased Motor 2 gains . . . . 104

5.29 End-effector path, PD-control increased Motor 2 gains . . . . . 104

5.30 End-effector path simulated vs. experimental PID-case . . . . 105

5.31 End-effector path simulated vs. experimental PD-case . . . . 106

5.32 End-effector path simulated vs. experimental PD increased gains 107

5.33 Error from desired shape end-effector actual and encoder based 108

5.34 End-effector path simulated vs. experimental PD-case 2in square 109

5.35 End-effector path simulated vs. experimental PD-case $3 \frac{i n}{s} \ldots 109$ 


\section{CHAPTER 1}

\section{Introduction}

\subsection{The H-frame positioning system general setup}

The device to be studied is an XY-positioning system. The system is able to position a stage or so called end-effector in a horizontal plane. The end-effector can be equipped with a tool or some kind of other payload which needs to be positioned in $x$ and $y$. The system is called H-frame positioning system. This name refers to the layout of the system, which is shown in Figure 1.1 that looks like a capitalized H. There are two parallel tracks along which a bridge is lead through on linear ball bearing-blocks. On the bridge there is a third track mounted, perpendicular to the first two tracks, on which a cart slides. Those three tracks form a capitalized H. On each end of the two parallel tracks sits one pulley, where the ones at the lower end are directly attached to the motor shaft. On each end of the track on the bridge, there are two pulleys. The open timing belt is guided around those 8 pulleys. The open ends are both attached to the cart, which runs on the bridge. The system shows a parallel configuration, meaning that the actuator drive system is not an open kinematic chain. This parallel setup enables to have two stationary motors, which generates a couple of advantages in dynamic behavior, which is explained later. Another specialty of the system studied is the use of only one long timing belt, which wraps around the whole system including the motor pulley, to transform the rotational motion of the motor shafts into a linear $x$-motion of a bridge and a linear $y$-motion of a cart relative to the moving bridge. The overlapping of those two linear motions creates the $x y$-motion of the end-effector.

The motors used for the system are geared DC-brush type (gear ratio 5.9:1) and are equipped with encoders (321084 $\left.\frac{\text { counts }}{\text { rev }}\right)$ measuring the angular position of the motor shaft. Those motors are interfaced through amplifiers by a controlling 


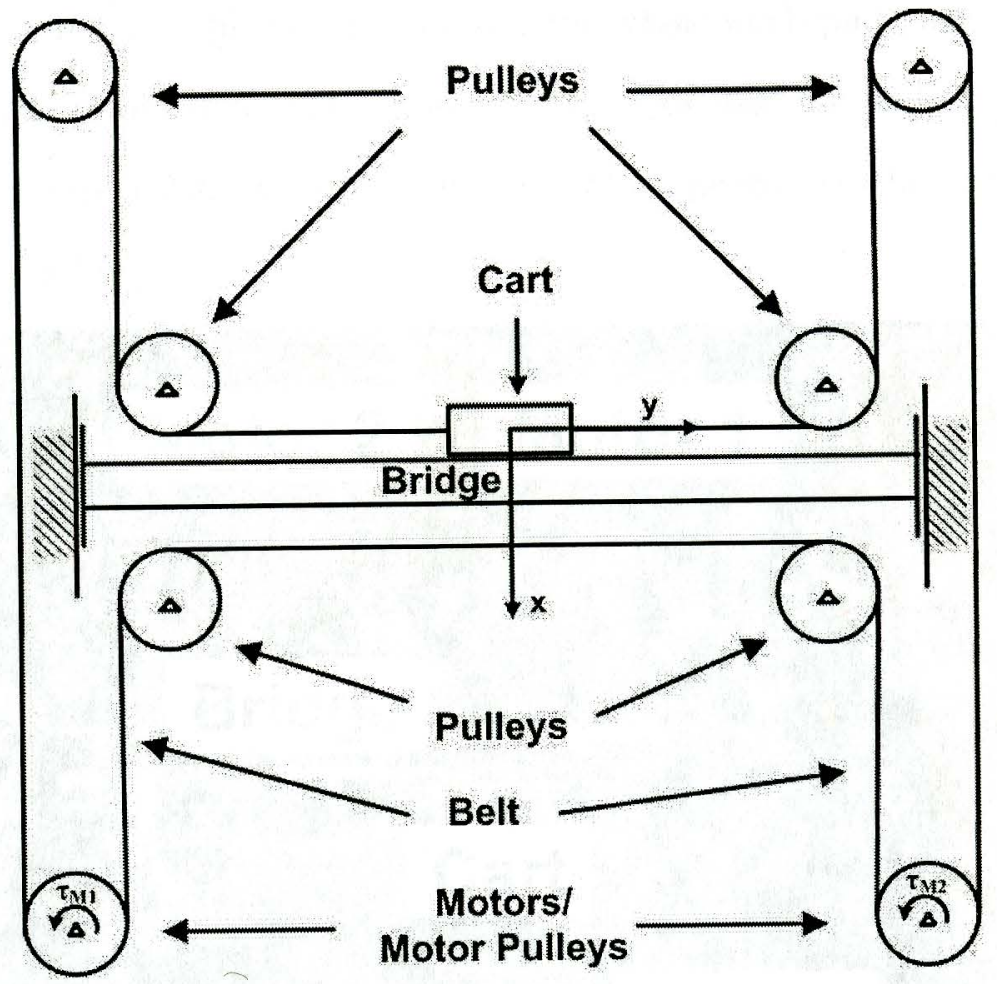

Figure 1.1. General layout of the H-frame positioning system

Personal Computer (PC). The PC interacts with the motors through 12-bit D/Aconverters and a 24-bit counter board. That means it can send input signals to each one of the motors, as well as reading out the encoder signals. The D/A- and A/Dconverters work at a sampling time $T_{\text {samp }}=0.001 \mathrm{~s}$ and have a input/output range $\pm 10 \mathrm{~V}$. The PC output Voltage is amplified by amplifiers before send to the motors as their input voltage. Furthermore the system has 4 limit switches placed at each end of the linear axis for safety reasons to shut down the motors in case of a runaway condition as well as for homing. Those Limit switches are interfaced by the PC through a parallel port. There are also two laser-displacement measuring devices (Model: optoNCDT; Manufacturer: MICRO-OPTRONIC Dresden) mounted onto the system that measure the $x$ - and $y$-position of the end-effector. Those lasers however, for reasons explained later as well as for their comparably small measuring 
range of about $10 \mathrm{~cm}^{2}$, which does not cover the whole workspace, are only used for verifying purposes of the actual end-effector position and not as feed back signals for control. Figure 1.2 shows a picture of the H-frame system the way it is set up in the Robotics Lab.

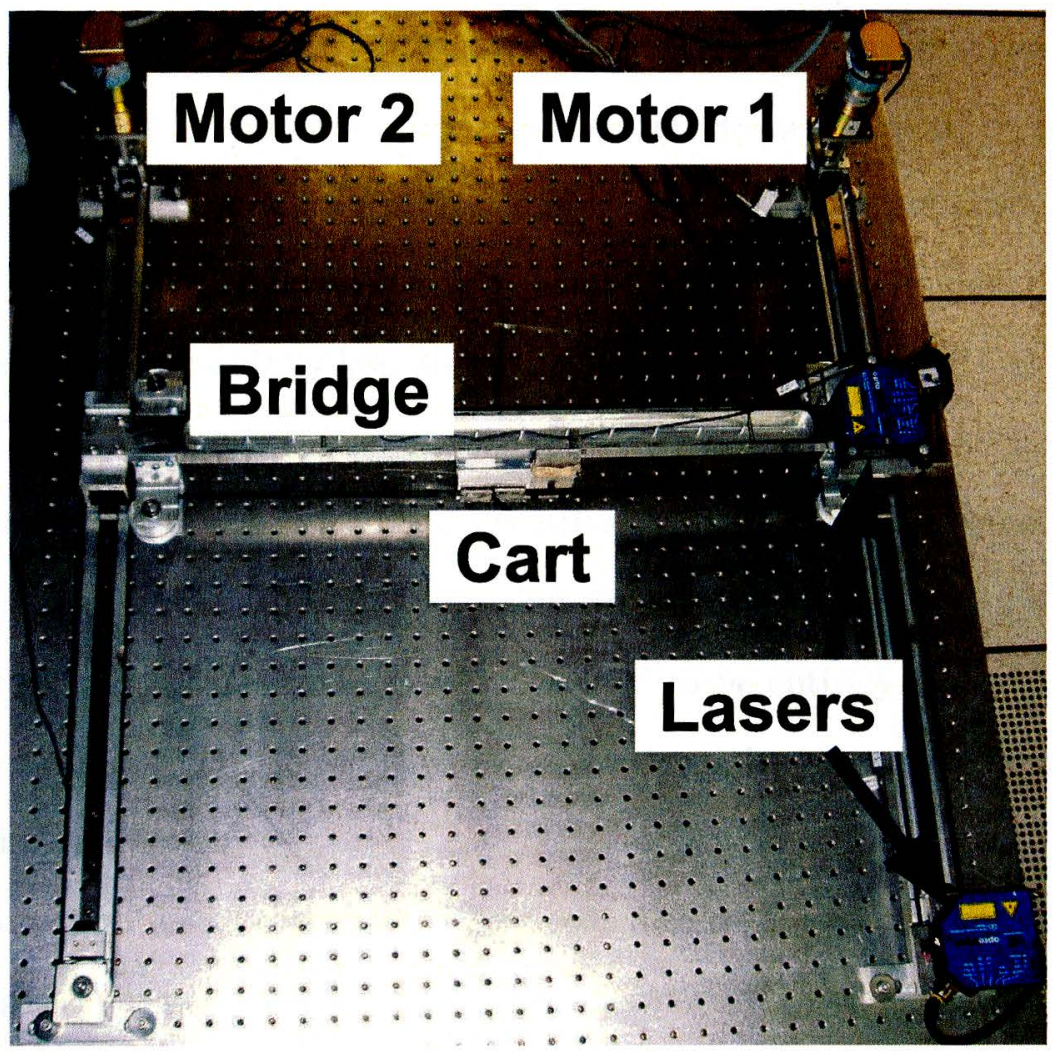

Figure 1.2. H-frame positioning system

\subsection{Motivations for the studying of the H-frame system}

The special layout of the H-frame system has certain advantages over nonparallel traditional XY-positioning tables. The parallel configuration with its stationary motors is very different from most commonly used serial stacked systems. Those stacked systems are built up from two independent linear positioning-axes. One of those axes, including the driving motor and all mechanical parts needed to transmit the rotational motion of the motor into a linear 
position of the stage, is then mounted perpendicular onto the other axis. The overlapping of the motion of those two independent linear axes gives then the position in $x$ and $y$. For arbitrary moition the top axis has to be moved by the bottom axis when that one moves, which results in a comparably big mass to be moved. The H-frame however with its parallel setup has comparably less masses to accelerate, since the motors are stationary and do not have to be moved and the mechanical parts to transmit the motor power to the end-effector are not heavy metal parts, like for example ball screws, but a relatively light belt. Those less masses to move result in less inertia and therefore in the ability to accelerate quicker and due to that a faster positioning with comparable motor power.

Another advantage of the $\mathrm{H}$-frame is a relatively low profile. Compared to stacked systems, which have to arrange their axis in two levels, in order to move one through the other, the H-frame can be designed to be entirely in one plane. This makes the H-frame ideal for applications, which might have some constraints in space in the $z$-direction.

The H-frame can also be seen as a low cost alternative to the traditional XYpositioning tables, since less precision parts are needed to transmit the rotational motion of the motors into a linear motion of the end-effector. Pulleys and timing belts are comparably cheap machine parts, which are standardized. Furthermore belt drives are known to be insensitive with regards to wheel alignment, which lowers costs for production and they are also comparably quiet power transmission systems.

However with those multiple advantages also comes the biggest disadvantage for the $\mathrm{H}$-frame system. This is that the timing belt is an elastic transmission element, compared to rigid elements like ball screws. This elasticity of the belt 
results in an uncertainty of exact end-effector position especially in non steady state modes like change in direction as well as due to nonlinear friction. This uncertainty results out of the fact, that the actual measured and controlled signals are the motor angles instead of the end-effector position. This is because a measurement system able to measure the position of the end-effector accurately over the whole workspace is not practical since it would be very expensive. To address this limitation, an adequate control strategy based on feedback of the motor angles will be investigated in this thesis. That is the reason why in this work a dynamic model will be developed and different control strategies will be explored to find a solution to solve this major disadvantage.

The solution for this problem is especially desirable since XY-positioning tables are widely used in industry and a device showing the same accuracy as traditional systems, but being able to position faster due to the advantages stated above, would increase productivity, by allowing more units being processed per unit of time.

The H-frame positioning system could be imagined for applications like laser cutting or welding, where the tool would be mounted on the end-effector, which cuts or welds sheets underneath the device. Also applying glue on a two dimensional part could be an application, in which the end-effector has to follow a trajectory closely. Pick and place tasks, for which the end-effector could be equipped with a gripper, is a rather point to point (PTP) task for the H-frame system. Less fitting however, due to the disadvantage of the flexible transmission link the belt, would be an application with major reaction forces like milling or drilling tasks. 


\subsection{Kinematics of the H-frame system}

The motion of the end-effector in $x$ - and $y$-direction is, as mentioned before, generated by an overlapping of the rotations of the two motors. This section will show the relationship between the rotations of the motors and $x y$-motion of the end-effector.

The linear axis are shown in Figure 1.1 and the positive rotation for each of the motors are defined as the mathematical positive rotation around the $z$-axis pointing outward from the paper plane. So that the motor torques shown in Figure 1.1 are positive torques.

Turning only one motor while keeping the other one still, results in a linear motion of the end-effector in a $45^{\circ}$ angle towards the $x y$-coordinate system. A positive rotation of motor 1 results in a motion in negative $x$ and negative $y$, while a negative rotation of the same motor would cause a motion in positive $x$ and $y$. Similarly a positive rotation of motor 2 while holding motor 1 still would cause a motion in positive $x$ - and negative $y$-direction and a negative rotation a linear motion of the end-effector in negative $x$ and negative $y$. Figure 1.3 shows the linearized rotational axis $\varphi_{1}$ and $\varphi_{2}$ which are the motor shaft angles of motor 1 and motor 2 respectively, as they lie towards the $x y$-coordinate system describing the end-effector position.

By multiplying the pulley radius $r$ with the motor angles, linearized rotations can now be expressed as vectors in the $\varphi_{1} r \varphi_{2} r$-coordinate system, and the $x y$-position of the end-effector can be seen by looking at the $x$ - and $y$-component of the vector sum of those $\varphi_{1} r$ - and $\varphi_{2} r$-vectors. A motion only in $x$-direction is being generated by rotating the motors by the same amount in opposite direction, while a motion only in $y$-direction is being generated by rotating both motors the same amount in the same direction. Through varying the amounts of rotation for each motor a 


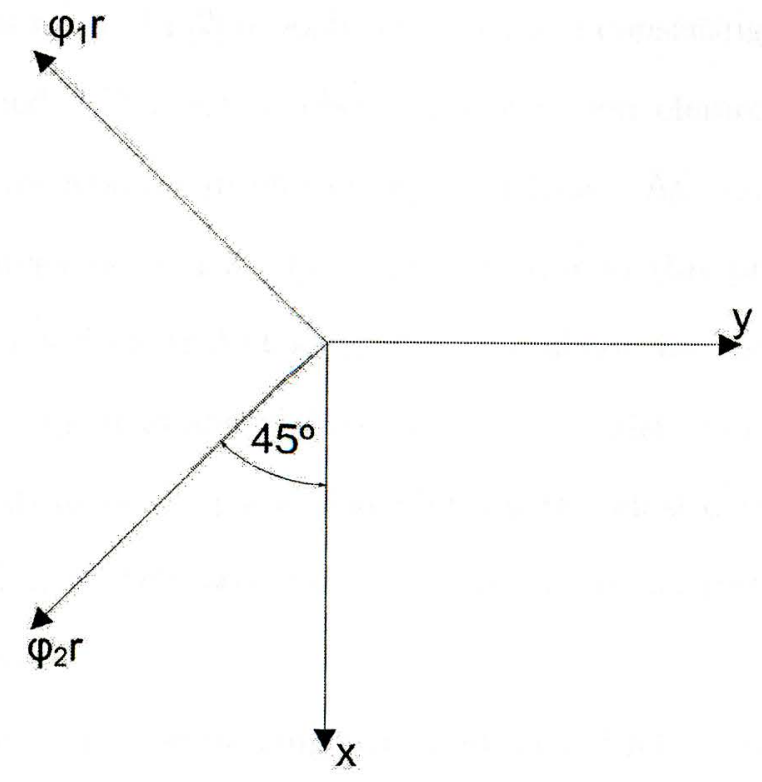

Figure 1.3. Relation rotational axis vs. linear axis

motion in any angle towards the $x$-axis can be reached.

This relation can be expressed in a matrix equation shown in equation 1.1.

$$
\left[\begin{array}{l}
\Delta x \\
\Delta y
\end{array}\right]=\left[\begin{array}{cc}
-\frac{1}{2} r & \frac{1}{2} r \\
-\frac{1}{2} r & -\frac{1}{2} r
\end{array}\right]\left[\begin{array}{c}
\Delta \varphi_{1} \\
\Delta \varphi_{2}
\end{array}\right]
$$

\subsection{Literature Review}

Survey of literature was performed related to previous work on control of positioning systems with flexible transmission elements. There was no publications found describing a similar parallel $x y$-positioning system as the studied $\mathrm{H}$ frame system, which works with stationary motors and one timing belt. Most $x y$-positioning systems described were stacked ball screw systems or stacked belt drive systems.

One publication was found [1] that describes the control of a stacked ball screw $x y$-positioning system, but addresses the problem that ball screws as well have a finite stiffness and therefore lead to a difference between end-effector position and motor position. A torsional displacement feedback control was proposed to improve 
the tracking in this work. In [2] a positioning system consisting of two stacked belt drive axis is studied. The use of elastic transmission elements (the belts) lead to the described uncertainty in end-effector position. An observer based sliding mode control strategy is used in that work to address this problem. Both those very different proposed control strategies in the above mentioned works have in common, that an accurate model of the system has to exist to be able to successfully implement those strategies. They also identify the elastic transmission as well as nonlinear friction as the two major challenges in accurately controlling $x y$ positioning systems.

Further literature review dealing with nonlinear friction and elastic transmission elements for only one axis systems has also been carried out. Methods proposed to control one axis belt drive systems found in the literature are fuzzy-logic [3], sliding mode methods combined with Lyapunov design [4] and feed-forward compensator under maximum acceleration and velocity constraints [5]. References [6] and [7] propose adaptive control strategies to address the problems caused by nonlinear friction. Again for the majority of those control strategies proposed an accurate model of the system to be controlled has to be accessible.

From the above we can see that nonlinear friction as well as the flexibility of the transmission element plays a major role in choosing an appropriate control strategy. It can also be seen, that for most control strategies proposed in the literature to handle those challenges an accurate model description of the system dynamics is needed.

\subsection{Scope of the Thesis}

In order to accurately control the $\mathrm{H}$-frame system the dynamics including the nonlinearities have to be understood. Also most sophisticated control strategies, as the literature review revealed, require an accurate model of the dynamics of the 
system to be controlled. This is why in this work, first a simple one-axis system is studied to understand the effects related to belt drive positioning systems. For this system a linear as well as a nonlinear model is being derived, simulated and verified by comparing it with experimental data.

The findings from those studies are utilized to derive first a linear model of the $\mathrm{H}$-frame system. A $20^{\text {th }}$-order model was derived using Lagrange approach. The model is then simulated and verified with experimental results from the real system. To simplify computational efforts, it is examined if the $20^{\text {th }}$-order linear system can be simplified to a lower order system. Assumptions are made and a new $8^{\text {th }}$-order model obtained and verified by comparing it to the open loop simulation results of the $20^{\text {th }}$-order model as well as to the experimental data. Based on this simplified model a nonlinear model of the $\mathrm{H}$-frame system is derived and verified.

Finally the performance of basic controllers to control the $\mathrm{H}$-frame system are examined both in simulation utilizing the obtained nonlinear model and on the real system. Specifics of the H-frame system that have to be considered in order to control it accurately are pointed out in that process. Also the effects of challenges, like the uncertainty of end-effector position due to an elastic transmission between the controlled motors and the end-effector as well as the presence of nonlinear friction, onto accurate control of the system are discussed. 


\section{CHAPTER 2}

\section{The One-Axis Model}

In order to familiarize with the dynamic characteristics of a belt driven positioning system as well as to verify assumptions that where being made concerning for example the friction and the stiffness of the belt, a 1-axis positioning system as shown in Figure 2.1 was studied first.

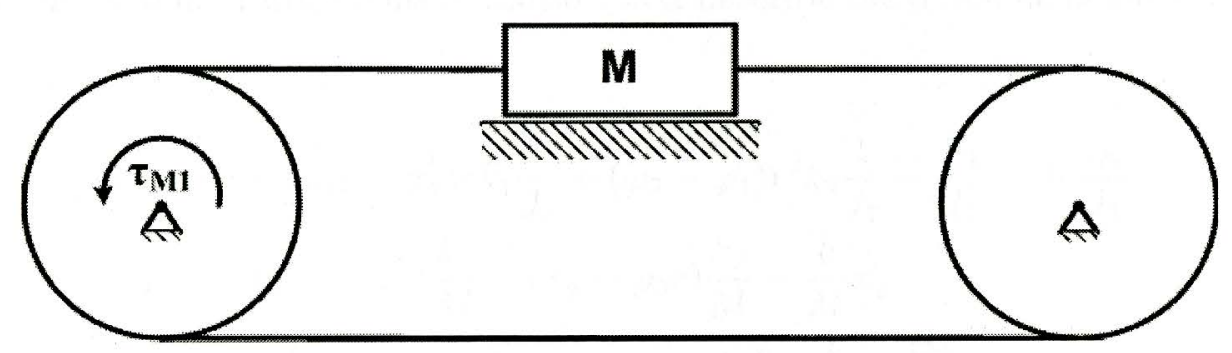

Figure 2.1. One-Axis System

\subsection{Equations of Motion}

In order to acquire the equations of motion describing the dynamic behavior of such a system, the timing belt was assumed to have the behavior of linear springs with a stiffness $k_{i} ; i=1,2,3$ as shown in Figure 2.2, also linear friction was assumed. So that the Forces of the timing belt onto the cart as well as on the pulleys are of the form $F_{t b}=k * \Delta l$, the friction force acting on the car is of the form $F_{f r}=b * v_{r e l}$ and the friction torques acting on the pulleys are of the form $\tau_{f r}=B * \omega_{r e l}$. Where $\Delta l, B, b, v_{r e l}$ and $\omega_{r e l}$ are the difference in belt length, rotational and the linear friction coefficient and the linear relative velocity as well as the relative angular velocity respectively. 


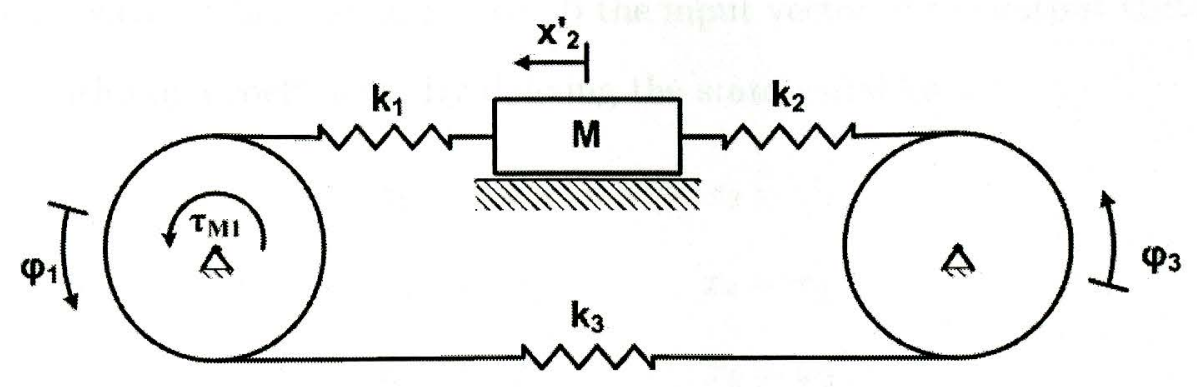

Figure 2.2. One-Axis System with coordinates and assumed belt behavior

Through the application of Newton's Laws of motion, it was easy to find the following system of Equations of Motion that describe the dynamic behavior of the system.

$$
\begin{aligned}
\ddot{\varphi}_{1} & =-\left(\varphi_{1} r-x_{2}^{\prime}\right) r k_{1} \frac{1}{J_{1}}+\left(\varphi_{3}-\varphi_{1}\right) r^{2} k_{3} \frac{1}{J_{1}}-\frac{B_{1}}{J_{1}} \dot{\varphi_{1}}+\frac{\tau_{M}}{J_{1}} \\
\ddot{x_{2}^{\prime}} & =\left(\varphi_{1} r-x_{2}^{\prime}\right) \frac{k_{1}}{M}-\left(x_{2}^{\prime}-\varphi_{3} r\right) \frac{k_{2}}{M}-\frac{b}{M} \dot{x_{2}^{\prime}} \\
\ddot{\varphi_{3}} & =\left(x_{2}^{\prime}-\varphi_{3} r\right) r k_{2} \frac{1}{J_{2}}-\left(\varphi_{3}-\varphi_{1}\right) r^{2} k_{3} \frac{1}{J_{2}}-\frac{B_{2}}{J_{2}} \dot{\varphi_{3}}
\end{aligned}
$$

Where $\varphi_{1}, \varphi_{2}$ and $x_{2}^{\prime}$ are rotational and linear coordinates as shown in Figure 2.2, $J_{i}(i=1,2)$ is the inertia of the pulleys, $M$ is the mass of the cart, $b$ and $B_{i}$ $(i=1,2)$ are friction coefficients, $r$ the radius of the pulleys and $\tau_{M}$ is the Motor Torque applied, which is given by

$$
\tau_{M}=\frac{K_{t}}{R} V_{i n}-\frac{K_{t} K_{e}}{R} \dot{\varphi}_{1}
$$

Where $V$ in is the input voltage given to the Motor and therefore the input signal to the one-axis belt drive system. $K_{t}, K_{e}$ and $R$ are the torque constant, the back-emf constant and the resistance of the motor respectively.

In order to easily simulate the system in Simulink the model now was transferred into State Space form[8].

$$
\begin{aligned}
& \dot{\mathbf{x}}=\mathbf{A x}+\mathbf{b} u \\
& y=\mathbf{c x}+d u
\end{aligned}
$$


Where $\mathbf{A}$ is called the system matrix, $\mathbf{b}$ the input vector, $\mathbf{c}$ the output vector and $d$ the feedthrough coefficient. By defining the state variables as:

$$
\begin{array}{ll}
x_{1}=\varphi_{1} & x_{2}=\dot{\varphi_{1}} \\
x_{3}=x_{2}^{\prime} & x_{4}=\dot{x}_{2}^{\prime} \\
x_{5}=\varphi_{3} & x_{6}=\dot{\varphi_{3}}
\end{array}
$$

We get the following expressions for the matrices.

System Matrix

$$
\mathbf{A}=\left[\begin{array}{cccccc}
0 & 1 & 0 & 0 & 0 & 0 \\
-\frac{\left(k_{1}+k_{3}\right) r^{2}}{J_{1}} & -\frac{B_{1}}{J_{1}}-\frac{K_{t} K_{e}}{R J_{1}} & k_{1} \frac{r^{2}}{J_{1}} & 0 & k_{3} \frac{r^{2}}{J_{1}} & 0 \\
0 & 0 & 0 & 1 & 0 & 0 \\
\frac{k_{1}}{M} & 0 & -\frac{k_{1}+k_{2}}{M} & \frac{b}{M} & \frac{k_{2}}{M} & 0 \\
0 & 0 & 0 & 0 & 0 & 1 \\
k_{3} \frac{r^{2}}{J_{2}} & 0 & k_{2} \frac{r^{2}}{J_{2}} & 0 & -\frac{\left(k_{2}+k_{3}\right) r^{2}}{J_{2}} & -\frac{B_{2}}{J_{2}}
\end{array}\right]
$$

Input Vector

$$
\mathbf{b}=\left[\begin{array}{c}
0 \\
\frac{K_{t}}{R J_{1}} \\
0 \\
0 \\
0 \\
0
\end{array}\right]
$$

Output Vector

$$
\mathbf{c}=\left[\begin{array}{llllll}
1 & 0 & 0 & 0 & 0 & 0
\end{array}\right]
$$

Feedthrough coefficient

$$
d=0
$$

\subsection{Determination of the Parameters}

In order to reflect the dynamic behavior of the system accurately the parameters of friction, inertia and mass as well as stiffness have to be determined. After finding values for those parameters, the accuracy of the model has to be verified by experimental data, since not all of the parameters can be determined as accurately and easily as for example the mass of the cart. 


\subsubsection{Stiffness}

The stiffness of the belt sections were assumed to be of the form

$$
k=\frac{A E}{l}
$$

where $A$ is the cross-section-area of the Belt $E$ is the E-Modulus and $l$ the length of the belt section.

Since a timing belt consists of teeth and belt regions without teeth the crosssection cannot be chosen trivially. However if the regions with and without teeth are being considered as a serial connection of two springs as shown in Figure 2.3, then it can be seen that as a good assumption the cross-section can be chosen to be the one of the region without teeth.

This is because the equation for the effective stiffness of serial springs is.

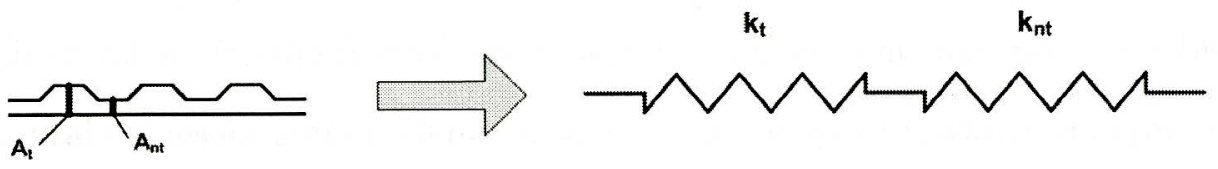

Figure 2.3. Timing belt as a serial connection of two springs

$$
k_{e f f}=\frac{k_{t} k_{n t}}{k_{t}+k_{n t}}
$$

Therefore since $A_{t}>>A_{n t}$ and also $k_{t}>>k_{n t}$, this leads to the transformation of equation 2.12 to

$$
k_{e f f} \approx k_{n t}=\frac{A_{n t} E}{l}
$$

\subsubsection{Inertia \& Mass}

For the derivation of the dynamic model the mass of the belt is assumed to be negligible compared to the inertia of the pulley as well as to the mass of the cart. So that the mass of the cart and the inertia of the pulleys and not the mass of the belt are effecting the dynamic behavior of the system. 
While the mass of the cart can be easily and precisely determined by disassembling and scaling it, the inertia of the pulleys could only be approximated. The approximation is based on the calculations obtained from a Solid Works model of the pulley, which has slight uncertainties since the exact aluminium alloy is not known. Also the geometry of the Solid Works model marginally differs from those pulleys used in the system.

\subsubsection{Friction}

The friction of the system is assumed to be viscous friction, which is proportional to a friction coefficient $b$ ( $B$ for the friction torques of the pulleys). The value of the coefficient is affected by different effects, the configuration of the surfaces rubbing against each other is one example for those effects, the lubrication of the ball bearings of the pulleys another.

Since values for the friction coefficient for this system were not available, experimental values for similar elements as used in the system, which were previously determined in the Robotics Lab, where used for the dynamic model. However it should be noted, that those friction values are probably the least accurate from the above due to this approximation.

\subsection{Simulation of the One-Axis model}

To verify the dynamic model as well as the assumptions made regarding the three stiffness coefficients, inertia and friction the dynamic model was simulated in Simulink and compared to experimental data gained from the real system. Therefore open loop tests where run on the real system as well as open loop simulations with previously obtained model. To compare the open loop response of the dynamic model and the real system a 0.25 seconds impulse of different voltages $(10 \mathrm{~V}$, $5 \mathrm{~V}$ ) was imputed into the system and the angle position (converted into meters 
by multiplying with the pulley radius) and the velocity of the motor pulley were recorded.

In the real system the data was recorded by a data collection software programmed in Visual Basic that utilized the encoder measurements of the motor. For the dynamic system the A-Matrix as well as the $\mathbf{b}$-, and $\mathbf{c}$-Vectors of the state space model where computed with the above chosen parameters in Matlab and then simulated in Simulink utilizing the block diagram shown in Figure 2.4. The c-Matrix in this case is a $2 \times 6$ Matrix of the form

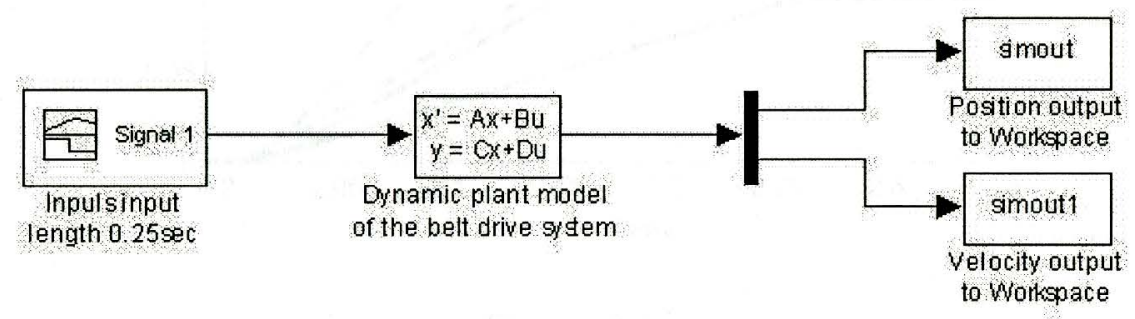

Figure 2.4. Block Diagram showing the open loop simulation setup

$$
\mathbf{c}=\left[\begin{array}{llllll}
1 & 0 & 0 & 0 & 0 & 0 \\
0 & 1 & 0 & 0 & 0 & 0
\end{array}\right]
$$

which computes an $2 \times 1$ output vector containing the motor angle and the motor velocity. This vector is then split up in its elements and stored in the workspace for plotting purposes.

The simulation has been carried out for $5 \mathrm{~V}$ and $10 \mathrm{~V}$ input voltages and then plotted in comparison to the data acquired by the testing in the real system. This gave the plots shown in Figure 2.5 and 2.6.

In those plots it can be seen, that the response of the simulated system does not match the experimental system very well. Even though the dynamics seem to be captured well, the response of the simulated system is less than what was observed in the real system. However since the dynamics seem to match in general 


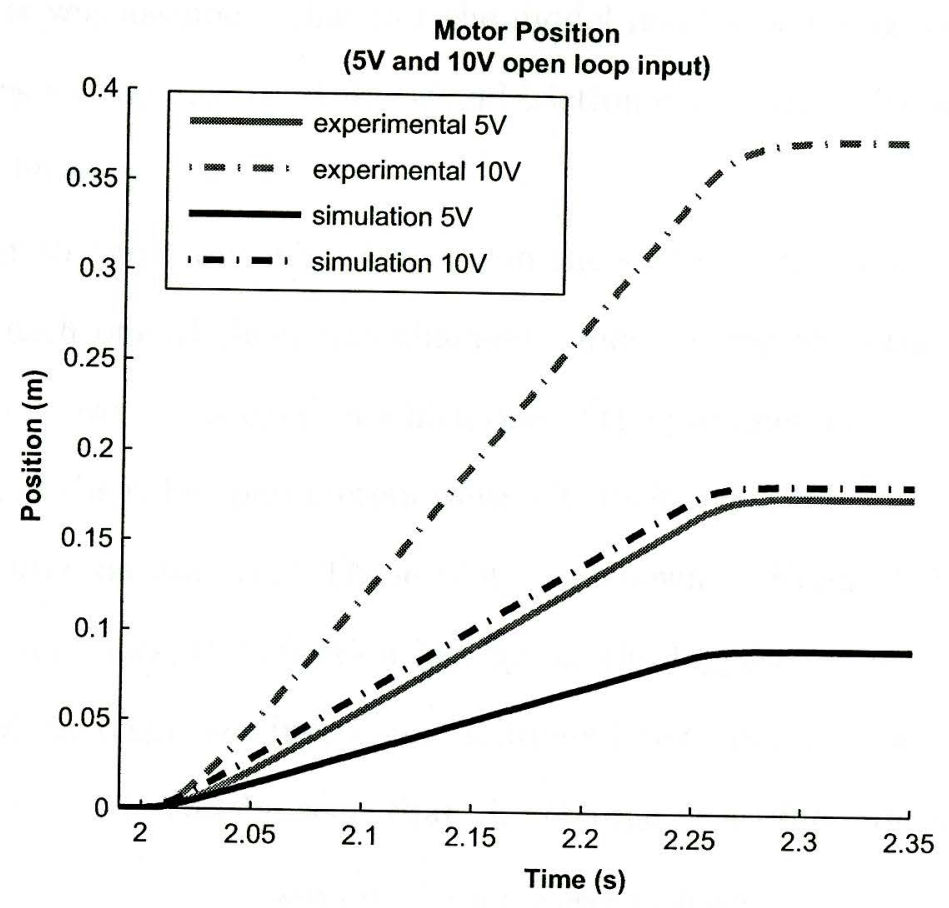

Figure 2.5.

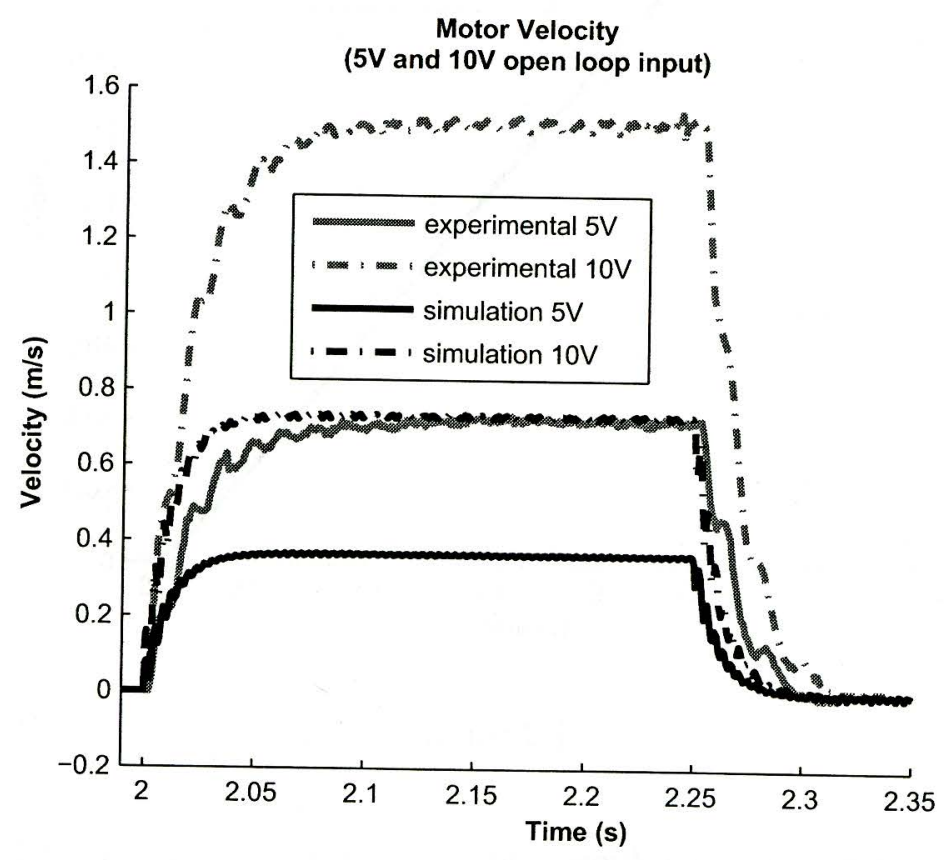

Figure 2.6. 
pretty well it was assumed, that not the model itself was wrong, but the approximated values for the inertia, stiffness and friction coefficient parameters where not accurately chosen.

In order to figure out what impact on the system either one of those parameters have, each one of them was changed while keeping the other two constant. Therefore plots were produced in which one of the parameters was doubled as well as halved, and the other parameters were left unchanged. This was made for each one of the three parameters. Those plots are shown in Figure 2.7-2.14.It can be seen from those plots, that friction has by far the biggest influence regarding the magnitude of the response. Inertia and stiffness have minor effects on the dynamics of the system. It can be seen that the change in stiffness does not apprecia-

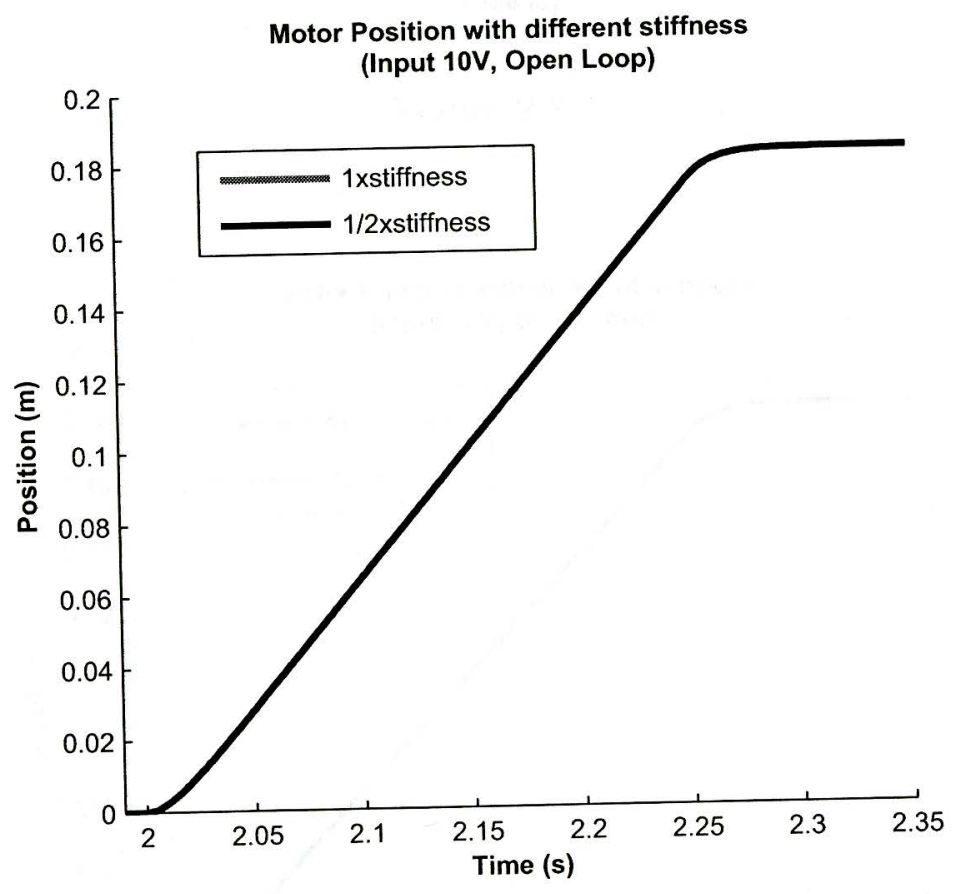

Figure 2.7.

bly change the position response. Only the oscillation seen in the velocity profile changes marginally when changing the stiffness factor by 2 . Since the inertia 


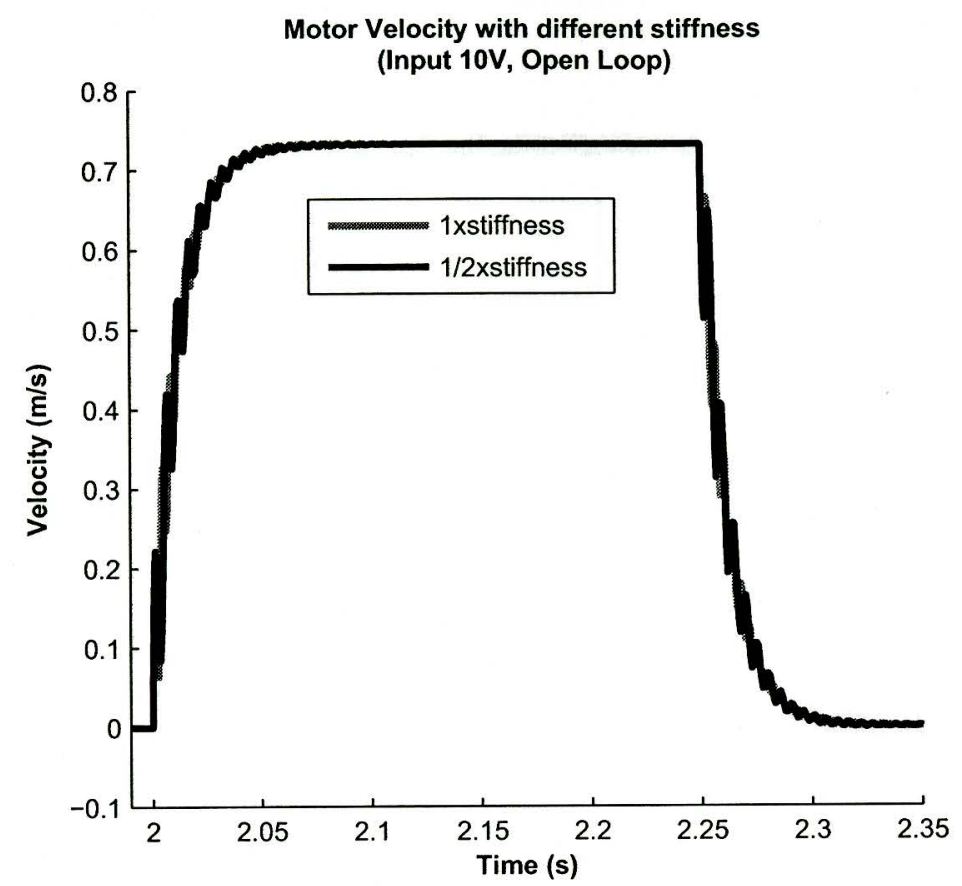

Figure 2.8.

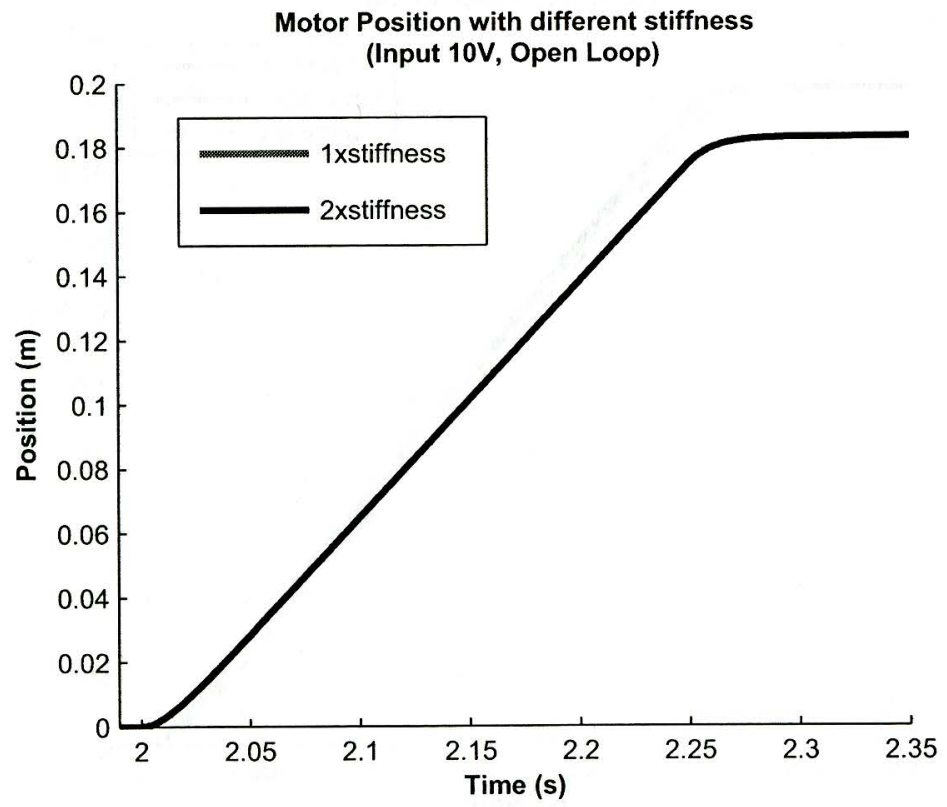

Figure 2.9. 


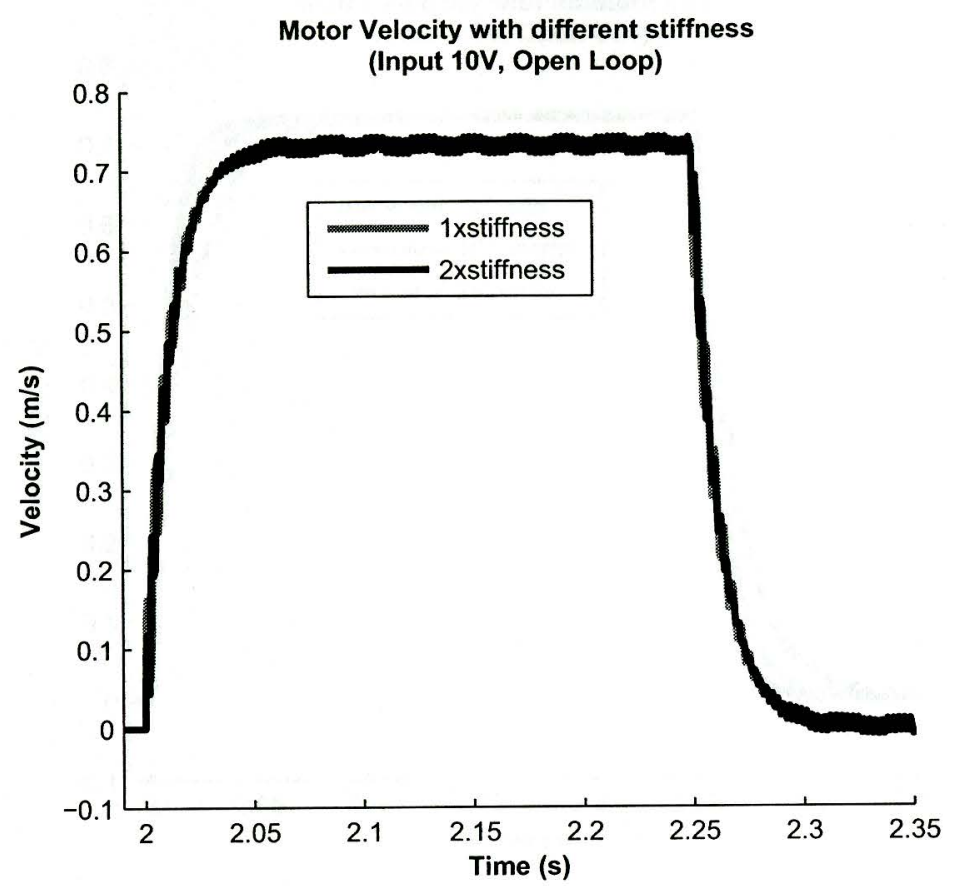

Figure 2.10.

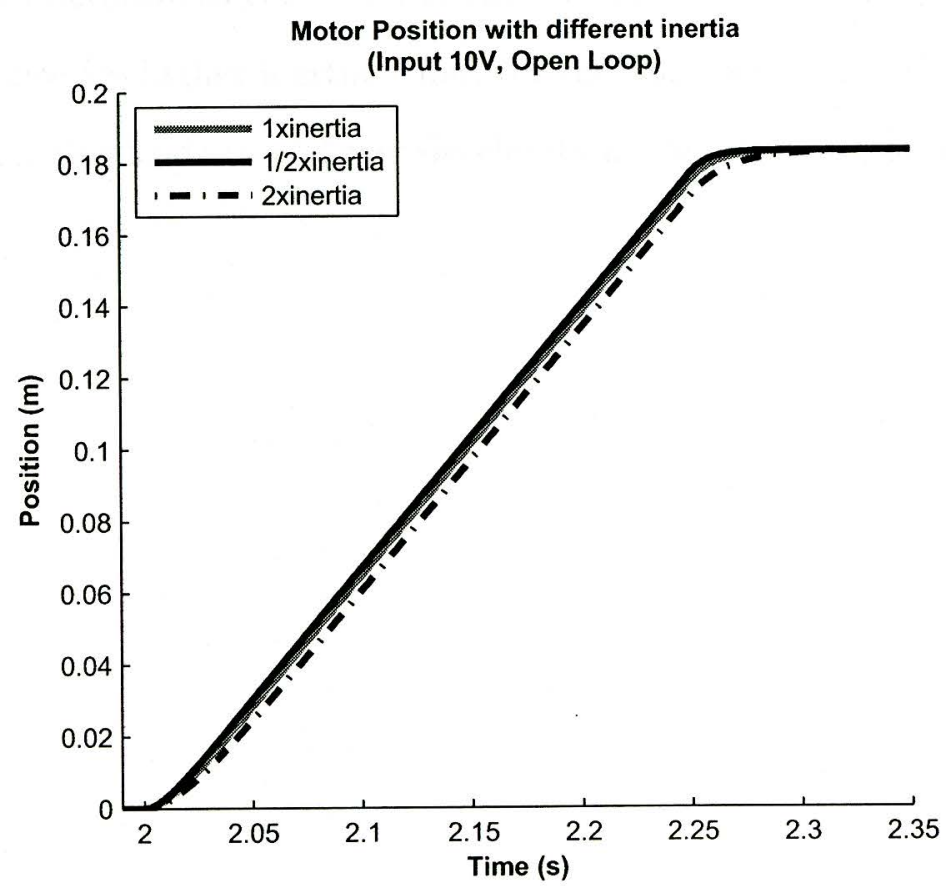

Figure 2.11. 


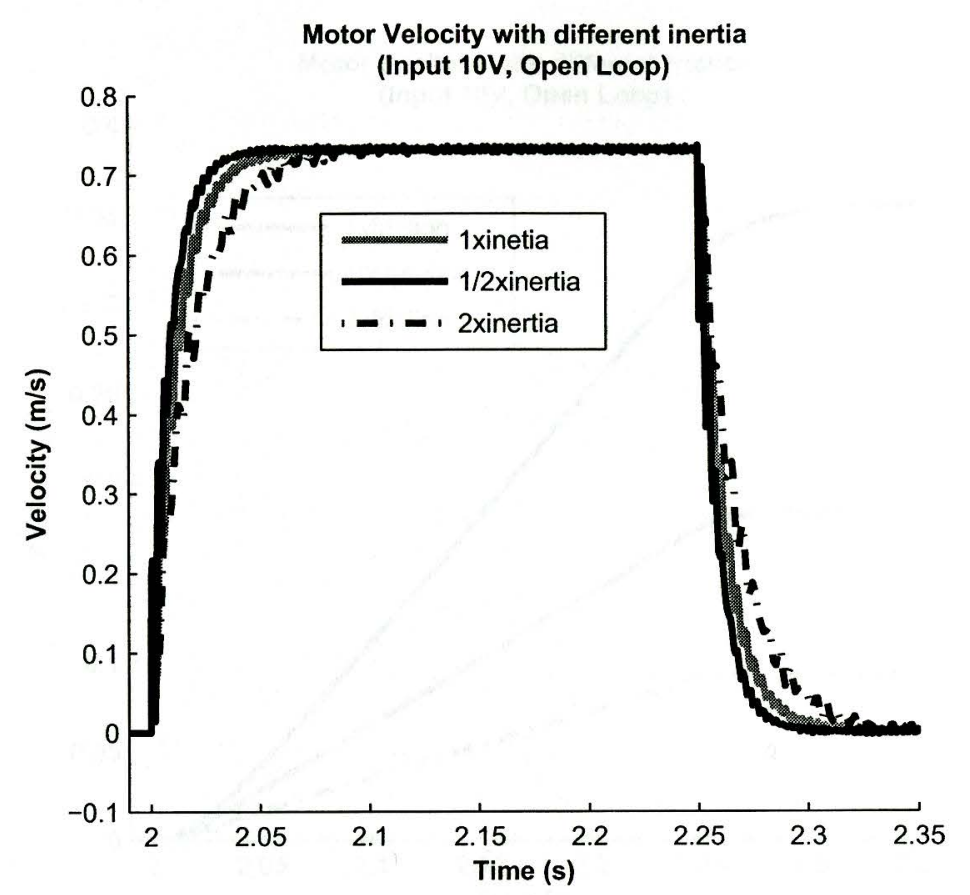

Figure 2.12.

of the system determines the speed of the response, the plots show as expected a slower response for higher inertia. However the final position is the same, since a higher inertia also leads to a slower deceleration after shutting off the motor. 


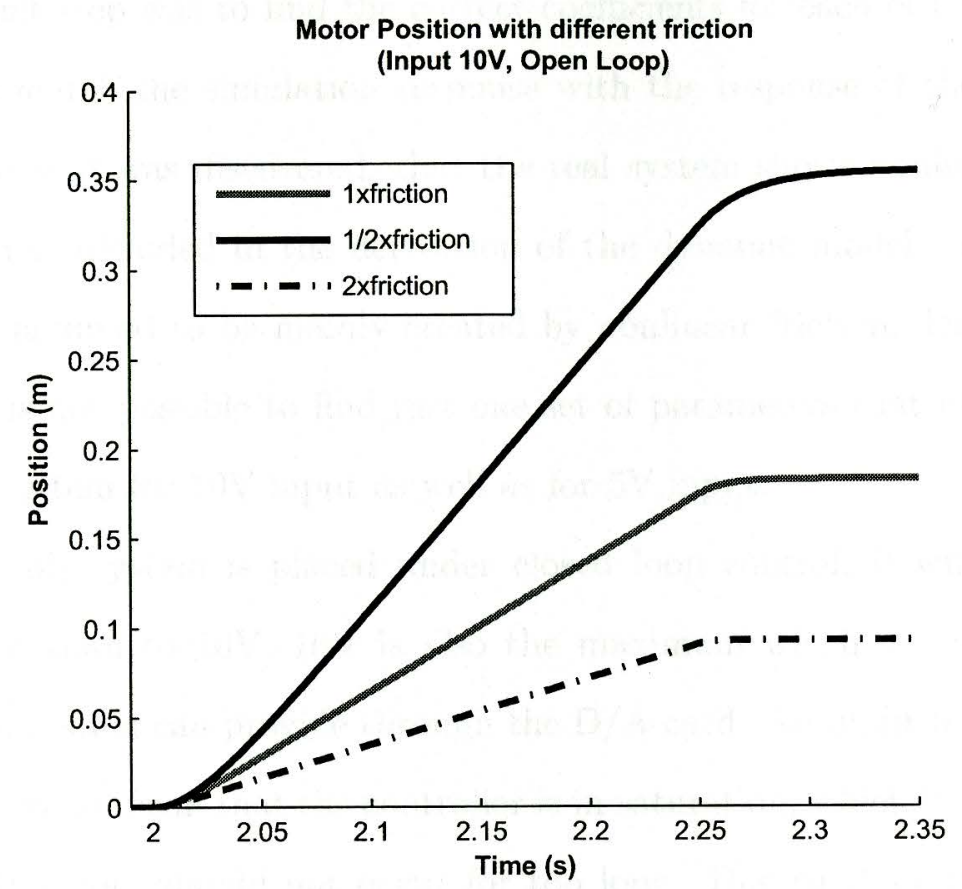

Figure 2.13.

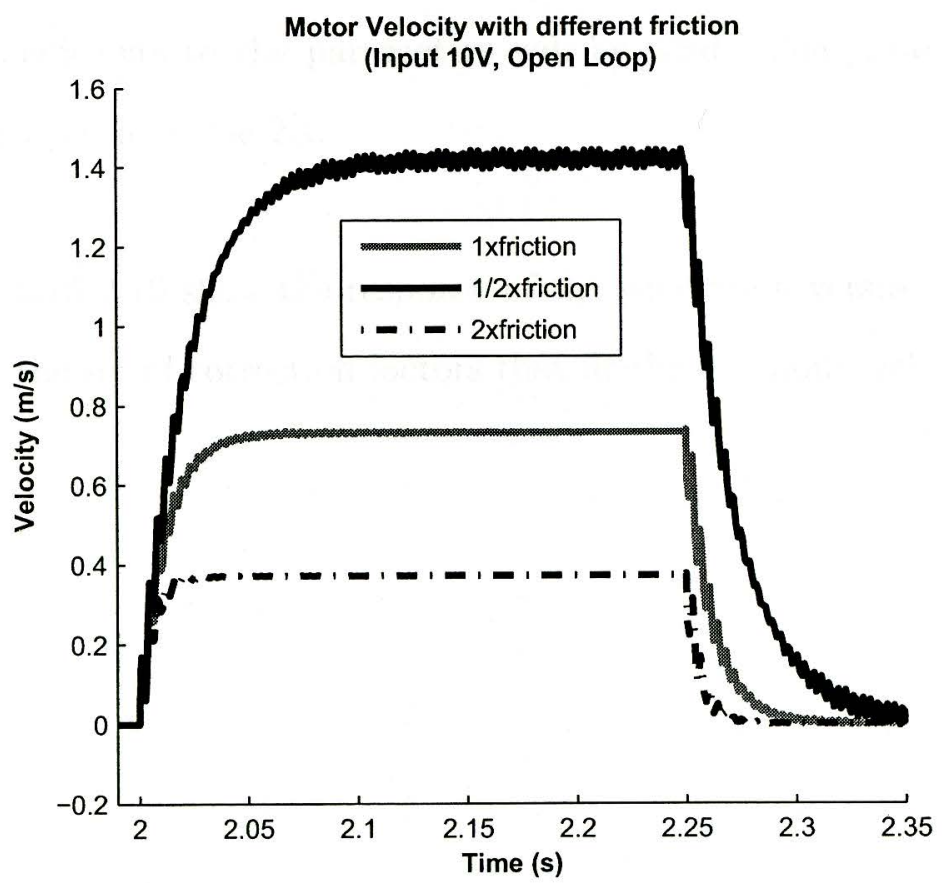

Figure 2.14. 
The next step was to find the correct coefficients for each of the parameters, in order to match the simulation response with the response of the real system. In this process it was discovered, that the real system shows nonlinear behavior, which was not included in the derivation of the dynamic model. This nonlinear behavior is assumed to be mainly created by nonlinear friction. Due to the nonlinearity it is not possible to find just one set of parameters that fit the response of the real system for $10 \mathrm{~V}$ input as well as for $5 \mathrm{~V}$ input.

When this system is placed under closed loop control, it will be operated closer to $5 \mathrm{~V}$ than to $10 \mathrm{~V}$. $10 \mathrm{~V}$ is also the maximum which the computer that controls the system can provide through the D/A-card. An input to the system of about $10 \mathrm{~V}$ would mean that the controller is in saturation, which is an undesirable state and therefore should not occur for too long. Due to those two reasons, it was decided, that a good match of the characteristics around $5 \mathrm{~V}$ is more desirable than for $10 \mathrm{~V}$. Therefore for further steps which require a linear model, the $5 \mathrm{~V}$ correction coefficients to the parameters will be used. The parameters for that case are displayed in Table 2.1.

Figures 2.15-2.16 show the response of the simulation versus the real system response for the set of correction factors that fit the $5 \mathrm{~V}$ input well. 


\begin{tabular}{|c|c|c|}
\hline & Variable & Value \\
\hline \multirow{2}{*}{ Radius } & $r$ & $0.0194 \mathrm{~m}$ \\
\hline \multirow{2}{*}{ Stiffness } & $k_{1}$ & $1.3276 \times 10^{5} \frac{\mathrm{N}}{\mathrm{m}}$ \\
\cline { 2 - 3 } & $k_{2}$ & $1.3276 \times 10^{5} \frac{\mathrm{N}}{\mathrm{m}}$ \\
\cline { 2 - 3 } & $k_{3}$ & $5.8885 \times 10^{4} \frac{\mathrm{N}}{\mathrm{m}}$ \\
\hline \multirow{2}{*}{ Friction } & $B$ & $0.0021 \mathrm{Nms}$ \\
\cline { 2 - 3 } Inertia \& Mass & $b$ & $20.3259 \frac{\mathrm{Ns}}{\mathrm{m}}$ \\
\cline { 2 - 3 } & $\mathrm{J}$ & $6.3 \times 10^{-5} \mathrm{Kg} \mathrm{m}^{2}$ \\
\hline \multirow{3}{*}{ Motor constants } & $K_{t}$ & $0.362 \mathrm{Kg}$ \\
\cline { 2 - 3 } & $K_{e}$ & $23 \times 10^{-3} \frac{\mathrm{Nm}}{\mathrm{A}}$ \\
\cline { 2 - 3 } & $R$ & $0.71 \Omega$ \\
\hline
\end{tabular}

Table 2.1. Parameters for the one-axis dynamic model

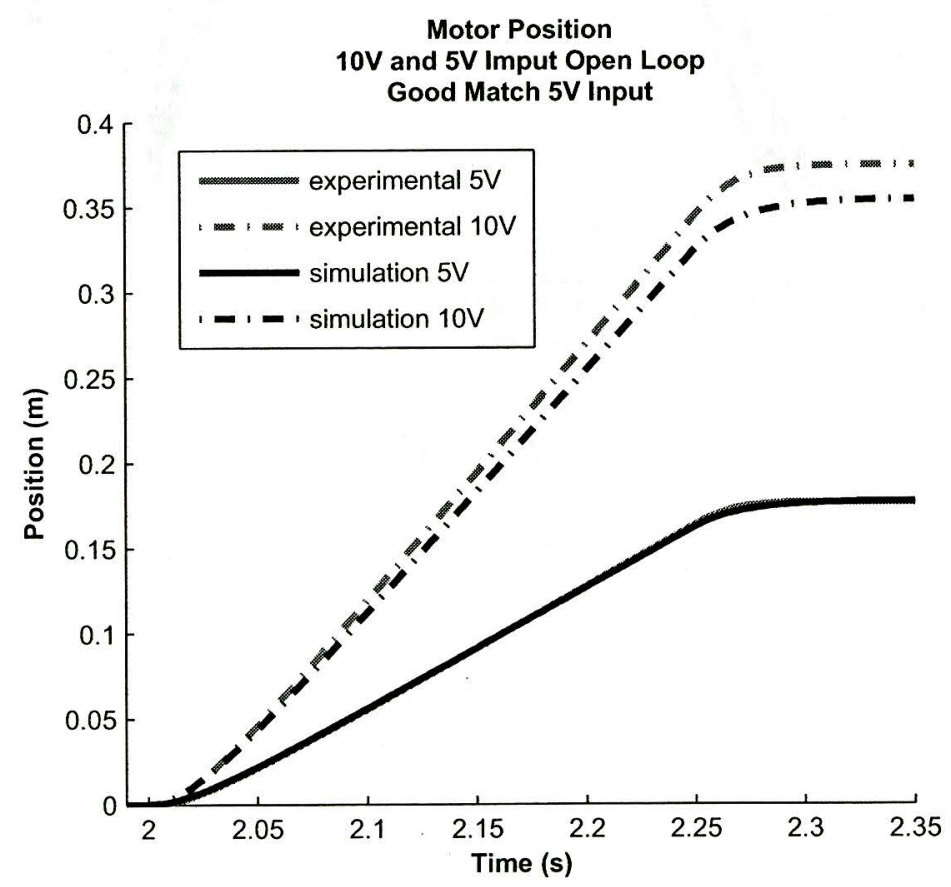

Figure 2.15. 


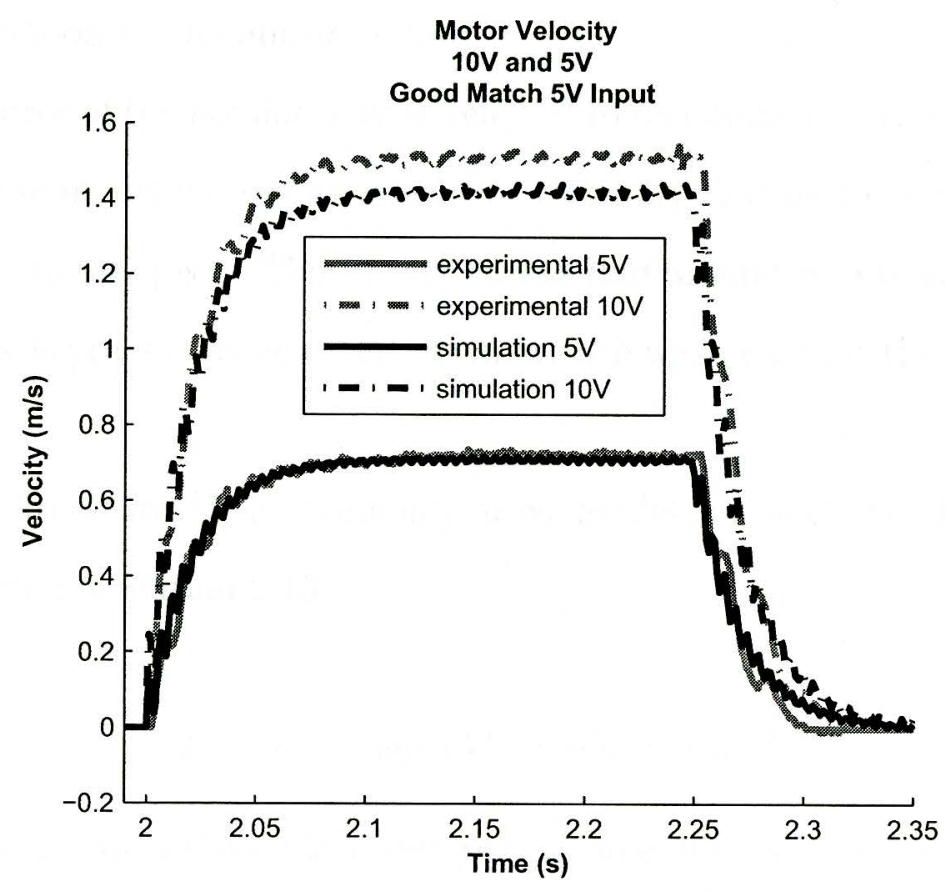

Figure 2.16. 


\subsection{Nonlinear Model of the One-Axis System}

As shown above the linear model can accurately reflect the response to only one certain input voltage. In control applications however the input voltage is not necessarily well predictable and most certainly not constant, since in closed loop control the input voltage to the plant is error driven. Therefore it is desirable to come up with a nonlinear simulation model, which reflects the nonlinearities and therefore can give a better idea if the chosen linear control strategies are robust enough to run on the nonlinear system.

The source of the nonlinearity is believed to be nonlinear friction. Initially the friction in the modeling was assumed to be strictly viscous friction and therefore proportional to the speed. This in turn means that around zero velocity the friction force/torque is practically zero. From experience we know that this however is not the case.

In the literature [7] a commonly used model for nonlinear friction can be found, shown in equation 2.15 .

$$
F_{f}=b v+\operatorname{sign}(v) F_{c}+\operatorname{sign}(v) F_{s} e^{-F_{\tau} v^{2}}
$$

The model consists of three terms describing three different friction related effects. The first term describes the so called viscous friction, which is what was assumed so far. The second term accounts for the Coulomb friction, which is an offset value depending on the direction of motion. This effect accounts for the fact that for velocities close to zero the friction force is not zero. The third and last term is called Stribeck effect. It is based on the observation that the friction drops a certain amount after motion has started. $F_{f}, F_{c}$ and $F_{s}$ in the equation are called the friction force, the Coulomb force and the static force respectively, $F_{\tau}$ is the Stribeck related positive parameter and $b$ the viscous friction coefficient. The 
three effects as well as their combined effect is visualized in Figure 2.17.

The Stribeck effect however has a major effect only for small velocities and

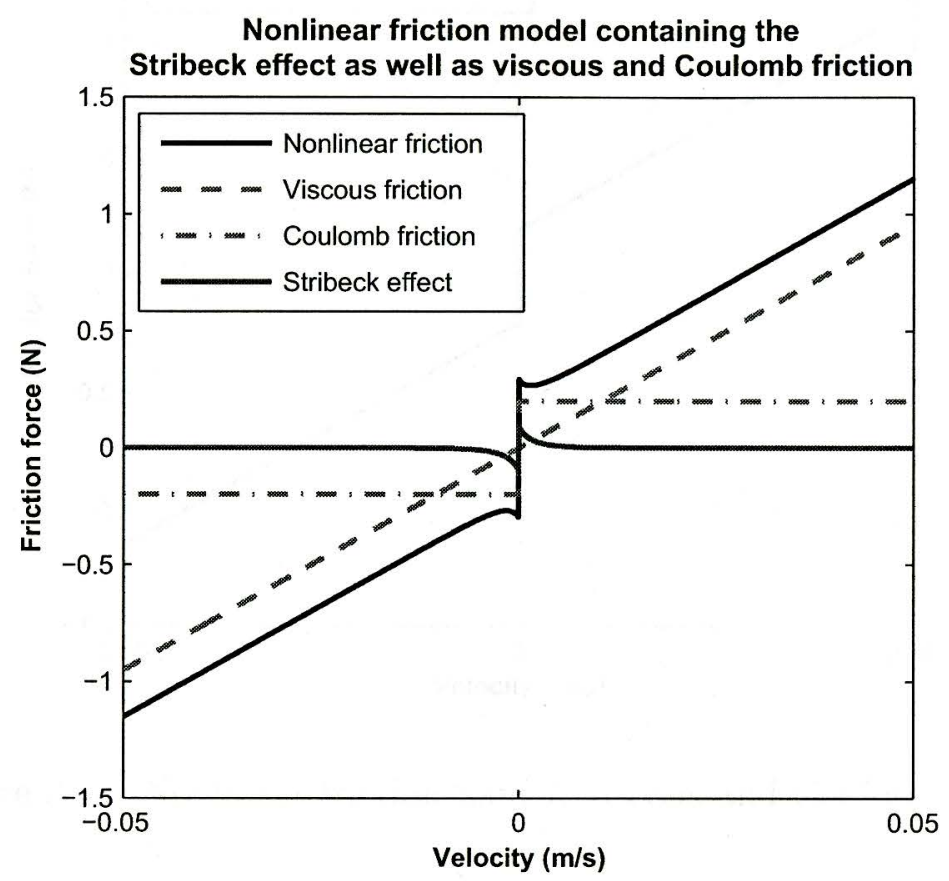

Figure 2.17. Nonlinear friction, Stribeck effect, viscous and Coulomb friction

therefore can be neglected in this belt drive case. This changes the nonlinear friction model from equation 2.15 to equation 2.16 , leaving only the viscous and the Coulomb friction.

$$
F_{f}=b v+\operatorname{sign}(v) F_{c}
$$

Similarly a friction torque can be expressed by changing the forces in the equation to torques, the linear velocity to angular velocity and by adjusting the parameters. Figure 2.18 shows the friction model described in equation 2.16.

In order to model the nonlinear friction and simulate it in Simulink the strictly linear State Space block can not be used anymore to represent the now nonlinear plant. To be able to account for the nonlinearity each equation from 


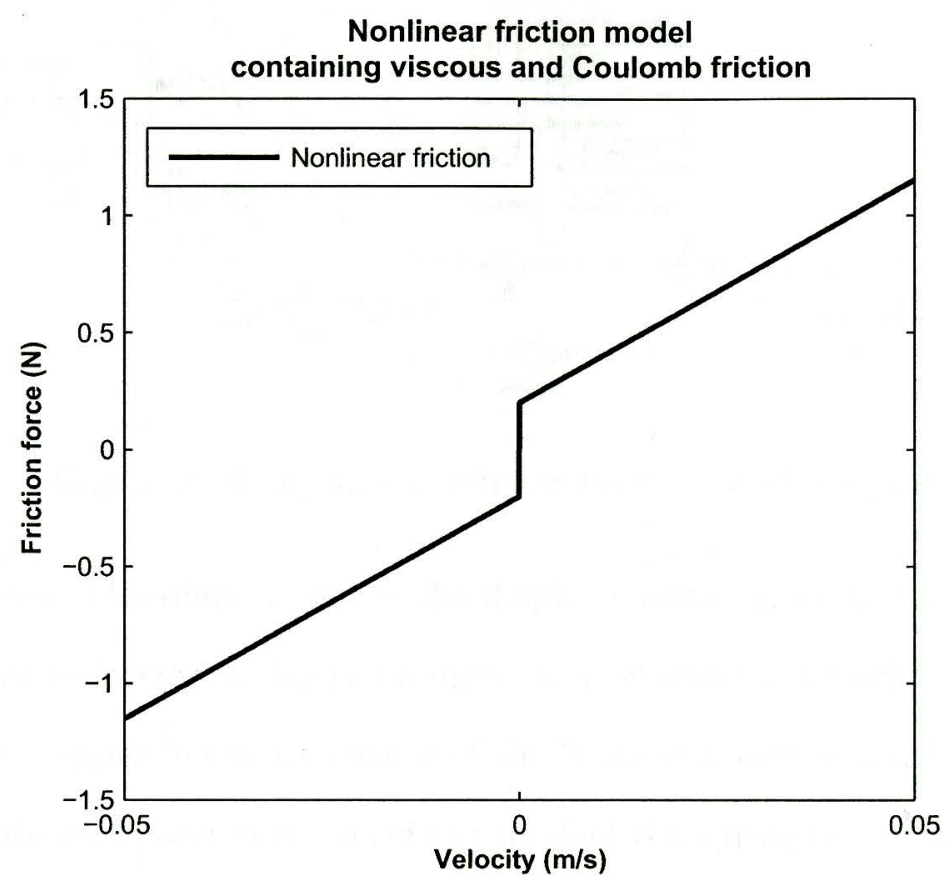

Figure 2.18. Nonlinear friction model, viscous and Coulomb friction

the set of equations of motion has to be represented as a block diagram. Simulink offers all necessary blocks like integrators, gains and summation blocks to be able to model differential equations. To be able to do so the equations of motions are brought into a form which is easy to represent in Simulink block diagrams. Equations 2.17 through 2.19 show the set of equations of motion in this form utilizing the elements of the state space vector to represent the displacement, velocity and acceleration of the original coordinates shown in equation 2.6.

$$
\begin{aligned}
& \dot{x_{2}}=\frac{1}{J_{1}}\left(-\left(x_{1} r-x_{3}\right) r k_{1}+\left(x_{5}-x_{1}\right) r^{2} k_{3}-\tau_{f_{1}}+\tau_{M}\right) \\
& \dot{x_{4}}=\frac{1}{M}\left(\left(x_{1} r-x_{3}\right) k_{1}-\left(x_{3}-x_{5} r\right) k_{2}-F_{f}\right) \\
& \dot{x_{6}}=\frac{1}{J_{2}}\left(\left(x_{3}-x_{5} r\right) r k_{2}-\left(x_{5}-x_{1}\right) r^{2} k_{3}-\tau_{f_{2}}\right)
\end{aligned}
$$

Figure 2.19, shows as an example, how equation 2.17 is transferred into a Simulink 


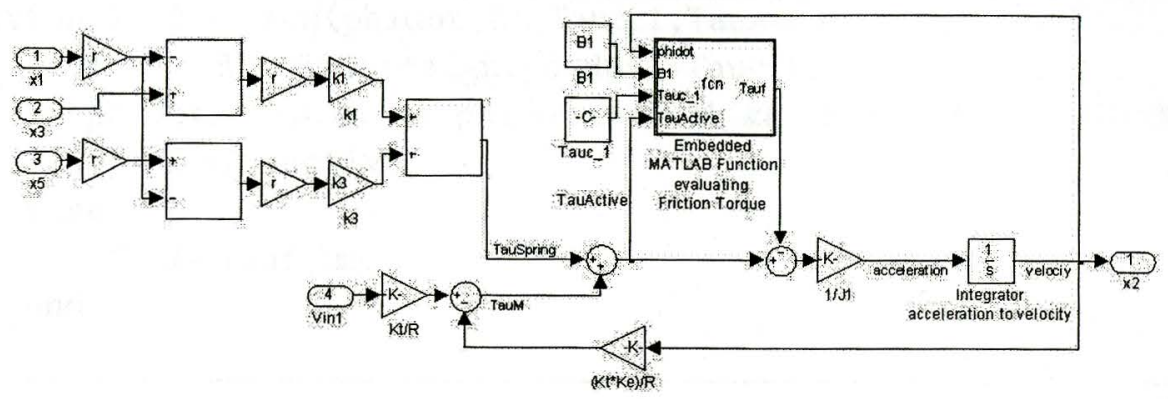

Figure 2.19. $\varphi_{1}$ axis nonlinear friction block diagram

block diagram. The diagram takes the displacements $x_{1}, x_{3}$ and $x_{5}$ as well as the Voltage input to the motor $V_{i n}$ as an input and returns the velocity $x_{2}$ of the motor pulley. In the upper left hand corner of the block diagram it can be seen how the displacements $x_{1}, x_{3}$ and $x_{5}$ are used to calculate the spring torque which is applied to the motor pulley through the belt. This part of the block diagram represents the first two terms in the bracket of equation 2.17. In the lower part of the diagram is shown how the input Voltage $V_{\text {in }}$ as well as the fed back velocity $x_{2}$ are used to calculate the motor torque $\tau_{M}$ according to equation 2.4. Finally the Embedded Matlab function in the upper half of the block diagram is computing the nonlinear friction torque $\tau_{f_{1}}$. This function is taking the motor pulley velocity $x_{2}$, the two constants $B_{1}$ and $\tau_{c}$, which are the viscous friction coefficient and the Coulomb torque respectively, as well as the sum of the spring and the motor torque (the active torque acting on the pulley) as inputs and returns the friction torque $\tau_{f_{1}}$. Table 2.2 shows the code that is being computed while executing that function.

The function is first calculating a temporary friction torque $\tau_{f_{1_{\text {temp }}}}$. Friction torques and friction forces have the characteristic to only oppose a motion, however they would not actually change the direction of the motion, since it is a passive force/torque. This is where the coulomb friction model creates a problem if not implemented properly. The offset values of friction force/torque close to zero may 


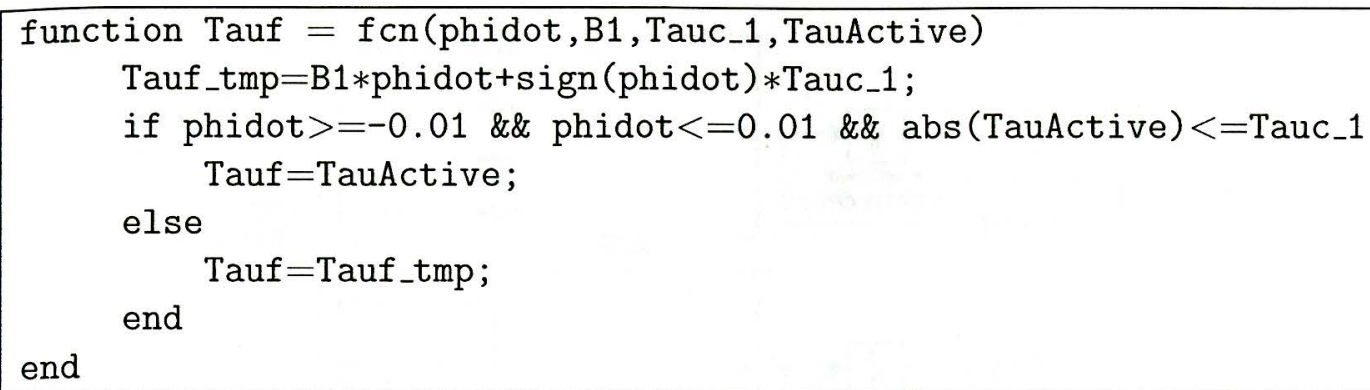

Table 2.2. Code to calculate nonlinear friction

actually change the direction of motion. This is what the if-loop in the function is preventing, it sets the actual friction torque if greater then the active torque (in this case the sum out of motor torque and spring torque) in a velocity band close to zero equal to the active torque in order to cancel out the acting torques and to prevent a change in direction of motion. This friction torque is then being subtracted from the acting torques, which completes the bracket expression. The result of that substraction is then divided by $J_{1}$, which according to equation 2.17 then gives the acceleration of the motor pulley. The acceleration is then integrated to obtain the velocity.

A similar block diagram is generated for each one of the equations of motion, with each having a function to account for the nonlinear friction occurring in that axis. Those 3 block diagrams are then assembled together as subsystems as shown in Figure 2.20.

In this setup the output velocities from each axis block diagram are integrated to obtain the position, which completes together with the velocities the whole state vector. The position is also fed back as the input to the axis block diagrams. The block diagram in Figure 2.20 is again stored in a sub system to get a similar open loop simulation setup (Figure 2.21) as in the linear case. The motor gain seen in this block diagram is the amplifier gain, which is also present in the real system, which in the linear case is included in the input vector $\mathbf{b}$. 


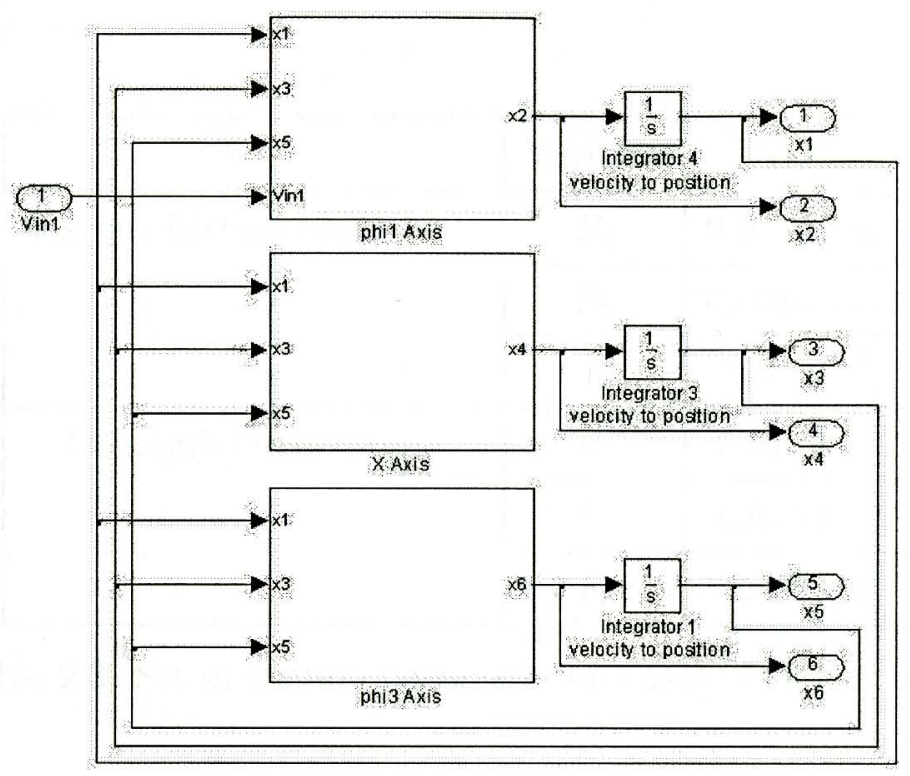

Figure 2.20. nonlinear axis assembled to form the nonlinear plant model

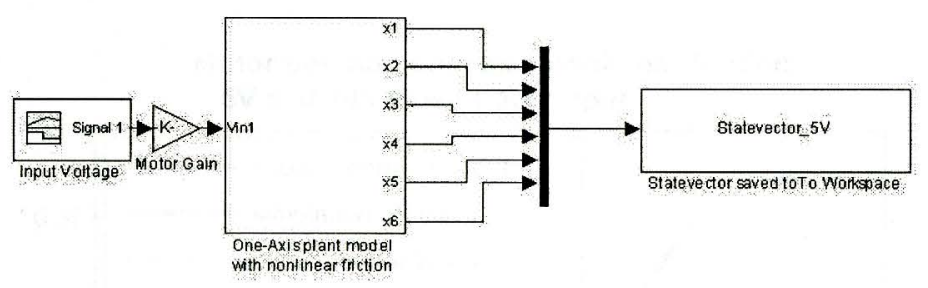

Figure 2.21. nonlinear one-axis open loop simulation setup

With this nonlinear model of the plant it should now be possible to find one set of parameters including the Coulomb friction force/torques that makes it possible to match the $5 \mathrm{~V}$ as well as the $10 \mathrm{~V}$ experimental data. The set of friction parameters that were found, that made this possible are given in Table 2.3.

Figures 2.22 and 2.23 show the results of the nonlinear simulation with the above stated friction parameters compared to the experimental data. It can be seen, that now both the $5 \mathrm{~V}$ and the $10 \mathrm{~V}$ input experimental data can be matched well in contrast to what could be experienced with the linear friction simulation (Figures 2.15 and 2.16). 


\begin{tabular}{|c|c|c|}
\hline & Variable & Value \\
\hline Viscous friction coefficients & $B_{1}$ & $0.0016 \mathrm{Nm} s$ \\
\cline { 2 - 3 } & $B_{2}$ & $0.0016 \mathrm{Nm} s$ \\
\cline { 2 - 3 } & $b$ & $19.0424 \frac{\mathrm{Ns}}{\mathrm{m}}$ \\
\hline \multirow{3}{*}{ Coulomb force/torque } & $\tau_{c_{1}}$ & $0.0150 \mathrm{Nm}$ \\
\cline { 2 - 3 } & $\tau_{c_{2}}$ & $0.0150 \mathrm{Nm}$ \\
\cline { 2 - 3 } & $F_{c}$ & $0.2000 \mathrm{~N}$ \\
\hline
\end{tabular}

Table 2.3. Set of friction parameter one-axis nonlinear model

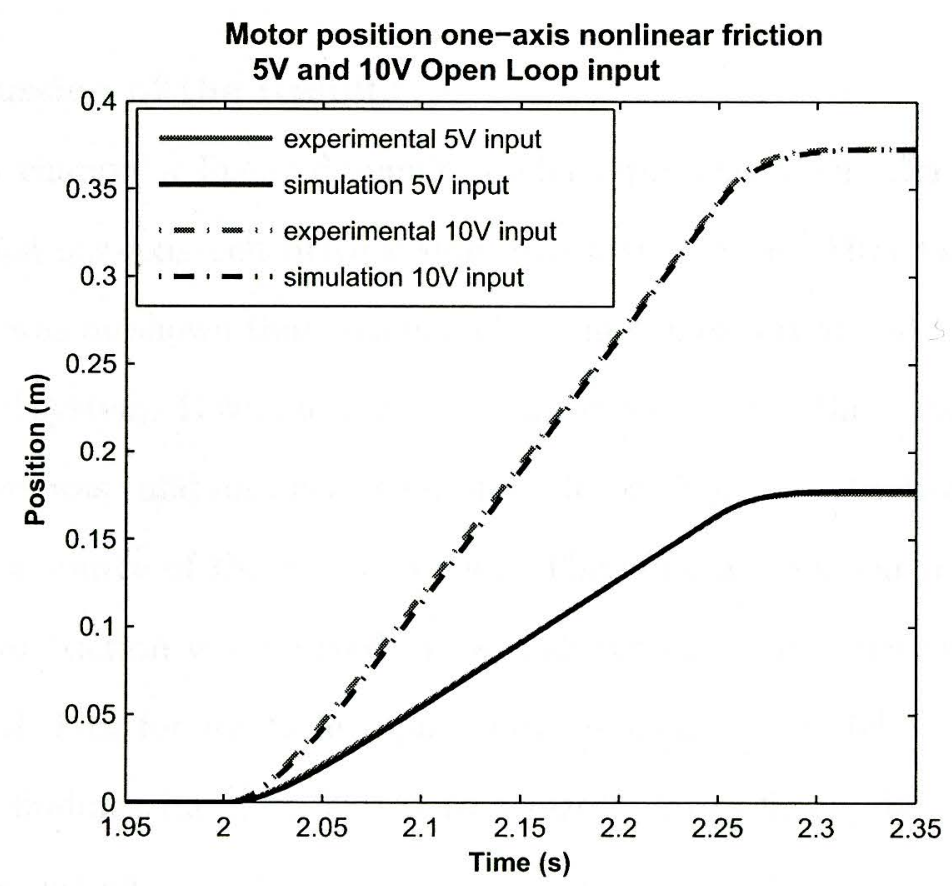

Figure 2.22. 


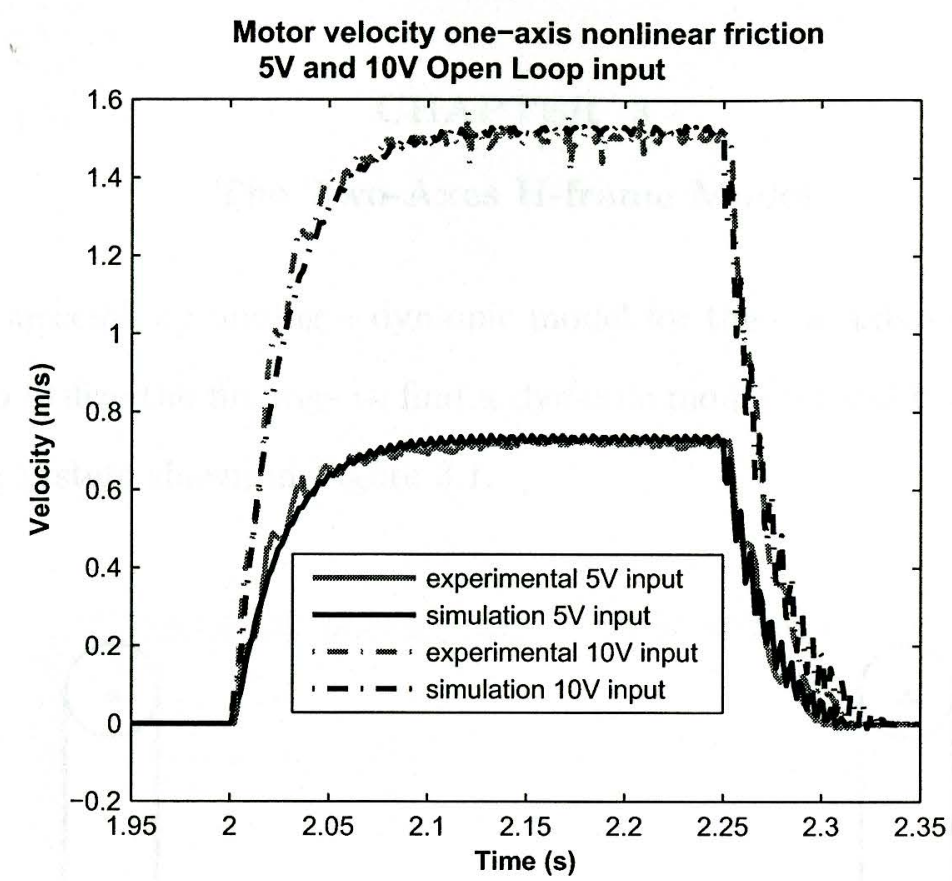

Figure 2.23.

\subsection{Discussion of the Results}

In this chapter a linear dynamic model representing the dynamic behavior of the studied one-axis belt drive system was first derived. After parameters were determined was be shown that this model can match experimental results obtained from the real system. However due to nonlinear behavior of the system this one set of parameters was valid for only one input voltage. Nonlinear friction was found to be the major source of the nonlinearities. Therefore a nonlinear model reflecting the nonlinear friction was derived. It was shown that with this nonlinear model experimental data for multiple input voltages could be matched in simulation. With those findings the next step is to utilize them to find a dynamic model for the H-frame system. 


\section{CHAPTER 3}

\section{The Two-Axes H-frame Model}

After successfully finding a dynamic model for the one-axis system, the next step was to utilize the findings to find a dynamic model for the H-frame two-axes positioning system shown in Figure 3.1.

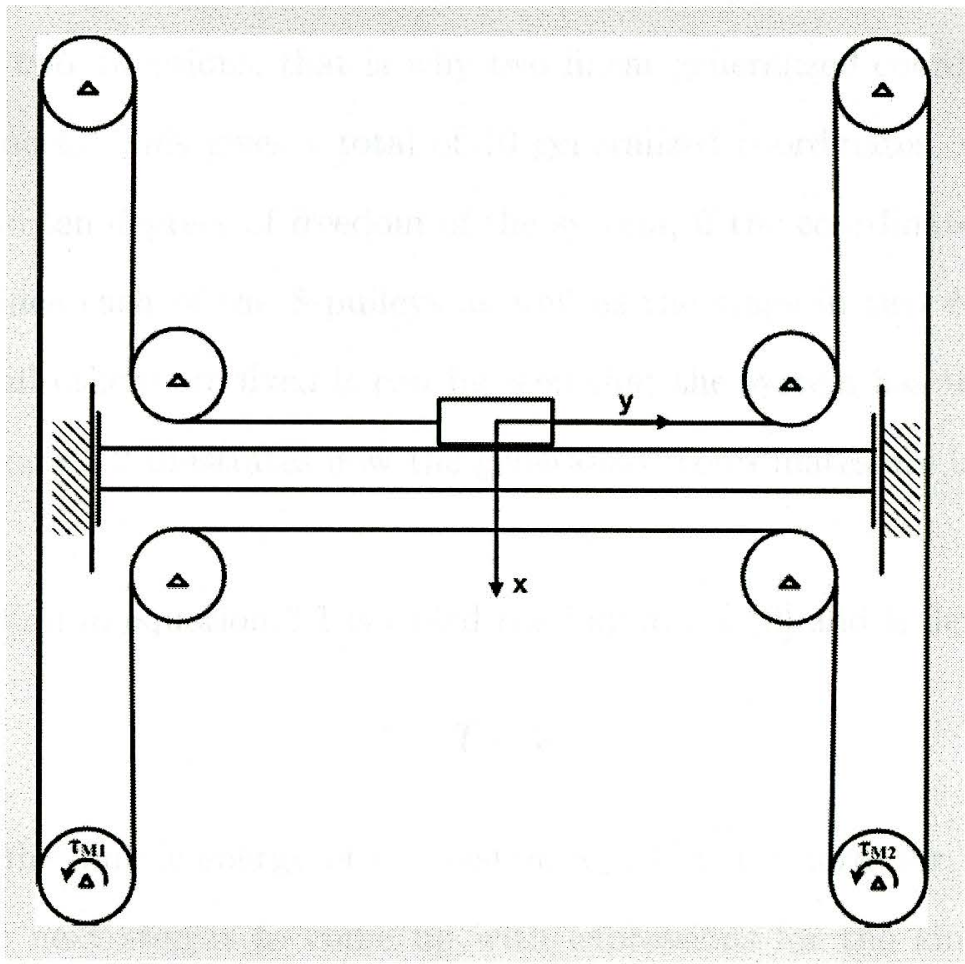

Figure 3.1. H-frame System

\subsection{Equations of Motion for the H-frame System}

For a complex system like the $\mathrm{H}$-frame system the technique of drawing free body diagrams and using d'Alemberts principle is not the best choice, even though theoretically possible using that technique, the derivation will be to complex, which 
can easily lead to errors. The better choice is using Lagrange's Equations (equation 3.1[9]) to get the set of equations of motion.

$$
\frac{d}{d t}\left(\frac{\partial L}{\partial \dot{q}_{j}}\right)-\frac{\partial L}{\partial q_{j}}=Q_{j}^{n c} \quad \text { for } j=1,2, \ldots, n
$$

For this matter in the first step generalized coordinates $q_{j}$ have to be defined. Since the belt sections between the pulleys are again assumed to be springs all the pulleys can to some degree rotate freely from each other. Therefore generalized angular coordinates $\varphi_{1}$ to $\varphi_{8}$ are assigned to the pulleys. Additionally the stage can move in two directions, that is why two linear generalized coordinates $x$ and $y$ are introduced. This gives a total of 10 generalized coordinates, which should correspond to ten degrees of freedom of the system, if the coordinates are chosen correctly. Since each of the 8 pulleys as well as the stage in two directions can move while all others are fixed it can be seen that the system has ten degrees of freedom. Figure 3.2 illustrates how the generalized coordinates are introduced to the system.

The L-function in equation 3.1 is called the Lagrangian[9] and is defined as

$$
L=T-V
$$

Where $T$ is the kinetic energy of the system and $V$ is the term for the potential energy. The next step is to come up with expressions for the kinetic and the potential energy.

The kinetic energy of the whole system can be summed up from the kinetic energy of every element containing inertia, in this case all 8 pulleys as well as the stage, where the stage has kinetic energy in y direction (only the cart) and one in $\mathrm{x}$ direction (the bridge including the cart). Those kinetic energy terms turn out to be: 


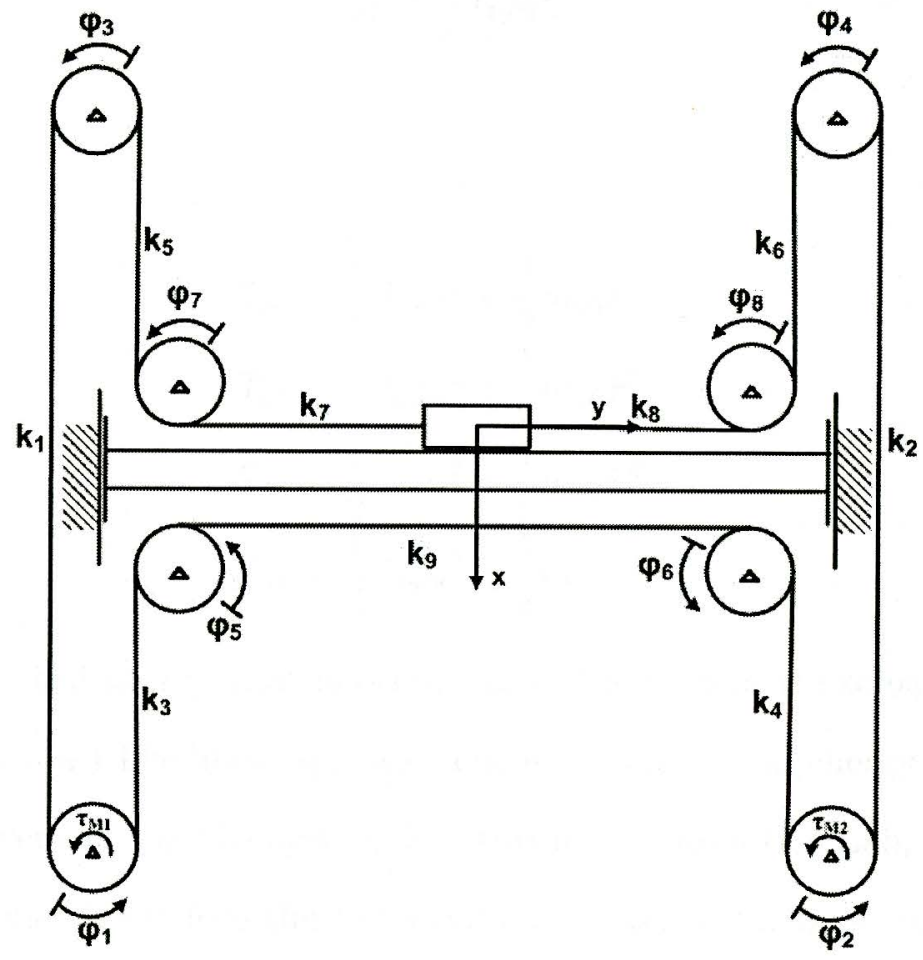

Figure 3.2. Generalized coordinates introduced to the H-frame System

Cart (only in y direction)

$$
T_{\text {cart }}=\frac{1}{2} M_{\text {cart }} \dot{y}^{2}
$$

Bridge (including the Cart)

$$
T_{\text {bridge }}=\frac{1}{2} M_{\text {bridge }} \dot{x}^{2}
$$

Pulleys 1-4

$$
\begin{aligned}
T_{p 1} & =\frac{1}{2} J_{1}{\dot{\varphi_{1}}}^{2} \\
T_{p 2} & =\frac{1}{2}{J_{2}}_{\dot{\varphi}_{2}}{ }^{2} \\
T_{p 3} & =\frac{1}{2} J_{3}{\dot{\varphi_{3}}}^{2}
\end{aligned}
$$




$$
T_{p 4}=\frac{1}{2} J_{4} \dot{\varphi}_{4}^{2}
$$

\section{Pulleys 5-8}

$$
\begin{aligned}
T_{p 5} & =\frac{1}{2} J_{5} \dot{\varphi}_{5}^{2}+\frac{1}{2} m_{p 5} \dot{x}^{2} \\
T_{p 6} & =\frac{1}{2} J_{6} \dot{\varphi}_{6}{ }^{2}+\frac{1}{2} m_{p 6} \dot{x}^{2} \\
T_{p 7} & =\frac{1}{2} J_{7} \dot{\varphi}_{7}{ }^{2}+\frac{1}{2} m_{p 7} \dot{x}^{2} \\
T_{p 8} & =\frac{1}{2} J_{8} \dot{\varphi}_{8}{ }^{2}+\frac{1}{2} m_{p 8} \dot{x}^{2}
\end{aligned}
$$

The potential energy that is occurring in the system is exclusively stored in the belts, which act like linear springs. There is no potential energy due to gravity since the movement, as the system is setup in the Robotics Lab, is only in the horizontal plane. Therefore the potential energy term can be summed up of the potential energy stored in the 9 belt sections. Those belt sections are the left and right long outer sections $\left(k_{1}\right.$ and $\left.k_{2}\right)$, the 4 shorter belt sections between the bridge pulleys and the 4 corner pulleys $\left(k_{3}-k_{6}\right)$, the shorter parallel belt sections in between the bridge pulleys and the cart $\left(k_{7}\right.$ and $\left.k_{8}\right)$ and the long belt section parallel to the bridge $\left(k_{9}\right)$. From now on we will refer to them as belt sections 1 through 9 according to their stiffness $k_{i} i=1,2, \ldots, 9$ as shown in Figure 3.2. The potential energies stored in the belt sections are:

\section{Belt Section 1 and 2}

$$
\begin{aligned}
V_{1} & =\frac{1}{2} k_{1}\left(\varphi_{1} r-\varphi_{3} r\right)^{2} \\
V_{2} & =\frac{1}{2} k_{2}\left(-\varphi_{2} r+\varphi_{4} r\right)^{2}
\end{aligned}
$$

Belt Sections 3-6

$$
V_{3}=\frac{1}{2} k_{3}\left(-\varphi_{1} r-\varphi_{5} r-x\right)^{2}
$$




$$
\begin{aligned}
V_{4} & =\frac{1}{2} k_{4}\left(\varphi_{2} r+\varphi_{6} r-x\right)^{2} \\
V_{5} & =\frac{1}{2} k_{5}\left(\varphi_{3} r+\varphi_{7} r+x\right)^{2} \\
V_{6} & =\frac{1}{2} k_{6}\left(-\varphi_{4} r-\varphi_{8} r+x\right)^{2}
\end{aligned}
$$

Belt Sections 7-8

$$
\begin{aligned}
V_{7} & =\frac{1}{2} k_{7}\left(-\varphi_{7} r+y\right)^{2} \\
V_{8} & =\frac{1}{2} k_{8}\left(\varphi_{8} r-y\right)^{2}
\end{aligned}
$$

\section{Belt Section 9}

$$
V_{9}=\frac{1}{2} k_{9}\left(\varphi_{5} r-\varphi_{6} r\right)^{2}
$$

Using the results from equations 3.3-3.21 the Lagrangian can be written as:

$$
\begin{aligned}
L= & \frac{1}{2} M_{\text {cart }} \dot{y}^{2}+\frac{1}{2} M_{b r i d g e} \dot{x}^{2}+\frac{1}{2} J_{1} \dot{\varphi}_{1}{ }^{2}+\frac{1}{2} J_{2} \dot{\varphi}_{2}^{2}+\frac{1}{2} J_{3} \dot{\varphi}_{3}{ }^{2}+\frac{1}{2} J_{4} \dot{\varphi}_{4}{ }^{2} \\
& +\frac{1}{2} J_{5} \dot{\varphi}_{5}{ }^{2}+\frac{1}{2} m_{p 5} \dot{x}^{2}+\frac{1}{2} J_{6} \dot{\varphi}_{6}{ }^{2}+\frac{1}{2} m_{p 6} \dot{x}^{2} \\
& +\frac{1}{2} J_{7} \dot{\varphi}_{7}{ }^{2}+\frac{1}{2} m_{p 7} \dot{x}^{2}+\frac{1}{2} J_{8} \dot{\varphi}_{8}{ }^{2}+\frac{1}{2} m_{p 8} \dot{x}^{2} \\
& -\frac{1}{2} k_{1}\left(\varphi_{1} r-\varphi_{3} r\right)^{2}-\frac{1}{2} k_{2}\left(-\varphi_{2} r+\varphi_{4} r\right)^{2} \\
& -\frac{1}{2} k_{3}\left(-\varphi_{1} r-\varphi_{5} r-x\right)^{2}-\frac{1}{2} k_{4}\left(\varphi_{2} r+\varphi_{6} r-x\right)^{2} \\
& -\frac{1}{2} k_{5}\left(\varphi_{3} r+\varphi_{7} r+x\right)^{2}-\frac{1}{2} k_{6}\left(-\varphi_{4} r-\varphi_{8} r+x\right)^{2} \\
& -\frac{1}{2} k_{7}\left(-\varphi_{7} r+y\right)^{2}-\frac{1}{2} k_{8}\left(\varphi_{8} r-y\right)^{2}-\frac{1}{2} k_{9}\left(\varphi_{5} r-\varphi_{6} r\right)^{2}
\end{aligned}
$$

After deriving the Lagrangian, an expression for the non-conservative generalized forces $Q_{j}^{n c}$ has to be found. The non-conservative forces are defined by the equation $3.23[9]$

$$
Q_{j}^{n c}=\sum_{k=1}^{n} \vec{F}_{k}^{n c} \frac{\partial \vec{r}_{k}}{\partial q_{j}}
$$


and can be found by looking at the virtual work done by those non-conservative forces through

$$
Q_{j}^{n c}=\frac{\delta W^{n c}}{\delta q_{j}} \quad \text { for } \quad j=1,2, \ldots, 10 . \quad[9]
$$

Where $\delta W^{n c}$ is the non-conservative virtual work and $\delta q_{j} \quad($ for $\quad j=1,2, \ldots, 10)$ are the possible virtual displacements along the generalized coordinates. The nonconservative forces acting on the $\mathrm{H}$-frame system are the Motor torques $\tau_{M_{1}}$ and $\tau_{M_{2}}$ as well as the friction forces and torques occurring in the sliders and the pulley bearings. Therefore the non-conservative virtual work acting on the system is

$$
\begin{aligned}
\delta W^{n c}= & -\dot{y} b_{c a r t} \delta y-\dot{x} b_{b r i d g e} \delta x+\left(\tau_{M_{1}}-B_{1} \dot{\varphi}_{1}\right) \delta \varphi_{1}+\left(\tau_{M_{2}}-B_{2} \dot{\varphi}_{2}\right) \delta \varphi_{2} \\
& -B_{3} \dot{\varphi}_{3} \delta \varphi_{3}-B_{4} \dot{\varphi}_{4} \delta \varphi_{4}-B_{5} \dot{\varphi}_{5} \delta \varphi_{5}-B_{6} \dot{\varphi}_{6} \delta \varphi_{6} \\
& -B_{7} \dot{\varphi}_{7} \delta \varphi_{7}-B_{8} \dot{\varphi}_{8} \delta \varphi_{8}
\end{aligned}
$$

Where $\tau_{M_{i}}$ is described similar to the one axis model and is given by equation 3.26 .

$$
\tau_{M_{i}}=\frac{K_{t}}{R} V_{i_{i n}}-\frac{K_{t} K_{e}}{R} \dot{\varphi}_{i} \quad i=1,2
$$

Now by calculating the derivatives in equation 3.1 and 3.24 , where $q_{1}$ to $q_{10}$ are $x, y$ and $\varphi_{1}$ to $\varphi_{8}$ respectively, and plugging them into equation 3.1 the set of equation of motions describing the dynamic behavior of the H-frame system can be evaluated. These equations of motions are:

$$
\begin{aligned}
\ddot{x}= & \frac{1}{M_{\text {bridge }}}\left[-\left(k_{3}+k_{4}+k_{5}+k_{6}\right) x-b_{\text {bridge }} \dot{x}\right. \\
& \left.-k_{3} r \varphi_{1}+k_{4} r \varphi_{2}-k_{5} r \varphi_{3}+k_{6} r \varphi_{4}-k_{3} r \varphi_{6}-k_{5} r \varphi_{7}+k_{6} r \varphi_{8}\right] \\
\ddot{y}= & \frac{1}{M_{\text {cart }}}\left[-\left(k_{7}+k_{8}\right) y-b_{\text {cart }} \dot{y}+k_{7} r \varphi_{7}+k_{8} r \varphi_{8}\right]
\end{aligned}
$$




$$
\begin{aligned}
\ddot{\varphi}_{1}= & \frac{1}{J_{1}}\left[-k_{3} r x-\left(k_{1}+k_{3}\right) r^{2} \varphi_{1}-\left(B_{1}+\frac{K_{t} K_{e}}{R}\right) \dot{\varphi}_{1}\right. \\
& \left.+k_{1} r^{2} \varphi_{3}-k_{3} r^{2} \varphi_{5}+\frac{K_{t}}{R} V_{1_{i n}}\right] \\
\ddot{\varphi}_{2}= & \frac{1}{J_{2}}\left[-k_{4} r x-\left(k_{2}+k_{4}\right) r^{2} \varphi_{2}-\left(B_{2}+\frac{K_{t} K_{e}}{R}\right) \dot{\varphi}_{2}\right. \\
& \left.+k_{2} r^{2} \varphi_{4}-k_{4} r^{2} \varphi_{6}+\frac{K_{t}}{R} V_{2_{i n}}\right] \\
\ddot{\varphi}_{3}= & \frac{1}{J_{3}}\left[-k_{5} r x+k_{1} r^{2} \varphi_{1}-\left(k 1+k_{5}\right) r^{2} \varphi_{3}-B_{3} \dot{\varphi}_{3}-k_{5} r^{2} \varphi_{7}\right] \\
\ddot{\varphi}_{4}= & \frac{1}{J_{4}}\left[k_{6} r x+k_{2} r^{2} \varphi_{2}-\left(k_{2}+k_{6}\right) r^{2} \varphi_{4}-B_{4} \dot{\varphi}_{4}-k_{6} r^{2} \varphi_{8}\right] \\
\ddot{\varphi}_{5}= & \frac{1}{J_{5}}\left[-k_{3} r x-k_{3} r^{2} \varphi_{1}-\left(k_{3}+k_{9}\right) r^{2} \varphi_{5}-B_{5} \dot{\varphi}_{5}+k_{9} r^{2} \varphi_{6}\right] \\
\ddot{\varphi}_{6}= & \frac{1}{J_{6}}\left[k_{4} r x-k_{4} r^{2} \varphi_{2}+k_{9} r^{2} \varphi_{5}-\left(k_{4}+k_{9}\right) r^{2} \varphi_{6}-B_{6} \dot{\varphi}_{6}\right] \\
\ddot{\varphi}_{7}= & \frac{1}{J_{7}}\left[-k_{5} r x+k_{7} r y-k_{5} r^{2} \varphi_{3}-\left(k_{5}+k_{7}\right) r^{2} \varphi_{7}-B_{7} \dot{\varphi}_{7}\right] \\
\ddot{\varphi}_{8}= & \frac{1}{J_{8}}\left[k_{6} r x+k_{8} r y-k_{6} r^{2} \varphi_{4}-\left(k_{6}+k_{8}\right) r^{2} \varphi_{8}-B_{8} \dot{\varphi}_{8}\right]
\end{aligned}
$$

This set of equations of motion is again being transformed into state space form [8], for the purpose of simulation.

$$
\begin{aligned}
& \dot{\mathbf{x}}=\mathrm{Ax}+\mathrm{Bu} \\
& \mathbf{y}=\mathrm{Cx}+\mathrm{Du}
\end{aligned}
$$

Where $\mathbf{A}, \mathbf{B}, \mathbf{C}$ and $\mathbf{D}$ are called the system matrix, input matrix, output matrix and feedthrough matrix respectively. The states are defined as followed:

$$
\begin{array}{lllr}
x_{1}=x & x_{2}=\dot{x} & x_{11}=\varphi_{4} & x_{12}=\dot{\varphi}_{4} \\
x_{3}=y & x_{4}=\dot{y} & x_{13}=\varphi_{5} & x_{14}=\dot{\varphi}_{5} \\
x_{5}=\varphi_{1} & x_{6}=\dot{\varphi}_{1} & x_{15}=\varphi_{6} & x_{16}=\dot{\varphi}_{6} \\
x_{7}=\varphi_{2} & x_{8}=\dot{\varphi_{2}} & x_{17}=\varphi_{7} & x_{18}=\dot{\varphi} 7 \\
x_{9}=\varphi_{3} & x_{10}=\dot{\varphi_{3}} & x_{19}=\varphi_{8} & x_{20}=\dot{\varphi_{8}}
\end{array}
$$




\subsection{Determining the Parameters}

Now after a mathematical description of the dynamics of the system is found the next task is to define the parameters in order to be able to simulate the system. For the H-frame the same assumptions are made regarding stiffness and friction, as for the one-axis system. This means that for the stiffness, only the length of the belt sections have to be measured, for the inertia the masses of the cart as well as for the bridge have to be scaled and for the friction the same parameters are used as for the one-axis model. Additionally the correction factors for the good match for $5 \mathrm{~V}$ input, as gained from the testing of the one-axis model are utilized.

\subsection{Verifying the Model by Open Loop Simulation}

After obtaining the dynamic model, simulations have to show if the mathematical description of the system matches the behavior observed in the real system. For this matter open loop simulations are carried out and the resultant data is compared with the data obtained from test runs on the real $\mathrm{H}$-frame system. The open loop simulation is again being done in Simulink, with a very similar setup (see Figure 3.3) as for the one-axis system. The Model is again represented by a state space block in which the A- and b-Matrix of the model are utilized, the c-Matrix in this setup is a 20 by 20 identity matrix, in order to be able to store the whole state vector. Furthermore is unlike in the one-axis case the input to the plant is a 2 by 1 vector.

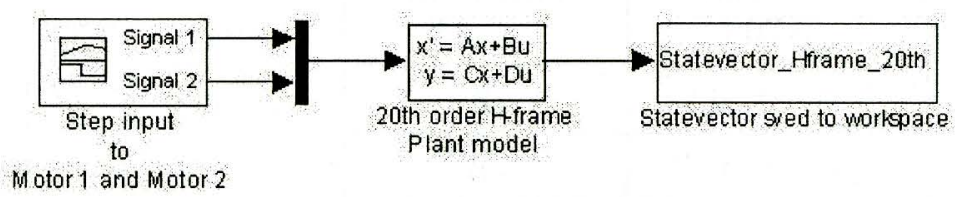

Figure 3.3. H-frame $20^{\text {th }}$-order model open loop simulation setup

For the open loop test a motion in $x$-direction as well as one in $y$-direction is 
being studied. Therefore the system is being applied with $-4 \mathrm{~V}$ to both motors for the $y$-motion and $-4 \mathrm{~V}$ to motor 1 and $4 \mathrm{~V}$ to motor 2 for the $x$-motion. With some further corrections of the parameters based on the experiences from the one-axis tests, a set of parameters could be found to match the dynamic response of the $\mathrm{H}$-frame system well, for the combination of $4 \mathrm{~V}$ and $-4 \mathrm{~V}$ inputs. These parameters are listed in Table 3.1.

The results of the simulation are shown in Figure 3.4-3.7.

\begin{tabular}{|c|c|c|}
\hline \multirow{4}{*}{ Radius } & Variable & Value \\
\hline \multirow{7}{*}{ Stiffness } & $r$ & $0.0194 \mathrm{~m}$ \\
\cline { 2 - 3 } & $k_{1}$ & $1.781 \times 10^{4} \frac{\mathrm{N}}{\mathrm{m}}$ \\
\cline { 2 - 3 } & $k_{2}$ & $1.781 \times 10^{4} \frac{\mathrm{N}}{\mathrm{m}}$ \\
\cline { 2 - 3 } & $k_{3}$ & $2.5013 \times 10^{4} \frac{\mathrm{N}}{\mathrm{m}}$ \\
\cline { 2 - 3 } & $k_{4}$ & $2.5013 \times 10^{4} \frac{\mathrm{N}}{\mathrm{m}}$ \\
\cline { 2 - 3 } & $k_{5}$ & $1.2565 \times 10^{5} \frac{\mathrm{N}}{\mathrm{m}}$ \\
\cline { 2 - 3 } & $k_{6}$ & $1.2565 \times 10^{5} \frac{\mathrm{N}}{\mathrm{m}}$ \\
\cline { 2 - 3 } & $k_{7}$ & $8.4456 \times 10^{4} \frac{\mathrm{N}}{\mathrm{m}}$ \\
\cline { 2 - 3 } & $k_{8}$ & $3.5011 \times 10^{4} \frac{\mathrm{N}}{\mathrm{m}}$ \\
\hline \multirow{7}{*}{ Friction } & $k_{9}$ & $2.2585 \times 10^{4} \frac{\mathrm{N}}{\mathrm{m}}$ \\
\cline { 2 - 3 } & $B_{1}, B_{2}, \ldots, B_{8}$ & $0.0021 \mathrm{Nms}$ \\
\cline { 2 - 3 } & $b_{\text {cart }}$ & $23.2224 \frac{\mathrm{Ns}}{\mathrm{m}}$ \\
\cline { 2 - 3 } & $b_{\text {bridge }}$ & $46.4449 \frac{\mathrm{Ns}}{\mathrm{m}}$ \\
\hline Inertia \& Mass & $J_{1}, J_{2}, \ldots, J_{8}$ & $5.6 \times 10^{-5} \mathrm{Kg} \mathrm{m} \mathrm{m}^{2}$ \\
\cline { 2 - 3 } & $M_{\text {cart }}$ & $0.446 \mathrm{Kg}$ \\
\cline { 2 - 3 } & $M_{\text {bridge }}$ & $4.07 \mathrm{Kg}$ \\
\hline Motor constants & $K_{t}$ & $23 \times 10^{-3} \frac{\mathrm{Nm}}{\mathrm{A}}$ \\
\cline { 2 - 3 } & $K_{e}$ & $23 \times 10^{-3} \frac{\mathrm{V}}{\mathrm{rads}}$ \\
\cline { 2 - 3 } & $R$ & $0.71 \Omega$ \\
\hline
\end{tabular}

Table 3.1. Parameters for the $\mathrm{H}$-frame $20^{\text {th }}$-order model 
Motor angle 1

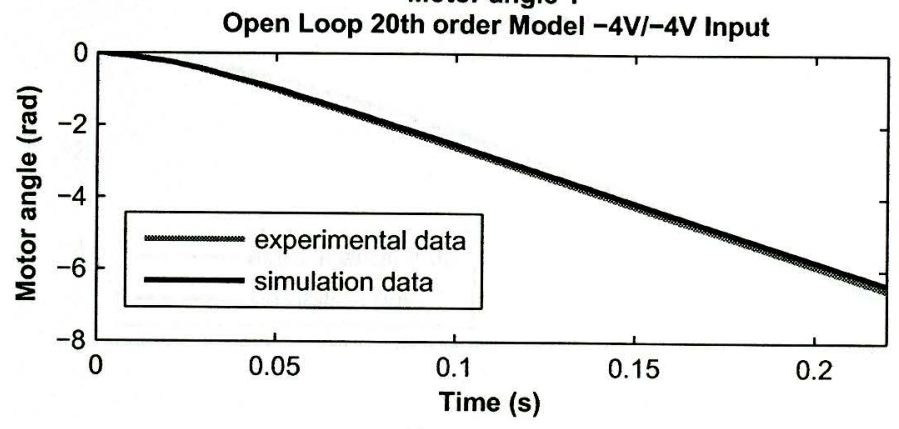

Motor angle 2

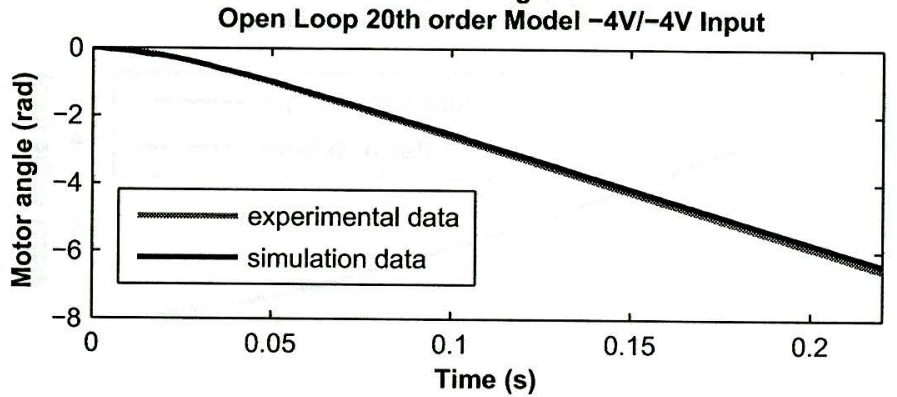

Figure 3.4.

X-position
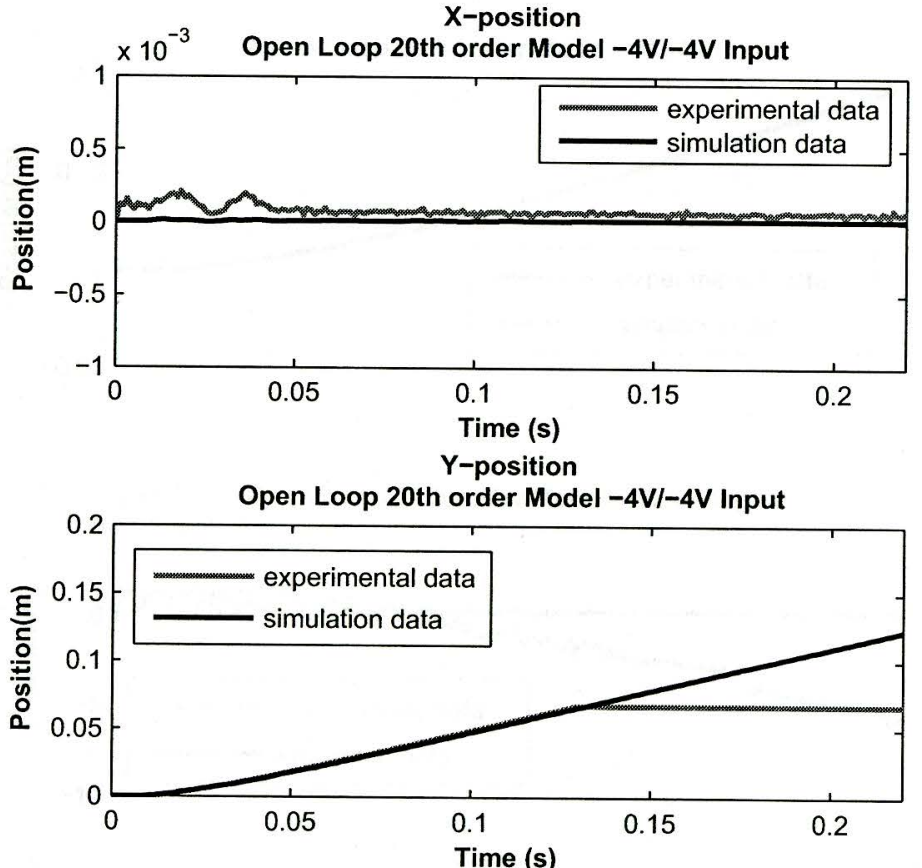

Figure 3.5. 
Motor angle 1

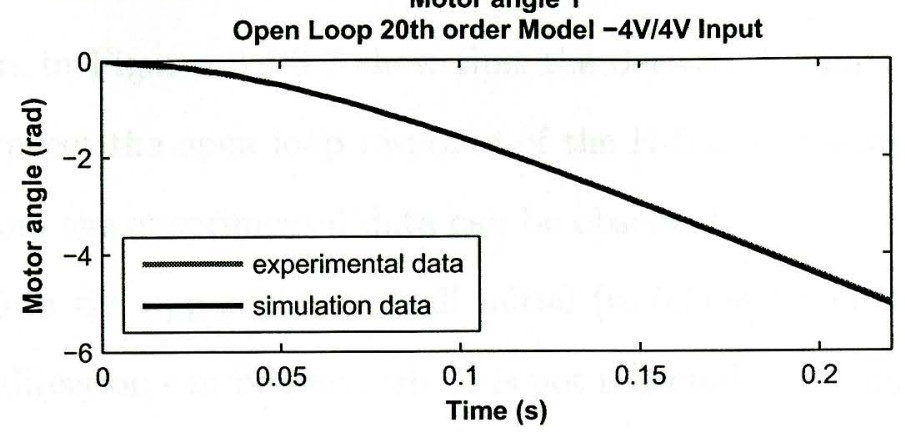

Motor angle 2

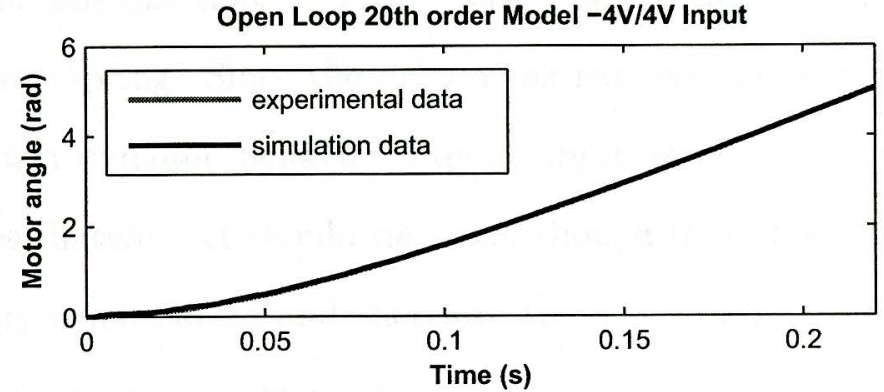

Figure 3.6.
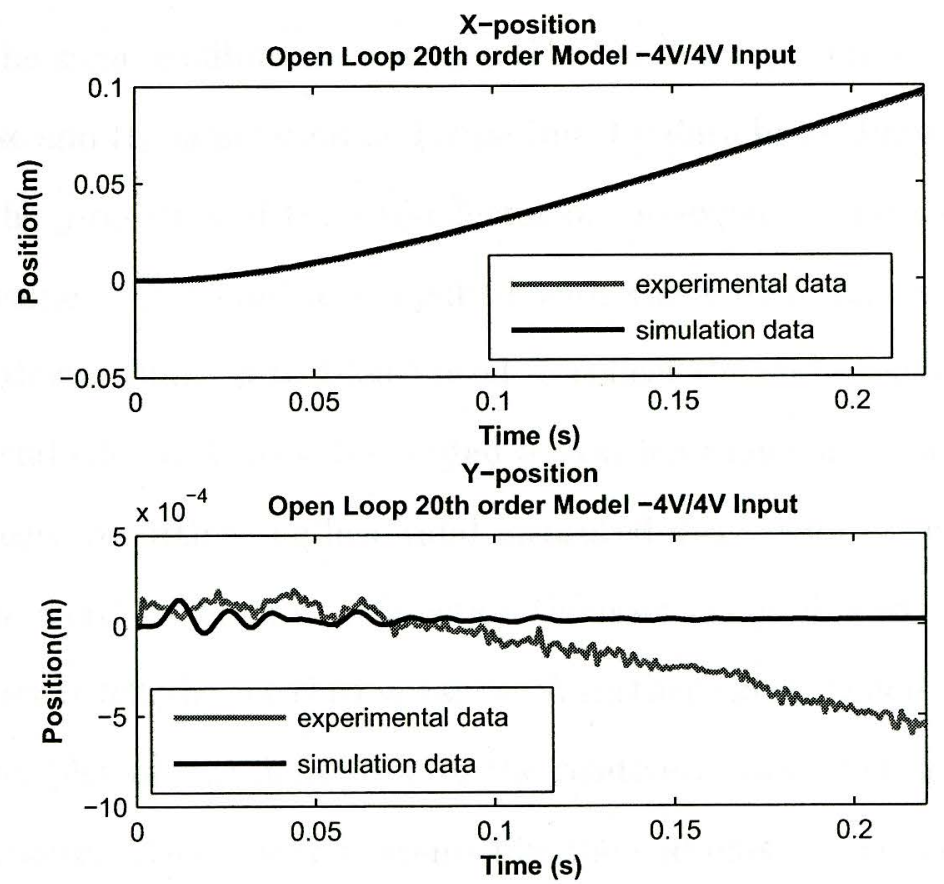

Figure 3.7. 


\subsection{Discussion of the Results}

The plots in Figures 3.4-3.7 show that the derived dynamic model is generally able to reflect the open loop response of the $\mathrm{H}$-frame system. However some deviations from the experimental data can be observed.

In Figure 3.5 in the upper plot a small initial $(\approx 0.2 \mathrm{~mm})$ oscillation of the endeffector in $x$-direction can be seen, which is not reflected in the model in the same amount. Deviations like that could be caused by the assumption that the belt is a massless linear spring. Since the belt in the real system does have a mass and therefore its own dynamic behavior lateral vibration could occur, which lead to those initial oscillations. It should be noted though that those initial oscillations are comparably small effects and therefore the assumption to model the belt as massless linear springs remains valid.

Also the end-effector does not return to a perfect $x=0$ position after that oscillation. This is probably an effect of nonlinear friction, which lets the end-effector not return to the same equilibrium position. For the $y$-axis, there is no oscillation in the response and the simulation and experimental data have almost perfect match. However, the $y$-position of the end-effector of the experimental data saturates at a certain value. This can be explained with the measuring range of the laser measuring device (limited to $10 \mathrm{~cm}$ ) used to collect the end-effector position data. When the end-effector leaves the limited measuring range of the measuring device the laser keeps reporting the last valid measured displacement. Even though the end-effector keeps traveling in $y$-direction the laser is not able to report the actual position after it left the measuring range. A further effect, which can be observed in the upper plot of this figure due to the relatively small $x$-scale, is the random measuring noise. This is why it seems like the end-effector oscillates with a very small amplitude around its steady state value, however since the measurement sig- 
nal gets perturbed by noise a statement to wether there is an oscillation with such a small amplitude or not can not be made with certainty.

Another deviation of simulated and experimental response, which is to be pointed out here, is in the bottom plot of Figure 3.7. In this plot the amplitude of the beginning oscillation is reflected through the simulation, but the shape looks different. Furthermore the experimental data shows a motion of the end-effector in $y$-direction $(0.5 \mathrm{~mm}$ at $0.2 \mathrm{sec})$ while the simulated $y$-position settles at zero. This is most likely cause by a slight difference of the two used motors in the real system, since two motors are never exactly alike. Again the difference in the $x$-scale for both of the plots in this figure has to be considered. The amount of motion in $y$-direction (sub $\mathrm{mm}$ ) is comparably small to the motion in $x$-direction that can be experienced at the same time.

It should also be noted that even though not verified at this point, it is expected that those good results again are only valid for one set of input voltages as it was experienced for the one-axis case. So that for good results for multiple sets of input voltages nonlinear friction has to be considered also for the H-frame dynamic model. 


\section{CHAPTER 4}

\section{The simplified 8th order Model}

Even though the dynamic model with a 20x20 sate space A-Matrix as shown in the last chapter does reflect the dynamic behavior of the H-frame system well, the size of the model could cause problems. Although Simulink does not seem to have a problem simulating a system of that size, for controlling purposes it could be a problem. For example if a state feedback control strategy was chosen to be implemented, an observer would have to run on the controlling personal computer which estimates the 18 not measured states. This is a big computational effort, which could turn out to be difficult to do in real time. It would also generate too much computational effort if this 20th order model was transferred into a Simulink block diagram, in order to simulate the nonlinear friction, similarly as it was done for the one-axis system. Since in that case 10 nonlinear axis have to be modeled and evaluated. For those reasons, in this chapter approximations will be made to reduce the order of the system in order to reduce later computational effort.

\subsection{Assumptions to decrease the Order of the Model}

In order to reduce the order of the system we could assume that the inertia of all the pulleys not driven by a motor is lumped into the two motor pulleys. So that

$$
J_{M_{i}}=4 J_{p} \quad \text { for } \quad i=1,2
$$

where $J_{M_{i}}$ are the new inertias of the motor pulleys and $J_{p}$ are the inertias of the single pulleys as proposed in the previous chapter. However before doing that, a close look at the actual motion of the $\mathrm{H}$-frame positioning system has to be taken. If the motions in $x$ - and in $y$-direction are compared, this assumption is only valid for motion in $y$-direction. This is because if the system is solely moved 
in the $x$-direction, the cart itself does not move relative to the bridge and therefore the four pulleys attached to the bridge do not rotate either. This in turn means that the rotational inertia of those pulleys, does not effect the overall dynamics of the system for motion in $x$-direction. Hence the assumption made in equation 4.1 would lead to too much rotational inertia for this kind of motion. For motion in the $x$-direction only the inertia of the corner pulleys opposite of the motors, which rotate, have to be lumped into the motor pulleys. So that equation 4.1 changes to

$$
J_{M_{i}}=2 J_{p} \quad \text { for } \quad i=1,2
$$

However for those lumped inertias now only the $x$-direction response would match with the real system response and the y-direction response does not. This is since for motion in $y$-direction the bridge pulleys do turn and therefore the inertias have to be considered. As a solution for this, the missing inertia for the $y$-motion is being lumped into the cart, which moves solely in $y$-direction (Mass of the cart is included in $M_{\text {bridge }}$, however only $M_{\text {cart }}$ will be changed and $M_{\text {bridge }}$ stays unchanged). In order to convert the rotational parameters $J_{p}$ into a translation parameter a conversion factor of $\frac{1}{r^{2}}$ has to be multiplied. So the new mass parameter of the cart is

$$
M_{\text {cart }}=M_{\text {cart }_{20}}+4 J_{p} \frac{1}{r^{2}} .
$$

Where $M_{\text {cart }_{20}}$ is the parameter of the previous $20^{\text {th }}$-order model and $M_{\text {cart }}$ is the parameter of the new simplified $8^{\text {th }}$-order model. In a similar fashion, the friction has to be treated and therefore has to be lumped partially into the motor pulleys and into the cart. Equations 4.4 and 4.5 show the new friction coefficients for the motor pulleys and the cart of the simplified $8^{\text {th }}$-order model.

$$
\begin{aligned}
B_{M_{i}} & =2 B_{p} \quad \text { for } \quad i=1,2 \\
b_{\text {cart }_{8}} & =b_{\text {cart }_{20}}+4 B_{p} \frac{1}{r^{2}}
\end{aligned}
$$


Where again $B_{M_{i}}$ and $b_{\text {cart }_{8}}$ are the viscous friction parameters of the new simplified $8^{\text {th }}$-order model and $B_{p}$ and $b_{\text {cart }_{20}}$ are the viscous friction parameters of the $20^{\text {th }}$ order model as proposed in the previous chapter.

Now since all pulleys except the two motor pulleys are ideal pulleys on which neither inertia forces nor friction or external forces act, they do not have to be considered with generalized coordinates, since they do not effect the dynamic behavior of the system. Therefore only four generalized coordinates have to be introduced to the system, two of which are angular coordinates $\varphi_{1}$ and $\varphi_{2}$ describing the motion of the two motor pulleys. The remaining two are the linear coordinates $x$ and $y$ describing the motion of the end-effector, as shown in Figure 4.1.

Since those 6 pulleys (all except for the motor pulleys) do not have their own degree of freedom anymore, the belt now only forms 3 effective springs. One in between motor pulley 1 and the cart $\left(k_{l}\right)$, another one in between the cart and motor pulley $2\left(k_{r}\right)$ and the third one in between motor pulley 1 and motor pulley $2\left(k_{b}\right)$. Figure 4.1 illustrates this.

The belt stiffness $k_{l}, k_{r}$ and $k_{b}$ can be determined as the effective stiffness of the corresponding serial springs of the prior model. These are given by:

$$
\begin{aligned}
& k_{l}=\frac{k_{1} k_{5} k_{7}}{k_{1} k_{5}+k_{1} k_{7}+k_{7} k_{5}} \\
& k_{r}=\frac{k_{2} k_{6} k_{8}}{k_{2} k_{6}+k_{2} k_{8}+k_{6} k_{8}} \\
& k_{b}=\frac{k_{3} k_{4} k_{9}}{k_{3} k_{9}+k_{3} k_{4}+k_{4} k_{9}}
\end{aligned}
$$

After those preliminary considerations the kinetic and potential energy terms can be derived. 


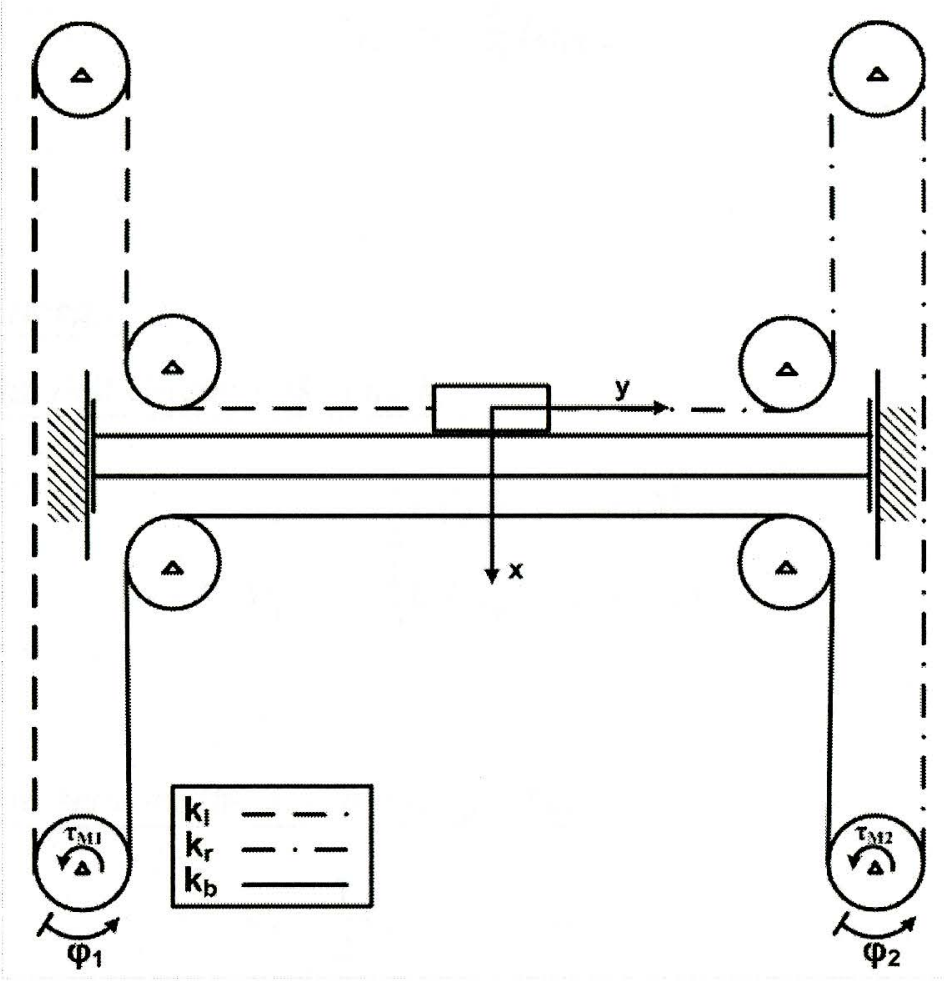

Figure 4.1. H-frame with generalized coordinates for the simplified model

4.2 Equations of motion for the 8th order Model

Kinetic Energy

Cart (only in y-direction)

$$
T_{\text {cart }}=\frac{1}{2} M_{\text {cart }} \dot{y}^{2}
$$

$\underline{\text { Bridge (including cart) }}$

$$
T_{\text {bridge }}=\frac{1}{2} M_{\text {bridge }} \dot{x}^{2}
$$

Motor Pulleys 1 and 2

$$
T_{p_{1}}=\frac{1}{2} J_{M_{1}} \dot{\varphi}_{1}^{2}
$$




$$
T_{p_{2}}=\frac{1}{2} J_{M_{2}} \dot{\varphi}_{2}^{2}
$$

\section{Potential Energy}

Left and Right Belt Sections ( $k_{l}$ and $k_{r}$ )

$$
\begin{aligned}
V_{l} & =\frac{1}{2} k_{l}\left(\varphi_{1} r+x+y\right)^{2} \\
V_{2} & =\frac{1}{2} k_{r}\left(-\varphi_{2} r+x-y\right)^{2}
\end{aligned}
$$

Belt Section in between the Motor pulleys $\left(k_{b}\right)$

$$
V_{b}=\frac{1}{2} k_{b}\left(-\varphi_{1} r+\varphi_{2} r-2 x\right)^{2}
$$

From those terms the Lagrangian $\mathrm{L}$ is assembled

$$
\begin{aligned}
L=T-V= & \frac{1}{2} M_{\text {cart }} \dot{y}^{2}+\frac{1}{2} M_{b r i d g e} \dot{x}^{2}+\frac{1}{2} J_{M_{1}} \dot{\varphi}_{1}{ }^{2}+\frac{1}{2} J_{M_{2}}{\dot{\varphi_{2}}}^{2} \\
& -\frac{1}{2} k_{l}\left(\varphi_{1} r+x+y\right)^{2}-\frac{1}{2} k_{r}\left(-\varphi_{2} r+x-y\right)^{2} \\
& -\frac{1}{2} k_{b}\left(-\varphi_{1} r+\varphi_{2} r-2 x\right)^{2}
\end{aligned}
$$

The next step is to consider the non-conservative forces $Q_{j}^{n c}$. As in the prior model the motor torques $\tau_{M_{i}}$ have to be treated as non-conservative forces and also the friction forces of the cart and the bridge have to be treated the same way as in the prior model. As discussed before, the friction torques in the pulleys only occur in the motor pulleys and the friction coefficients are the lumped frictions coefficients $B_{M_{i}}$ as derived before. Therefore the virtual work acting on this system is given by.

$$
\delta W^{n c}=-\dot{y} b_{c a r t} \delta y-\dot{x} b_{b r i d g e} \delta x+\left(\tau_{M_{1}}-B_{M_{i}} \dot{\varphi}_{1}\right) \delta \varphi_{1}+\left(\tau_{M_{2}}-B_{M_{2}} \dot{\varphi}_{2}\right) \delta \varphi_{2}
$$




$$
T_{p_{2}}=\frac{1}{2} J_{M_{2}} \dot{\varphi}_{2}^{2}
$$

\section{$\underline{\text { Potential Energy }}$}

Left and Right Belt Sections ( $k_{l}$ and $\left.k_{r}\right)$

$$
\begin{aligned}
V_{l} & =\frac{1}{2} k_{l}\left(\varphi_{1} r+x+y\right)^{2} \\
V_{2} & =\frac{1}{2} k_{r}\left(-\varphi_{2} r+x-y\right)^{2}
\end{aligned}
$$

Belt Section in between the Motor pulleys $\left(k_{b}\right)$

$$
V_{b}=\frac{1}{2} k_{b}\left(-\varphi_{1} r+\varphi_{2} r-2 x\right)^{2}
$$

From those terms the Lagrangian $\mathrm{L}$ is assembled

$$
\begin{aligned}
L=T-V= & \frac{1}{2} M_{\text {cart }} \dot{y}^{2}+\frac{1}{2} M_{\text {bridge }} \dot{x}^{2}+\frac{1}{2} J_{M_{1}} \dot{\varphi}_{1}{ }^{2}+\frac{1}{2} J_{M_{2}} \dot{\varphi}_{2}{ }^{2} \\
& -\frac{1}{2} k_{l}\left(\varphi_{1} r+x+y\right)^{2}-\frac{1}{2} k_{r}\left(-\varphi_{2} r+x-y\right)^{2} \\
& -\frac{1}{2} k_{b}\left(-\varphi_{1} r+\varphi_{2} r-2 x\right)^{2}
\end{aligned}
$$

The next step is to consider the non-conservative forces $Q_{j}^{n c}$. As in the prior model the motor torques $\tau_{M_{i}}$ have to be treated as non-conservative forces and also the friction forces of the cart and the bridge have to be treated the same way as in the prior model. As discussed before, the friction torques in the pulleys only occur in the motor pulleys and the friction coefficients are the lumped frictions coefficients $B_{M_{i}}$ as derived before. Therefore the virtual work acting on this system is given by.

$$
\delta W^{n c}=-\dot{y} b_{c a r t} \delta y-\dot{x} b_{b r i d g e} \delta x+\left(\tau_{M_{1}}-B_{M_{i}} \dot{\varphi}_{1}\right) \delta \varphi_{1}+\left(\tau_{M_{2}}-B_{M_{2}} \dot{\varphi}_{2}\right) \delta \varphi_{2}
$$


where the motor torques are described by

$$
\tau_{M_{i}}=\frac{K_{t}}{R} V_{i_{i n}}-\frac{K_{t} K_{e}}{R} \dot{\varphi}_{i} \quad i=1,2
$$

Substituting equations 4.16-4.18 into Lagrange's equation (equation 4.19[9]) where $n=4$ and $q_{1}$ through $q_{4}$ are $x, y, \varphi_{1}$ and $\varphi_{2}$ respectively gives the set of equations of motion (equation 4.20-4.23) that describe the dynamic behavior of this simplified system.

$$
\begin{gathered}
\frac{d}{d t}\left(\frac{\partial L}{\partial \dot{q}_{j}}\right)-\frac{\partial L}{\partial q_{j}}=Q_{j}^{n c} \quad \text { for } j=1,2, \ldots, n \\
\ddot{x}=\frac{1}{M_{b r i d g e}}\left[-\left(k_{r}+k_{l}+4 k_{b}\right) x-b_{b r i d g e} \dot{x}+\left(k_{r}-k_{l}\right) y\right. \\
\ddot{y}=\frac{1}{M_{c a r t}}\left[-\left(-k_{r}+k_{l}\right) x-\left(k_{r}+k_{l}\right) y-b_{c a r t} \dot{y}-k_{l} r \varphi_{1}-k_{r} r \varphi_{2}\right] \\
\ddot{\varphi_{1}=} \frac{1}{J_{M_{1}}}\left[-\left(k_{l}+2 k_{b}\right) r x-k_{l} r y-\left(k_{b}+k_{l}\right) r^{2} \varphi_{1}\right. \\
\left.-\left(B_{M_{1}}+\frac{K_{t} K_{e}}{R}\right) \dot{\varphi}_{1}+k_{b} r^{2} \varphi_{2}+\frac{K_{t}}{R} V_{1_{i n}}\right] \\
\ddot{\varphi_{2}=} \frac{1}{J_{M_{2}}}\left[\left(k_{r}+2 k_{b}\right) r x-k_{r} y+k_{b} r^{2} \varphi_{1}-\left(k_{b}+k_{r}\right) r^{2} \varphi_{2}\right. \\
\left.-\left(B_{M_{2}}+\frac{K_{t} K_{e}}{R}\right) \dot{\varphi}_{2}+\frac{K_{t}}{R} V_{2_{i n}}\right]
\end{gathered}
$$

This set of equations of motion again similar to the 20th order model can be transferred into state space form. However instead of having a 20 by 20 A-Matrix, now the A-Matrix is only of the size 8 by 8 . Hence the state vector is only 8 by 1 with the states being:

$$
x_{1}=x \quad x_{2}=\dot{x}
$$




$$
\begin{array}{ll}
x_{3}=y & x_{4}=\dot{y} \\
x_{5}=\varphi_{1} & x_{6}=\dot{\varphi_{1}} \\
x_{7}=\varphi_{2} & x_{8}=\dot{\varphi_{2}}
\end{array}
$$

The Matrices for this Model are as followed.

\section{$\underline{\text { System Matrix }}$}

$$
\left[\begin{array}{cccccccc}
0 & 1 & 0 & 0 & 0 & 0 & 0 & 0 \\
a_{21} & a_{22} & a_{23} & 0 & a_{25} & 0 & a_{27} & 0 \\
0 & 0 & 0 & 1 & 0 & 0 & 0 & 0 \\
a_{41} & 0 & a_{43} & a_{44} & a_{45} & 0 & a_{47} & 0 \\
0 & 0 & 0 & 0 & 0 & 1 & 0 & 0 \\
a_{61} & 0 & a_{63} & 0 & a_{65} & a_{66} & a_{67} & 0 \\
0 & 0 & 0 & 0 & 0 & 0 & 0 & 1 \\
a_{81} & 0 & a_{83} & 0 & a_{85} & 0 & a_{87} & a_{88}
\end{array}\right]
$$

Where the matrix elements are:

$$
\begin{aligned}
& a_{21}=-\frac{k_{l}+k_{r}+4 k_{b}}{M_{\text {bridge }}} \\
& a_{22}=-\frac{B_{\text {bridge }}}{M_{\text {bridge }}} \\
& a_{23}=-\frac{k_{l}-k_{r}}{M_{\text {bridge }}} \\
& a_{25}=-\frac{k l+2 k b}{M_{\text {bridge }}} r \\
& a_{27}=\frac{k_{r}+2 k_{b}}{M_{\text {bridge }}} r \\
& a_{41}=-\frac{k_{l}-k_{r}}{M_{\text {cart }}} \\
& a_{43}=-\frac{k_{l}+k_{r}}{M_{\text {cart }}} \\
& a_{44}=-\frac{b_{\text {cart }}}{M_{\text {cart }}} \\
& a_{45}=-\frac{b_{\text {cart }}}{M_{\text {cart }}} \\
& a_{47}=-\frac{k_{r}}{M_{\text {cart }} r} \\
& a_{61}=-\frac{k_{l}+2 k_{b}}{J_{M_{1}}} r
\end{aligned}
$$




$$
\begin{aligned}
a_{63} & =-\frac{k_{l}}{J_{M_{1}}} \\
a_{65} & =-\frac{k_{l}+k_{b}}{J_{M_{1}}} r^{2} \\
a_{66} & =-\left(\frac{B_{M_{1}}}{J_{M_{1}}}+\frac{K_{t} K_{e}}{R J_{M_{1}}}\right) \\
a_{67} & =\frac{k_{b}}{J_{M_{1}}} r^{2} \\
a_{81} & =\frac{k_{r}+2 k_{b}}{J_{M_{2}}} r \\
a_{83} & =-\frac{k_{r}}{J_{M_{2}}} \\
a_{85} & =\frac{k_{b}}{J_{M_{2}}} r^{2} \\
a_{87} & =-\frac{k_{r}+k_{b}}{J_{M_{2}}} r^{2} \\
a_{88} & =-\left(\frac{B_{M_{2}}}{J_{M_{2}}}+\frac{K_{t} K_{e}}{R J_{M_{2}}}\right)
\end{aligned}
$$

Input Matrix

Output Matrix

$$
\mathbf{B}=\left[\begin{array}{cc}
0 & 0 \\
0 & 0 \\
0 & 0 \\
0 & 0 \\
0 & 0 \\
\frac{K_{t}}{R J_{M_{1}}} & 0 \\
0 & 0 \\
0 & \frac{K_{t}}{R J_{M_{2}}}
\end{array}\right]
$$

$$
\mathbf{C}=\left[\begin{array}{llllllll}
0 & 0 & 0 & 0 & 1 & 0 & 0 & 0 \\
0 & 0 & 0 & 0 & 0 & 0 & 1 & 0
\end{array}\right]
$$

Feedthrough Matrix

$$
\mathbf{D}=\left[\begin{array}{ll}
0 & 0 \\
0 & 0
\end{array}\right]
$$

\subsection{Verifying the Model by Open Loop Simulation}

The parameters for the this simplified model according to the applied assumptions are displayed in Table 4.1. To verify that the assumptions are right and this simplified model shows the same dynamic behavior as the $20^{\text {th }}$-order model and 


\begin{tabular}{|c|c|c|}
\hline & Variable & Value \\
\hline Radius & $r$ & $0.0194 m$ \\
\hline \multirow[t]{3}{*}{ Stiffness } & $k_{l}$ & $1.3167 \times 10^{4} \frac{\mathrm{N}}{\mathrm{m}}$ \\
\hline & $k_{r}$ & $1.0791 \times 10^{4} \frac{\mathrm{N}}{\mathrm{m}}$ \\
\hline & $k_{b}$ & $8.0492 \times 10^{3} \frac{\mathrm{N}}{\mathrm{m}}$ \\
\hline \multirow[t]{4}{*}{ Friction } & $B_{M 1}$ & $0.0042 \mathrm{Nms}$ \\
\hline & $B_{M 2}$ & $0.0042 \mathrm{Nms}$ \\
\hline & $b_{\text {cart }}$ & $45.5517 \frac{\mathrm{Ns}}{\mathrm{m}}$ \\
\hline & $b_{\text {bridge }}$ & $46.4449 \frac{\mathrm{Ns}}{\mathrm{m}}$ \\
\hline \multirow[t]{4}{*}{ Inertia \& Mass } & $J_{M 1}$ & $1.12 \times 10^{-4} \mathrm{Kg} \mathrm{m}^{2}$ \\
\hline & $J_{M 2}$ & $1.12 \times 10^{-4} \mathrm{Kg} \mathrm{m}^{2}$ \\
\hline & $M_{\text {cart }}$ & $1.0412 \mathrm{Kg}$ \\
\hline & $M_{\text {bridge }}$ & $4.07 \mathrm{Kg}$ \\
\hline \multirow[t]{3}{*}{ Motor constants } & $K_{t}$ & $23 \times 10^{-3} \frac{\mathrm{Nm}}{\mathrm{A}}$ \\
\hline & $K_{e}$ & $23 \times 10^{-3} \frac{V}{r a d s}$ \\
\hline & $R$ & $0.71 \Omega$ \\
\hline
\end{tabular}

Table 4.1. Parameters for the H-frame $8^{\text {th }}$-order model

therefore as the real $\mathrm{H}$-frame system, open loop simulations will be carried out. For those tests the same specifications regarding Voltage input as for the $20^{\text {th }}$-order model are chosen and the resulting data is compared to the experimental as well as the $20^{\text {th }}$-order simulation data. For the Simulink simulation the same block diagram setup (see Figure 3.3 ) as for the $20^{\text {th }}$-order model is used. However now the plant state space matrices in the state space block are those of the $8^{\text {th }}$-order model.

The plots in Figures 4.2 through 4.5 show that the simplified 8th order model has almost the exact same dynamic response as the 20 th order model and therefore also matches the experimental data well. This leads to the conclusion that those 
Motor angle 1 Open Loop 8th order Model vs. 20th order Model -4V/-4V Input

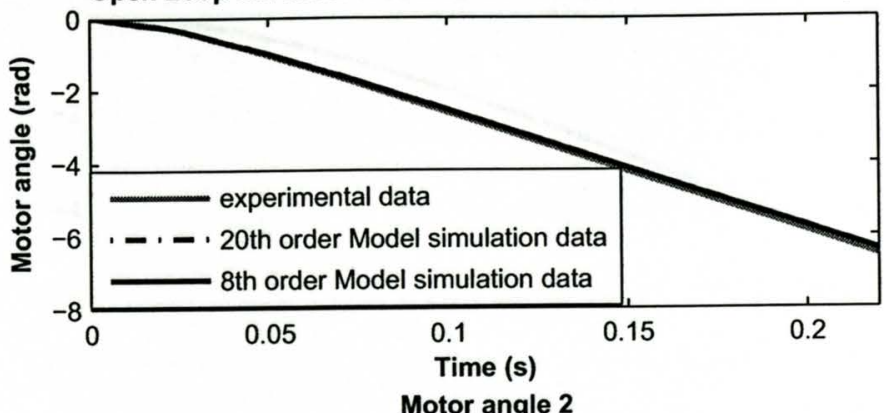

Open Loop 8th order Model vs. 20th order Model $-4 \mathrm{~V} /-4 \mathrm{~V}$ Input

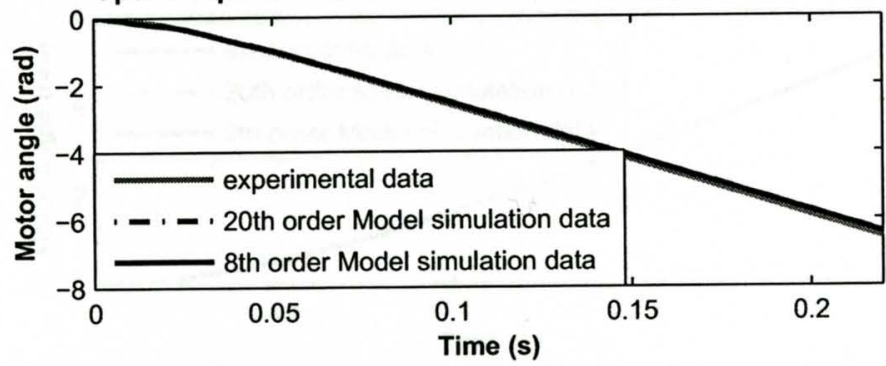

Figure 4.2. Open loop test y-motion motor angles

$$
\text { X-position }
$$
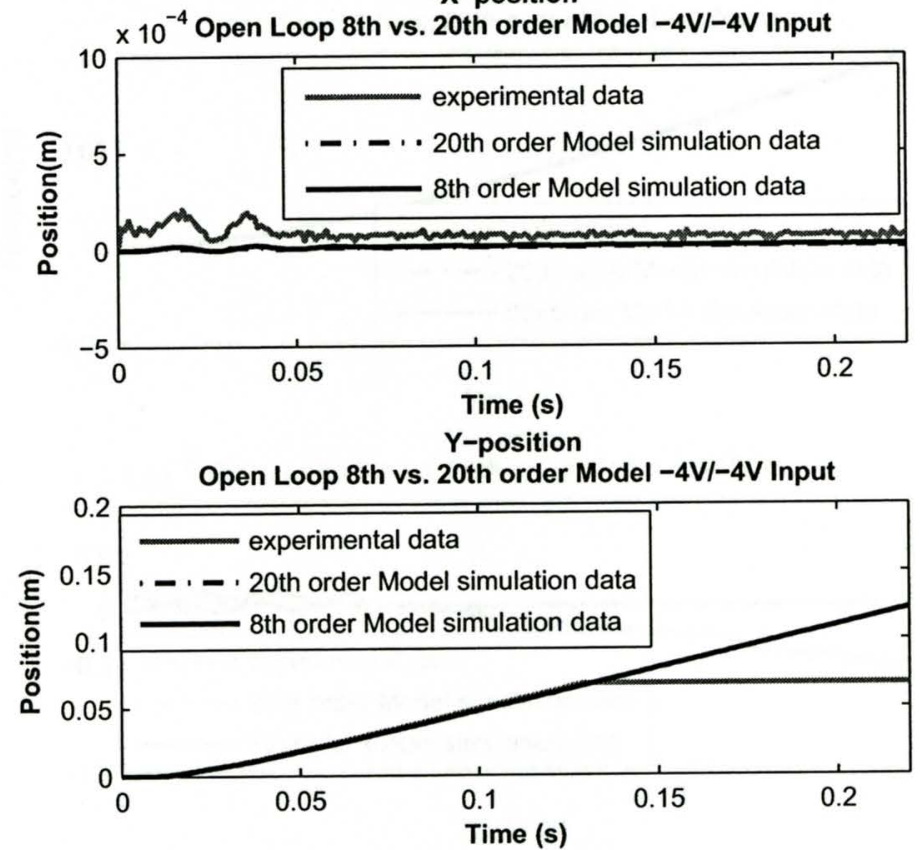

Figure 4.3. Open loop test y-motion end-effector position 


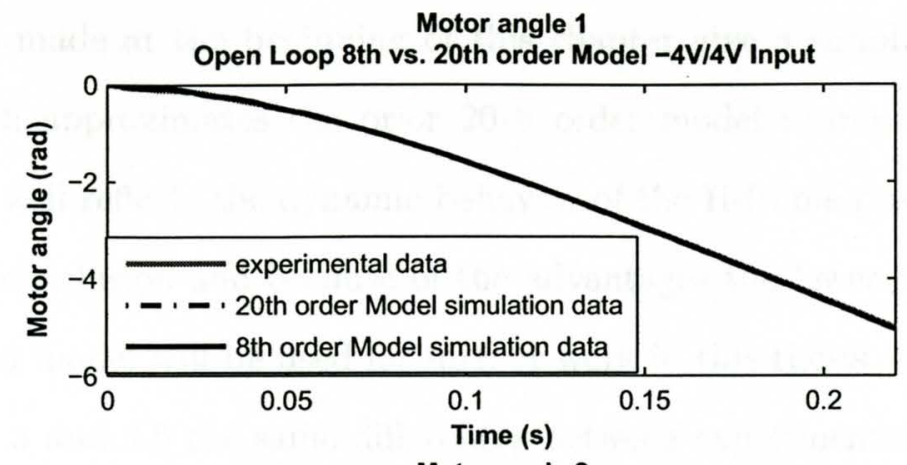

Motor angle 2

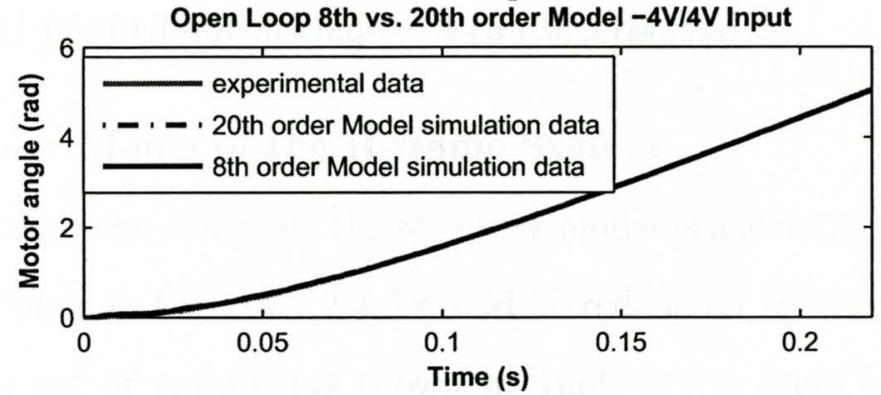

Figure 4.4. Open loop test $\mathrm{x}$-motion motor angles
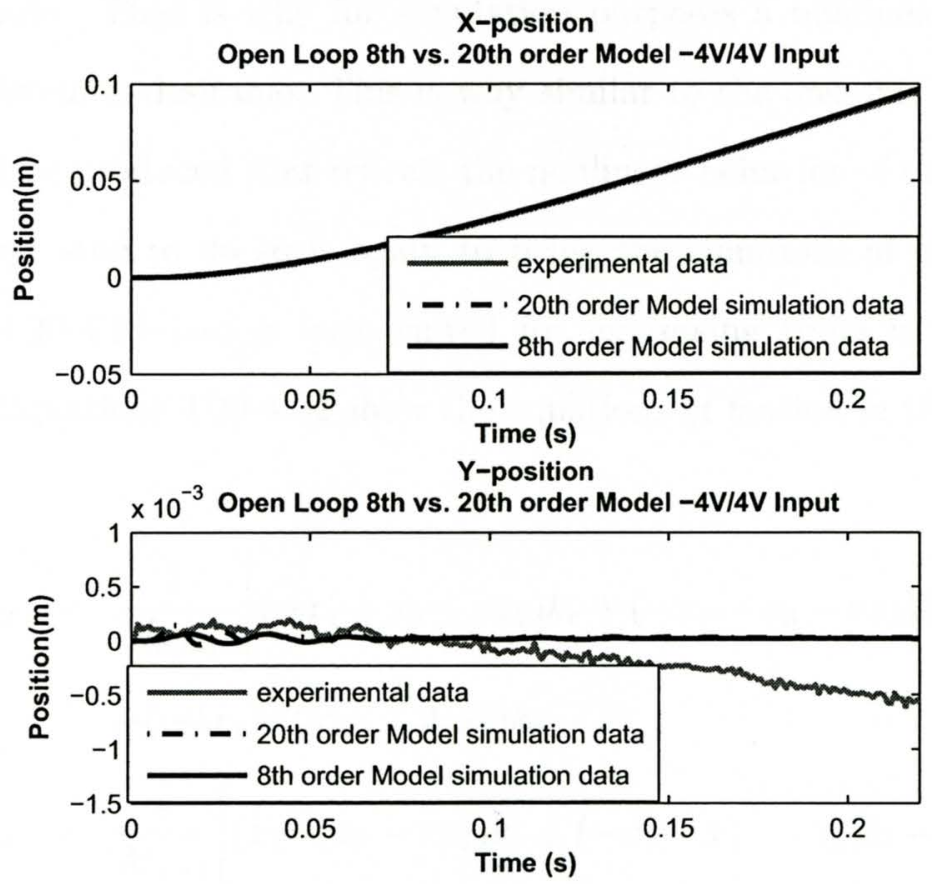

Figure 4.5. Open loop test $\mathrm{x}$-motion end-effector position 
assumptions made at the beginning of this chapter give a simplified lower order model, which approximates the prior 20th order model response very well and therefore as well reflects the dynamic behavior of the H-frame positioning system. Due to this conclusion and because of the advantages the lower order model has, the simplified model will be used for further work in this thesis.

In Figures 4.3 and 4.5 the same differences between experimental and simulation data as already pointed out in chapter 3 can be observed.

\subsection{Nonlinear Model of the H-frame System}

Similar to the one axis case, the $8^{\text {th }}$-order model is a strictly linear model and hence if those plots in Figures 4.2-4.5 would be redone for different input voltages with the same set of parameters shown in Table 4.1 it could be seen that the experimental and simulation data would not match as perfectly for those different voltage inputs. That is why for simulation purposes a nonlinear model for the $\mathrm{H}$-frame System is desirable. This is why similar to the one-axis case a Simulink model shall be produced that reflects the nonlinear behavior of the system.

The first step to do so is again to bring the equations of motion shown in equations 4.20-4.23 into a form suited for expressing them in Simulink block diagrams. Equations 4.29-4.32 show the equations of motion in that form.

$$
\begin{aligned}
\dot{x_{2}}= & \frac{1}{M_{\text {bridge }}}\left[\left(-x_{1}+x_{3}+r x_{7}\right) k_{r}+\left(-x_{1}-x_{3}-r x_{5}\right) k_{l}\right. \\
& \left.+\left(-4 x_{1}-2 r x_{5}+2 r x_{7}\right) k_{b}-F_{f_{x}}\right] \\
\dot{x_{4}}= & \frac{1}{M_{\text {cart }}}\left[\left(x_{1}-x_{3}-r x_{7}\right) k_{r}+\left(-x_{1}-x_{3}-r x_{5}\right) k_{l}-F_{f_{y}}\right] \\
\dot{x_{6}=} & \frac{1}{J_{M_{1}}}\left[\left(-r x_{1}-r x_{3}-r^{2} x_{5}\right) k_{l}+\left(-2 r x_{1}-r^{2} x_{5}+r^{2} x_{7}\right) k_{b}\right. \\
& \left.+\tau_{M_{1}}-\tau_{f_{M_{1}}}\right]
\end{aligned}
$$




$$
\begin{aligned}
\dot{x_{8}}= & \frac{1}{J_{M_{2}}}\left[\left(r x_{1}-r x_{3}-r^{2} x_{7}\right) k_{r}+\left(2 r x_{1}+r^{2} x_{5}+r^{2} x_{7}\right) k_{b}\right. \\
& \left.+\tau_{M_{2}}-\tau_{f_{M_{2}}}\right]
\end{aligned}
$$

Those 4 equations are now being expressed as block diagrams similar to that shown in the one axis case and stored in subsystems. They represent the two rotational motor axes, which have additional inputs $V_{i n_{1}}$ and $V_{i n_{2}}$ to compute the motor torques and look similar to the block diagram shown in Figure 2.19, and the 2 linear end-effector axes with their specific nonlinear frictions. The friction force in each of those axes is being computed with embedded Matlab functions with similar code as shown in Table 2.2. Those subsystems are then assembled to form the nonlinear H-frame model as shown in Figure 4.6.

Open loop simulations, that have been carried out for different input voltages, show the expected. The linear model matches the experimental data only for the amount of input voltage, for which it was tuned for $(-4 \mathrm{~V} /-4 \mathrm{~V}$ and $-4 \mathrm{~V} / 4 \mathrm{~V})$, however it deviates from the experimental responses for different inputs. The nonlinear model however is able to match also the different input voltages well. Table 4.2 shows the set of friction parameters, that were obtained to give those results.

The input Voltages for which the open loop simulation was carried out where ("Motor 1 input" /"Motor 2 input") -2V/-2V, -2V/2V, -4V/-4V, -4V/4V, -6V/-6V and $-6 \mathrm{~V} / 6 \mathrm{~V}$. This gives us 3 different motions in $x$-direction as well as 3 different motions in $y$-direction. Figures 4.7 through 4.18 show the results. 


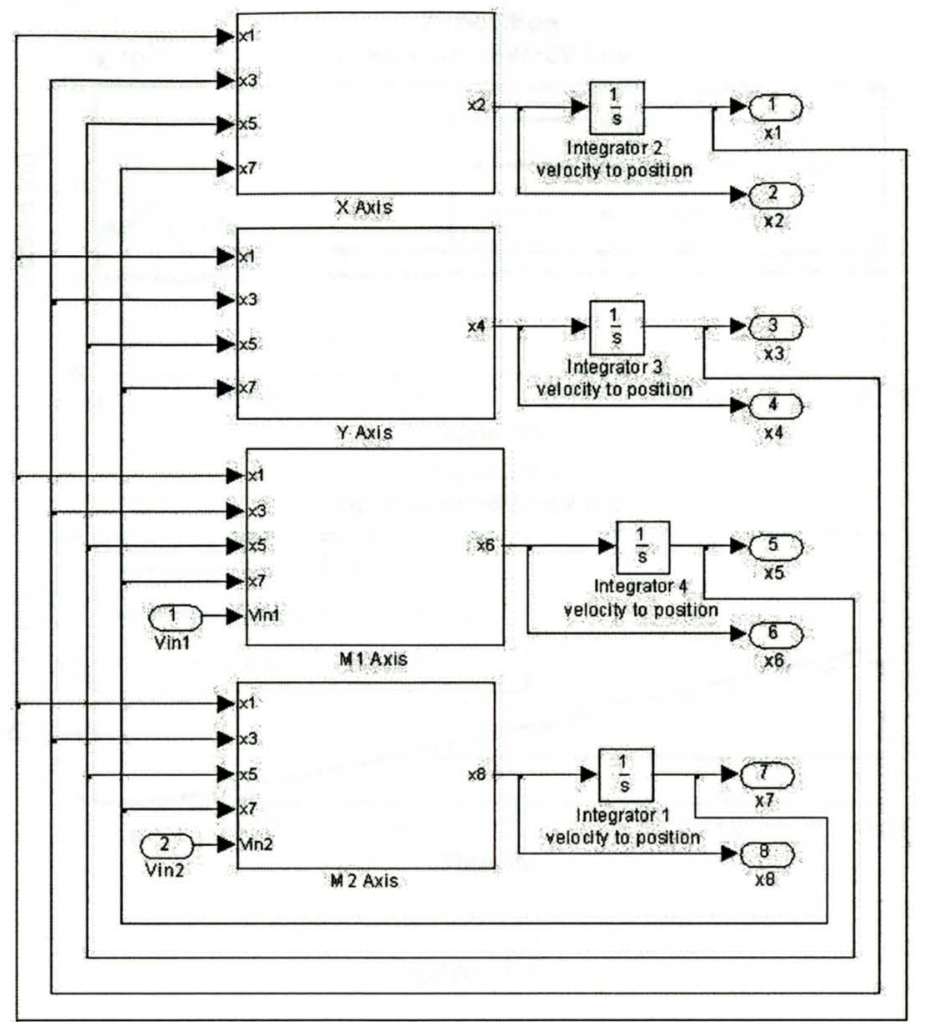

Figure 4.6. Nonlinear H-frame Simulink model

\begin{tabular}{|c|c|c|}
\hline & Variable & Value \\
\hline Viscous friction coefficients & $B_{M_{1}}$ & $0.0036 \mathrm{Nm} s$ \\
\cline { 2 - 3 } & $B_{M_{2}}$ & $0.0036 \mathrm{Nm} s$ \\
\cline { 2 - 3 } & $b_{\text {cart }}$ & $35.2944 \frac{\mathrm{Ns}}{\mathrm{m}}$ \\
\cline { 2 - 3 } & $b_{\text {bridge }}$ & $32.5114 \frac{\mathrm{Ns}}{\mathrm{m}}$ \\
\hline \multirow{7}{*}{ Coulomb force/torque } & $\tau_{c_{M_{1}}}$ & $0.05 \mathrm{Nm}$ \\
\cline { 2 - 3 } & $\tau_{\mathrm{c}_{2}}$ & $0.05 \mathrm{Nm}$ \\
\cline { 2 - 3 } & $F_{c_{\text {cart }}}$ & $0.4 \mathrm{~N}$ \\
\cline { 2 - 3 } & $F_{c_{\text {bridge }}}$ & $2 \mathrm{~N}$ \\
\hline
\end{tabular}

Table 4.2. Set of friction parameter $\mathrm{H}$-frame nonlinear model 

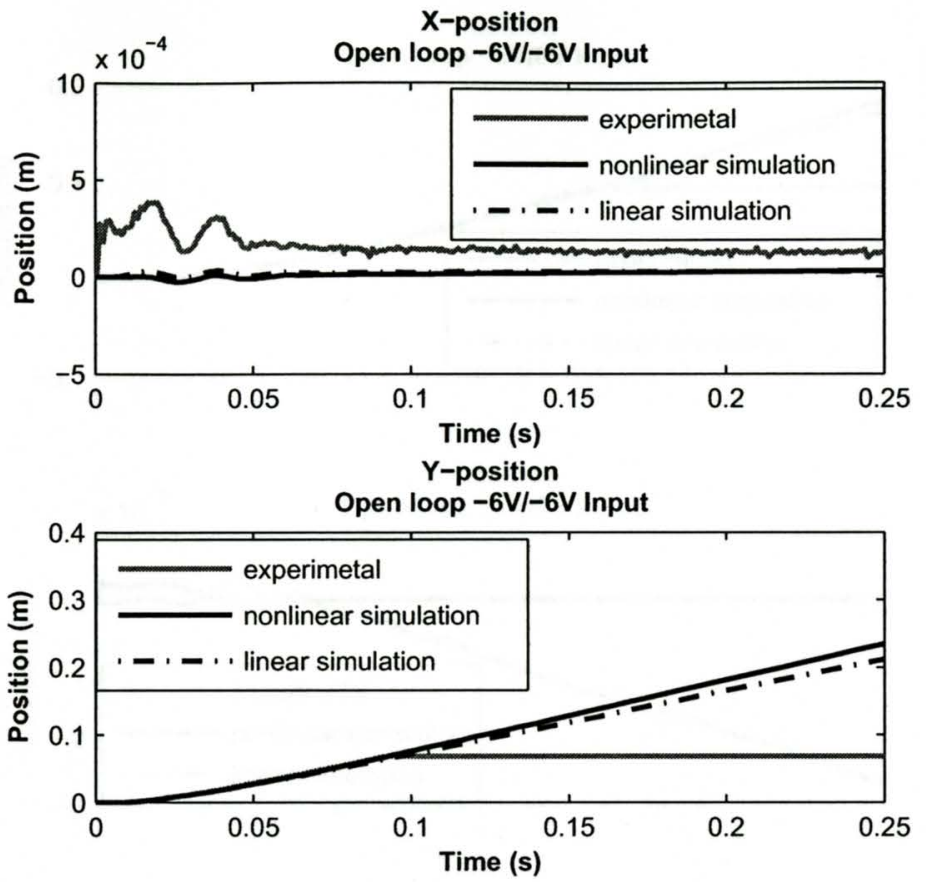

Figure 4.7.

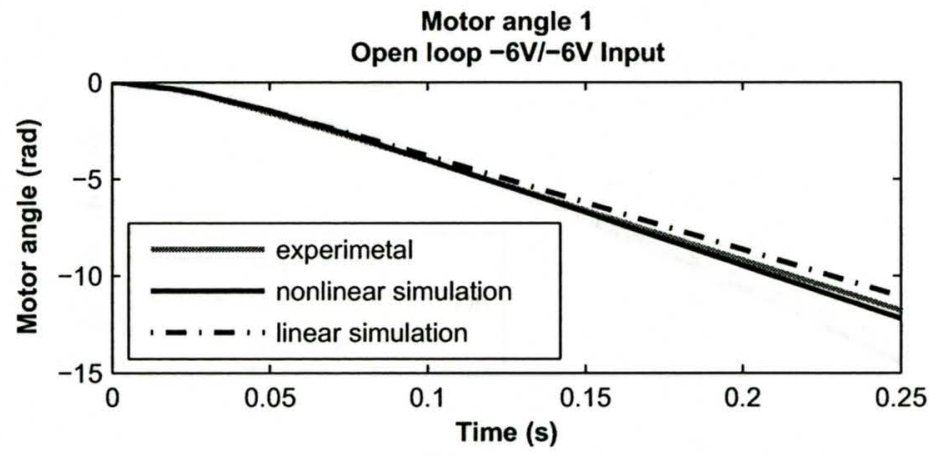

Motor angle 2

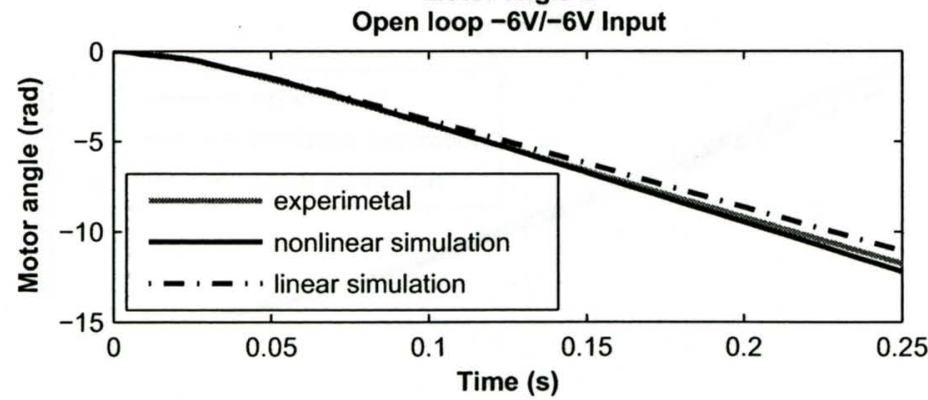

Figure 4.8. 

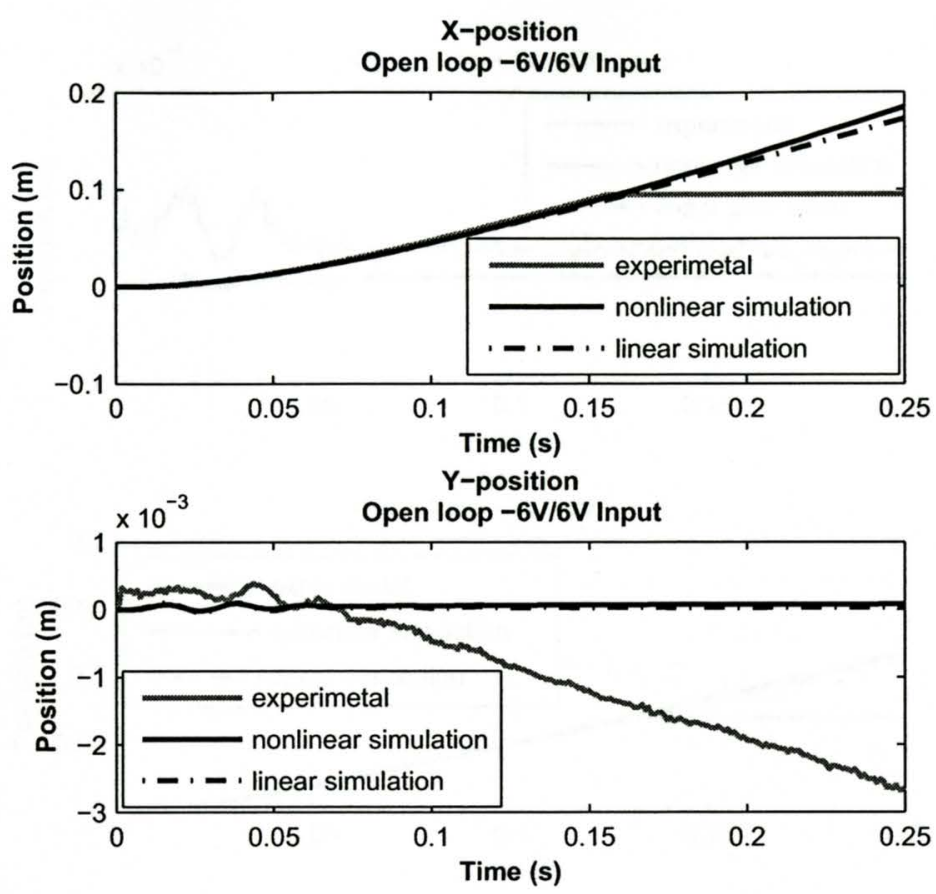

Figure 4.9.

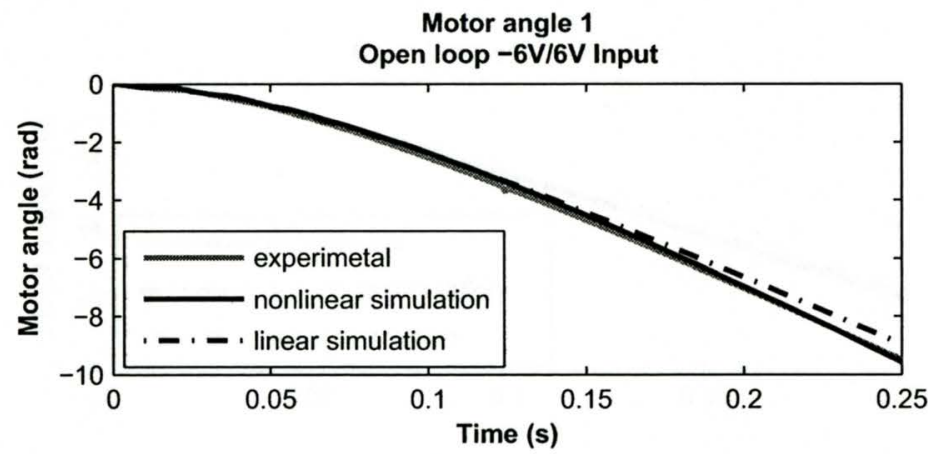

Motor angle 2

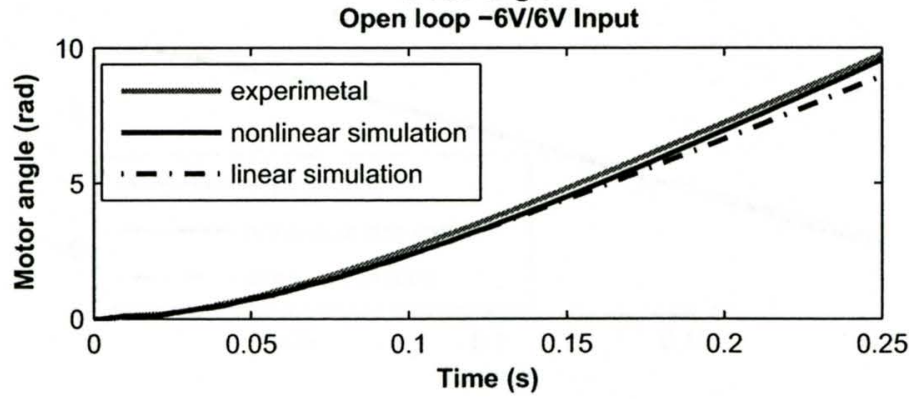

Figure 4.10. 
$X$-position
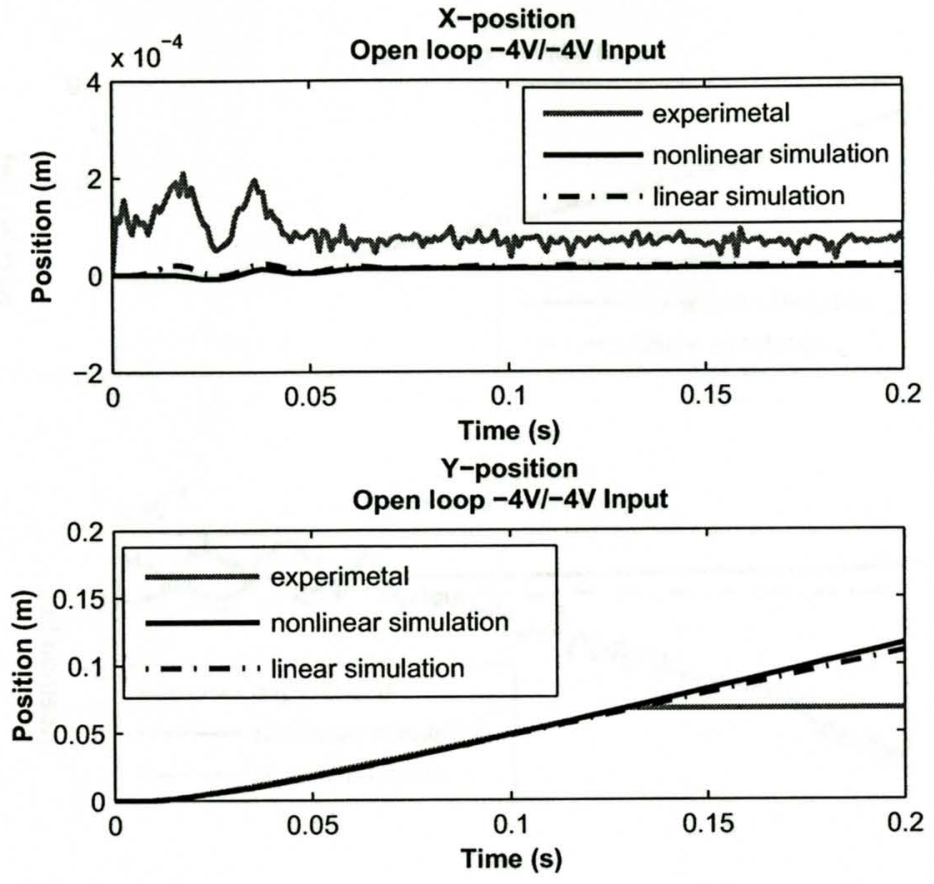

Figure 4.11.

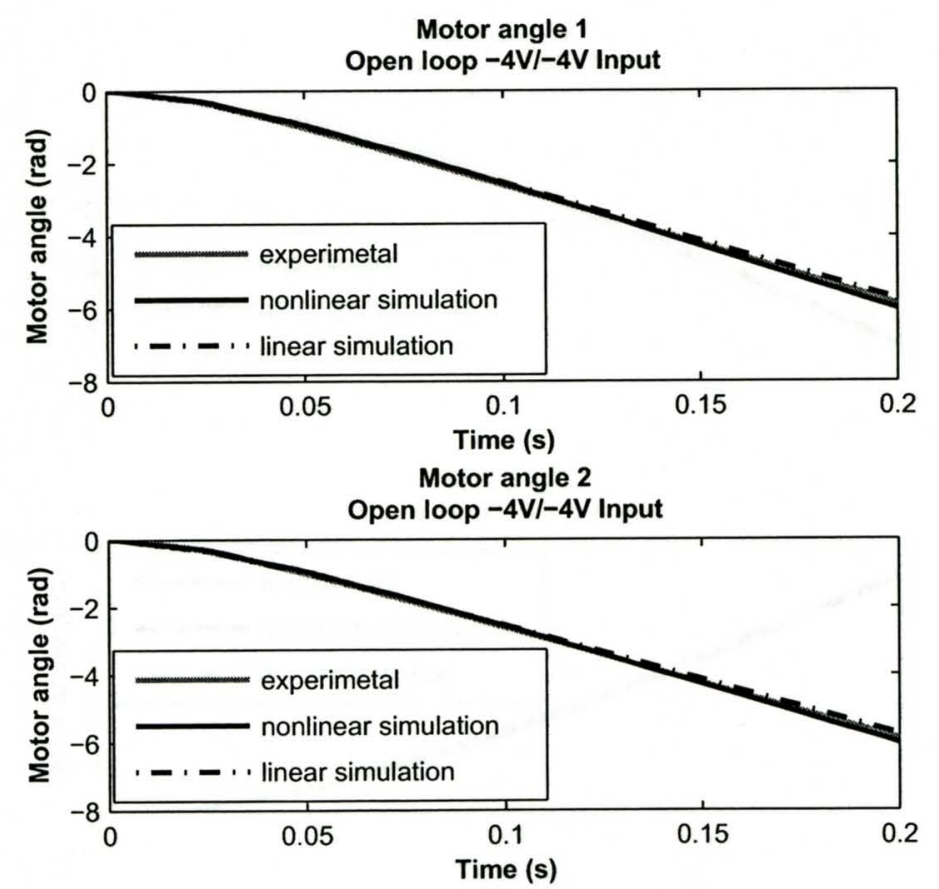

Figure 4.12. 

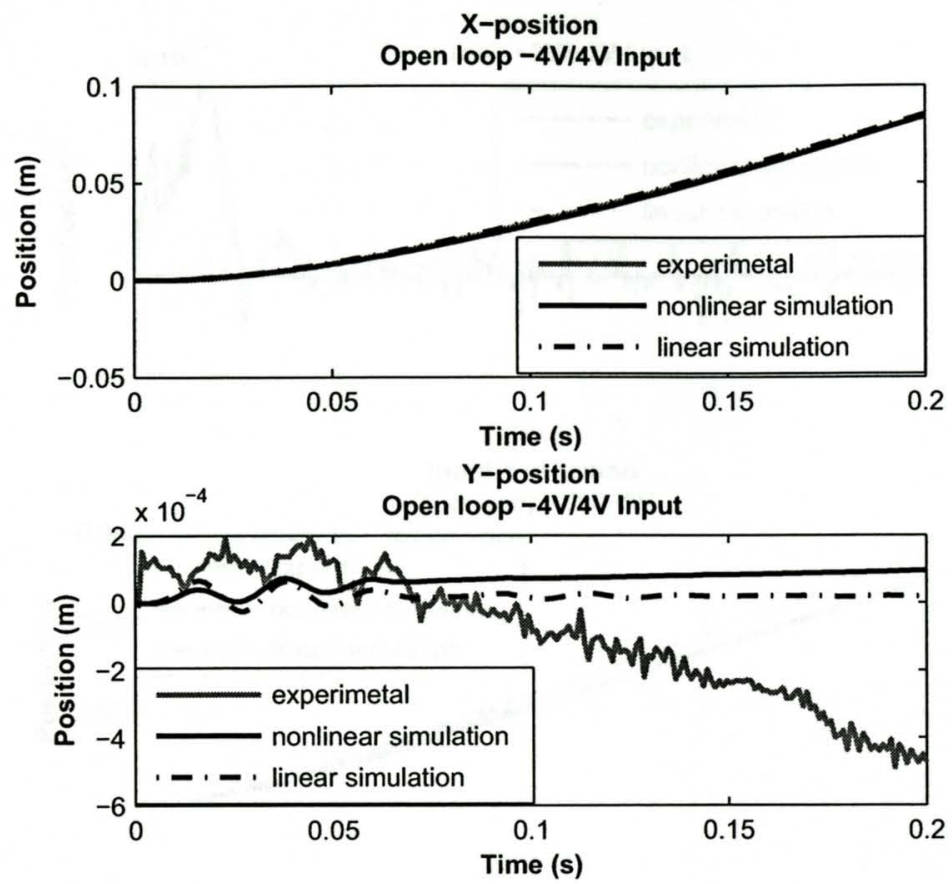

Figure 4.13.
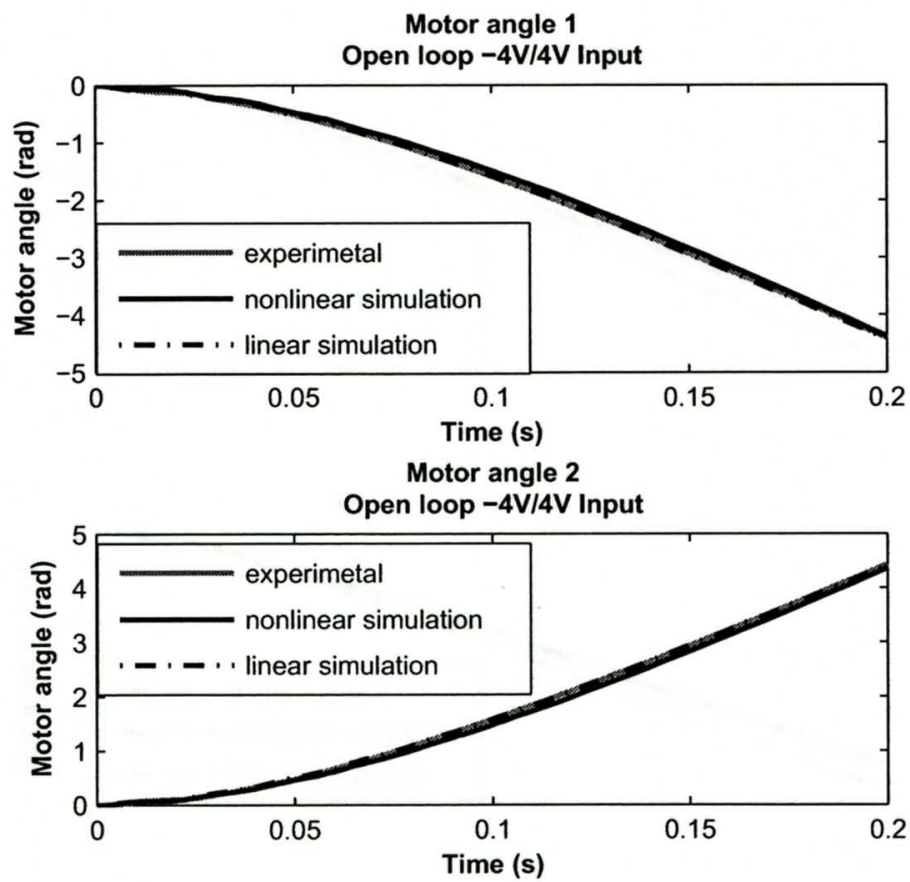

Figure 4.14. 

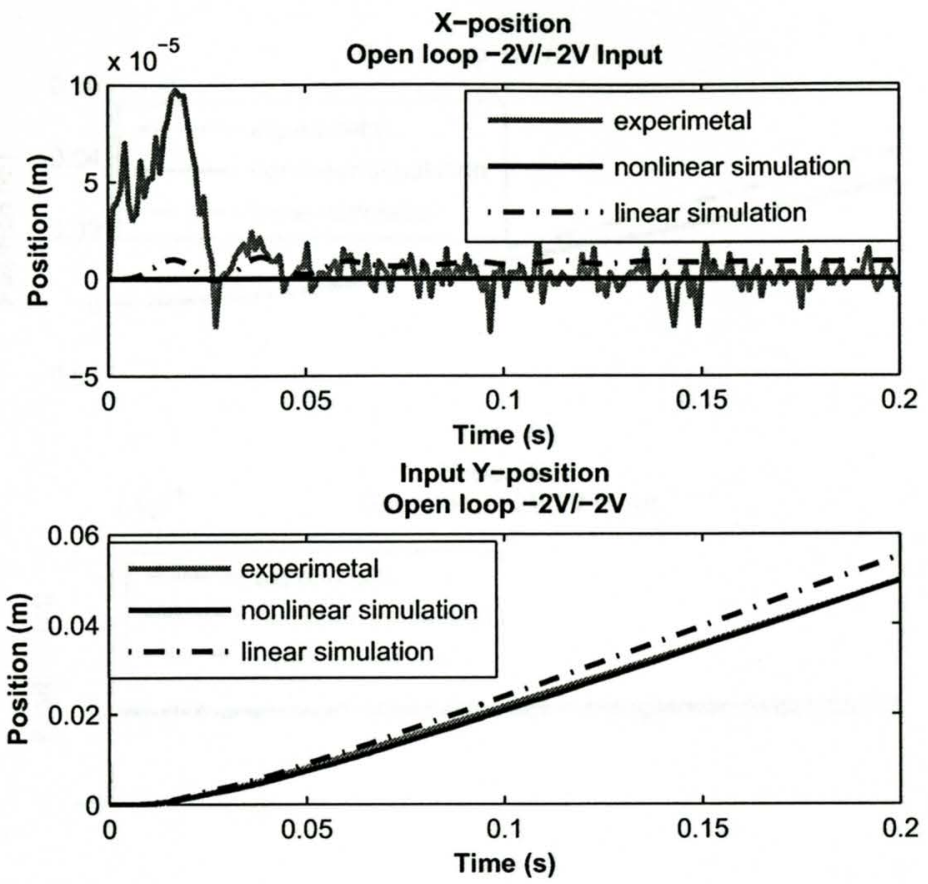

Figure 4.15.
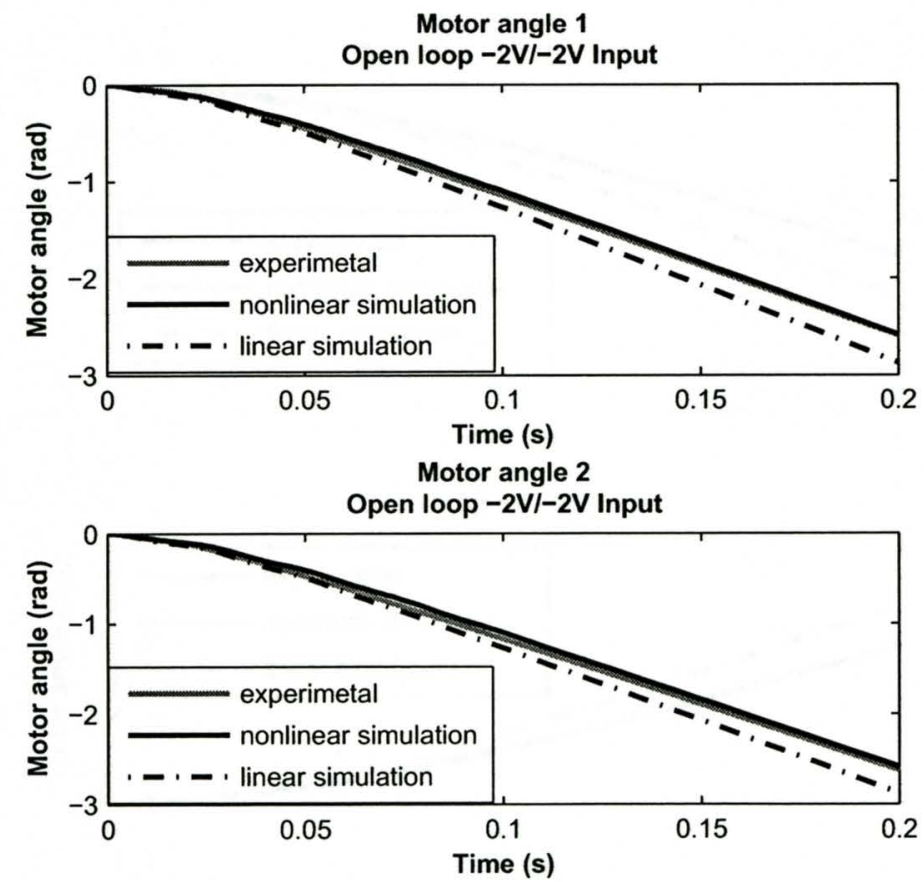

Figure 4.16. 

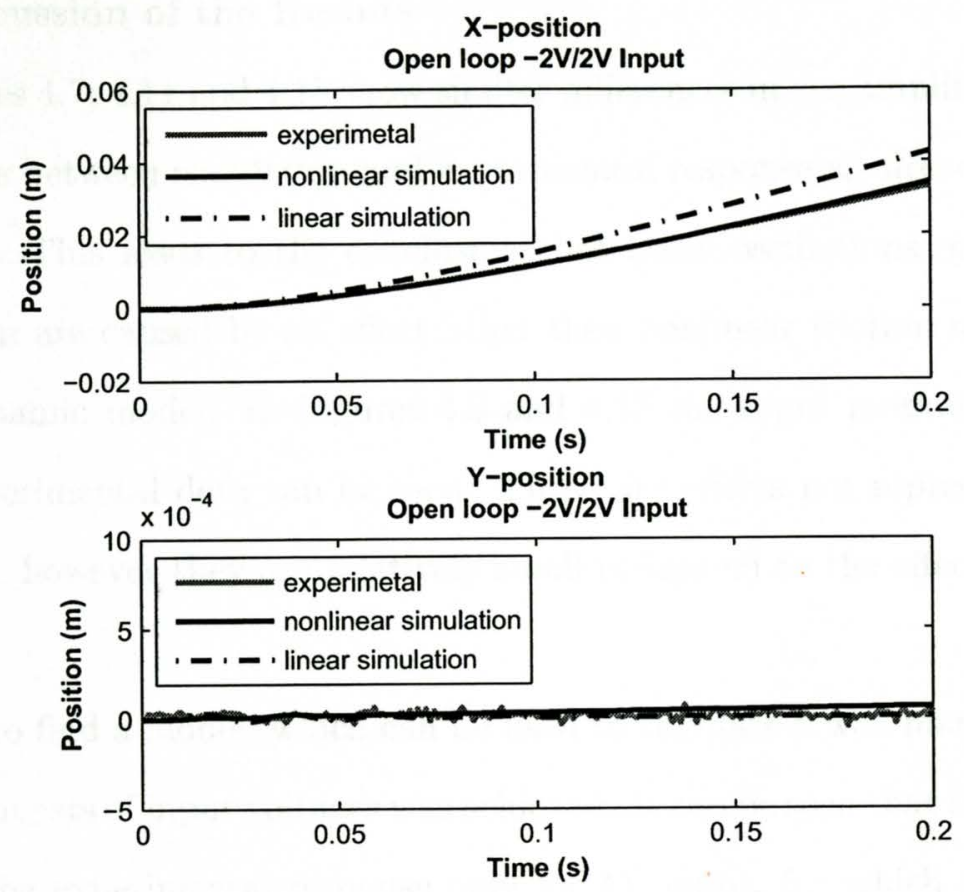

Figure 4.17.

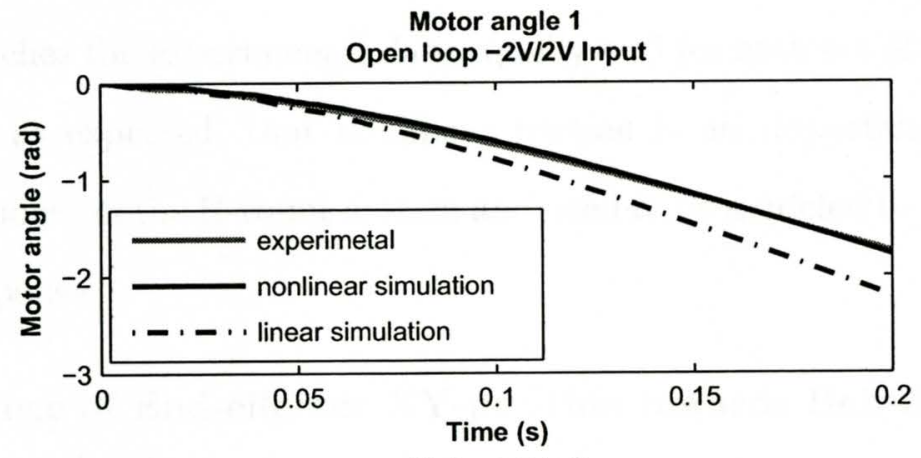

Motor angle 2

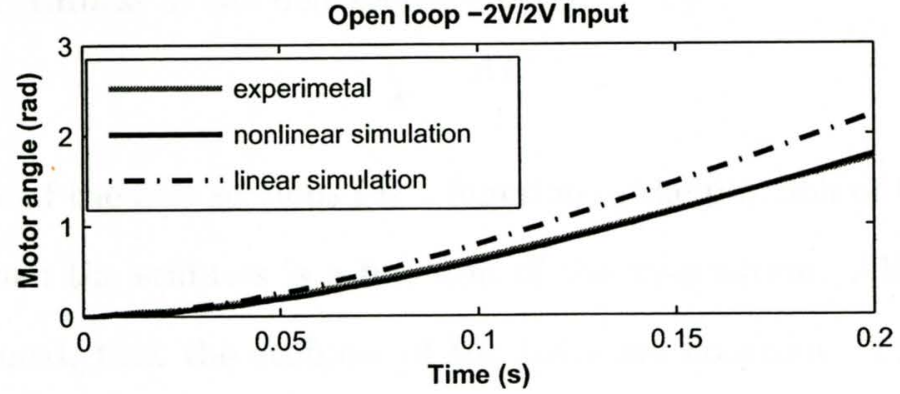

Figure 4.18. 


\subsection{Discussion of the Results}

Figures 4.7, 4.11 and 4.15 show similar differences in the amplitude of starting oscillations between simulation and experimental response as already discussed in chapter 3. This leads to the conclusion that those oscillations observed on the real system are caused by an effect other then nonlinear friction not represented in the dynamic model. In Figures 4.9 and 4.13 the slight motion in $y$-direction

of the experimental data can be seen. Those are effects not represented through the model, however they are relatively small compared to the effects of nonlinear friction.

The goal to find a model, which can be used to simulate a well matching response for different sets of input voltages was achieved. It can be seen that the linear model matches the experimental response only for $4 \mathrm{~V}$ input, for which the parameters were verified. However for $2 \mathrm{~V}$ and $6 \mathrm{~V}$ input the linear simulation clearly deviates from the experimental results. Where as the simulation of the nonlinear model in contrast matches the experimental data equally well for each set of input voltages. This shows, as expected, that nonlinear friction is an important factor in the dynamic response of the $\mathrm{H}$-frame system and need to be modeled to get an accurate dynamic response.

\subsection{Influence of End-effector XY-position towards Belt Stiffness}

Since the stiffness of the belt sections are given by

$$
k=\frac{A E}{l}
$$

and the length of the belt sections $l$ is a function of the position of the end-effector in $x$ and $y$, then the stiffness is a function of the xy-position. All simulations so far have assumed, that the stiffness of the belts are constant. Therefore in this section we study the effect of this change in stiffness on the dynamic behavior of the system. 
The first step to do so is to quantify the dependents of every single stiffness $\left(k_{1}\right.$ through $k_{9}$ ) on $x$ and $y$. The stiffness $k_{1}$ through $k_{9}$ depend on $x$ - and $y$-position of the end-effector as described in the following equations. Where $A$ is the cross section of the belt without the teeth, as explained before, $E$ is the E-Modulus of the belt and $l_{1}$ through $l_{9}$ are the belt section length measured at 0 -position of the end-effector located in the very middle of the H-frame system.

\section{$\underline{\text { Stiffness constant with respect to } x \text { and } y}$}

$$
k_{i}=\frac{A E}{l_{i}} \quad \text { for } \quad i=1,2,9
$$

The lengths of the belt sections in between the left motor pulley and the left stationary corner pulley $\left(k_{1}\right)$, as well as the one in between the right motor pulley and the right stationary corner pulley $\left(k_{2}\right)$ and also the one along the bridge $\left(k_{9}\right.$, which is not attached to the cart, do not change while performing motion in $x$ and $y$-direction.

$\underline{\text { Stiffness changing only with respect to } x}$

$$
\begin{array}{lll}
k_{i}=\frac{A E}{l_{i}-x} & \text { for } & i=3,4 \\
k_{i}=\frac{A E}{l_{i}+x} & \text { for } & i=5,6
\end{array}
$$

The belt sections in between the stationary corner pulleys and the bridge $\left(k_{5}\right.$ and $k_{6}$ ) get longer through a positive motion in $\mathrm{x}$ direction. At the same time the belt sections between the motor pulleys and the bridge $\left(k_{3}\right.$ and $\left.k_{4}\right)$ get shorter through the same motion.

$\underline{\text { Stiffness changing only with respect to } y}$

$$
k_{7}=\frac{A E}{l_{7}+y}
$$




$$
k_{8}=\frac{A E}{l_{8}-y}
$$

Only dependent on a change in $y$ are those two belt sections along the bridge which are connected to the cart $\left(k_{7}\right.$ and $\left.k_{8}\right)$.

Those above stated equations can now be plugged into equations 4.6 through 4.8 in order to obtain the stiffness $k_{l}, k_{r}$ and $k_{b}$, which are represented in the simplified 8th order model of the H-frame system.

$$
\begin{aligned}
& k_{l}=\frac{k_{1} k_{5} k_{7}}{k_{1} k_{5}+k_{1} k_{7}+k_{7} k_{5}}=\frac{A E}{\left(l_{1}+l_{5}+l_{7}\right)+x+y} \\
& k_{r}=\frac{k_{2} k_{6} k_{8}}{k_{2} k_{6}+k_{2} k_{8}+k_{6} k_{8}}=\frac{A E}{\left(l_{2}+l_{6}+l_{8}\right)+x-y} \\
& k_{b}=\frac{k_{3} k_{4} k_{9}}{k_{3} k_{9}+k_{3} k_{4}+k_{4} k_{9}}=\frac{A E}{\left(l_{3}+l_{4}+l_{9}\right)-2 x}
\end{aligned}
$$

To show the change in stiffness due to the change in xy-position, Figure 4.19 and 4.20 show the stiffness where one of the coordinates, $x$ or $y$, is held constant and the other one is changed over the workspace of the H-frame system. The plots show that there is a notable change in stiffness when the end-effector is moving through the workspace. While moving in $y$-direction $k_{l}$ and $k_{r}$ change by a factor of about 1.5 over the whole workspace. For a travel through the entire workspace in $\mathrm{x}$-direction the change of $k_{b}$ is most significant. It is changed by a factor of about 3 . Those results imply that the change in stiffness as a function of xy-position cannot be neglected with regard to end-effector position. So that for a accurate control over the whole workspace this change in stiffness has to be kept in mind. However for small displacements, as explored in the next chapter (1in by 1in motion or $0.0254 \mathrm{~m}$ by $0.0254 \mathrm{~m}$ ), the effect is rather negligible. 


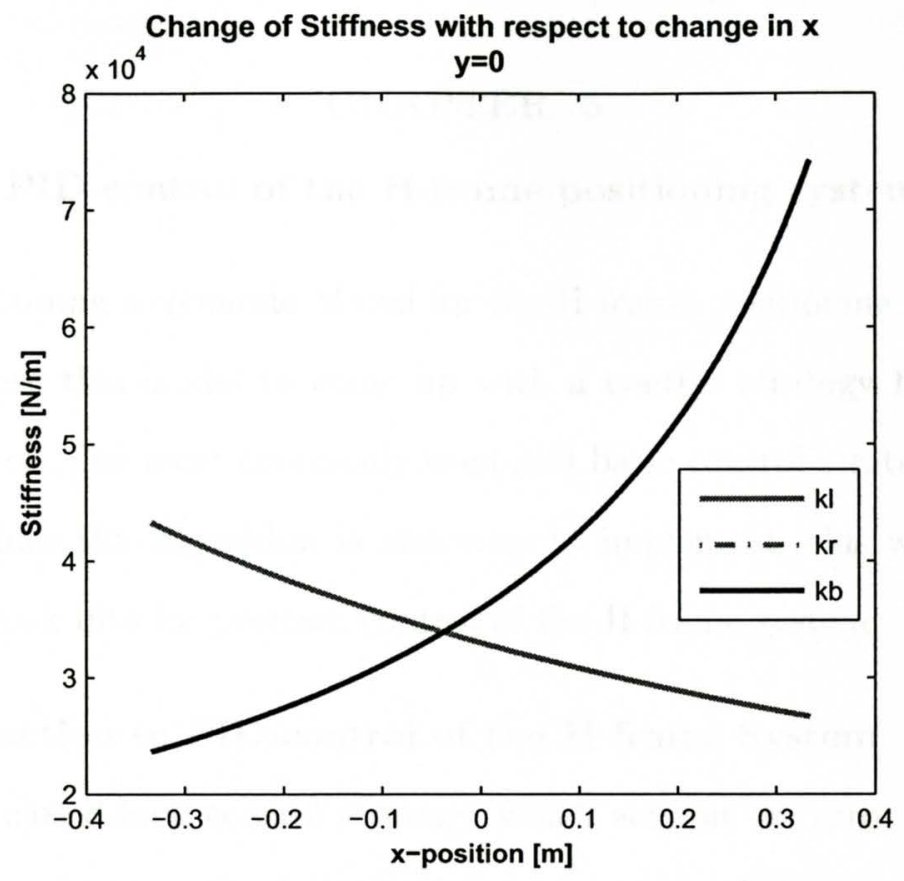

Figure 4.19. Change of Stiffness with respect to a change in $\mathrm{x}$

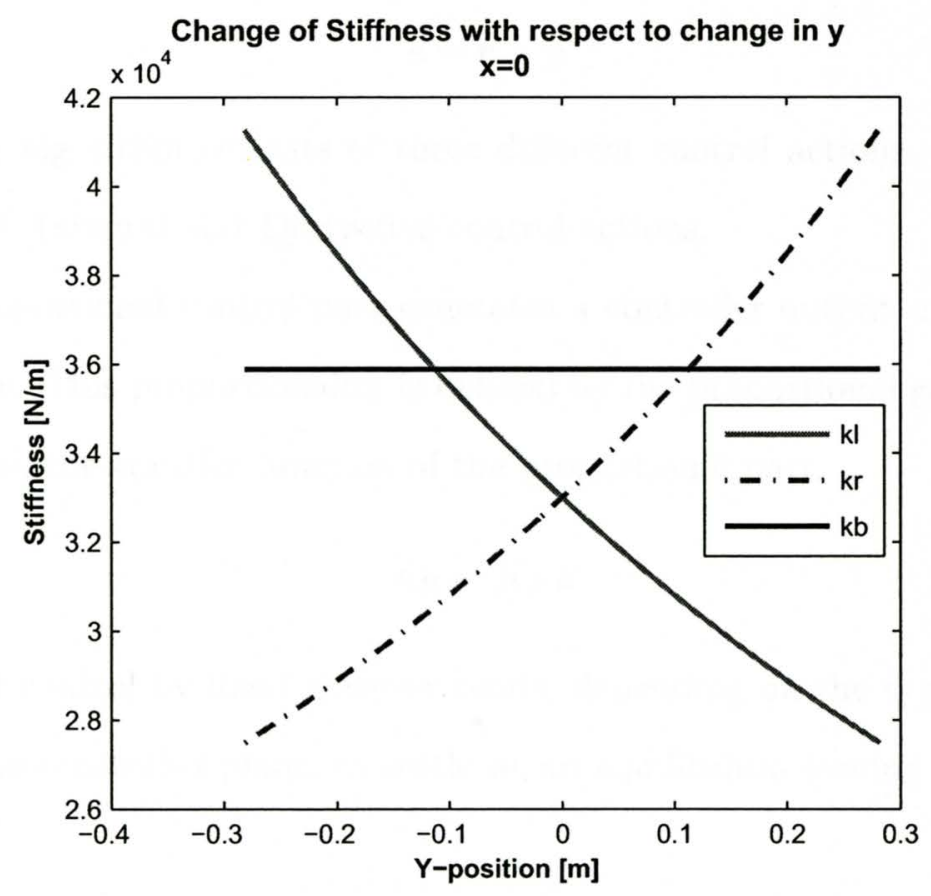

Figure 4.20. Change of Stiffness with respect to a change in y 


\section{CHAPTER 5}

\section{PID-control of the $\mathrm{H}$-frame positioning system}

After obtaining a dynamic Model for the $\mathrm{H}$-frame positioning system the objective is to use this model to come up with a control strategy for an accurate position control. The most commonly used and basic control strategy is the PIDalgorithm. Since this algorithm is also easy to implement, this will be the first controller to look into for position control of the $\mathrm{H}$-frame system.

\subsection{Introduction to PID-control of the $\mathrm{H}$-frame System}

PID is a closed loop control strategy, which acts on the error that is defined by the difference between the desired reference input $r$ and the actual plant output $y$.

$$
e=r-y
$$

The PID[10] algorithm consists of three different control actions. These are the

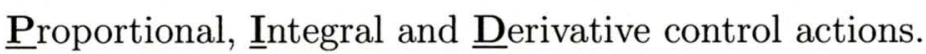

The Proportional control part generates a controller output $u_{P}$ proportional to the error $e$. This proportionality is defined by the proportional gain $K_{P}$. Equation 5.2 shows the transfer function of the proportional part.

$$
u_{P}=K_{P} e
$$

Proportional control by itself however tends, depending on the type of the input as well as the controlled plant, to settle at an equilibrium leaving a steady state error.

A way to possibly minimize the steady state error is to introduce integral control action. The integral part does not only act on the error, but it acts on the integral of the error and therefore on the error history. This error integral is being 
multiplied by the integral gain $K_{I}$ to obtain the integral part. In the time domain this is represented as:

$$
u_{I}(t)=\int_{0}^{t} e(t) d t \quad \text { for } \quad u(0)=0
$$

which translates into the s-domain transfer function shown in equation 5.4.

$$
u_{I}=K_{I} \frac{1}{s} e
$$

The integral part however has the potential to make the response overshoot and oscillate or even unstable, because the integral control output will increase until the error changes sign, which means an overshoot.

An oscillation can be prevented by introducing the third part of the PIDcontroller. The derivative part generates a control output proportional to the rate of change of the error. The proportionality is defined by the derivative gain $K_{D}$. In the time domain the derivative action is being expressed as

$$
u_{D}(t)=K_{D} \frac{d}{d t}(e)
$$

and in the s-domain, it is represented as

$$
u_{D}=K_{D} e s
$$

Note that a controller based only on the derivative action does not work since even a large but constant error would not generate any controller output. The derivative term has to be handled with special caution, since if implemented to a real system it can cause problems, because it amplifies measurerment noise which might occur in the system.

Those three control outputs $u_{P}, u_{I}$ and $u_{D}$ are summed up to get the PID control output $u$. Equation 5.7 shows the PID-controller transfer function.

$$
u=\left(K_{P}+K_{I} \frac{1}{s}+K_{D} s\right) e
$$




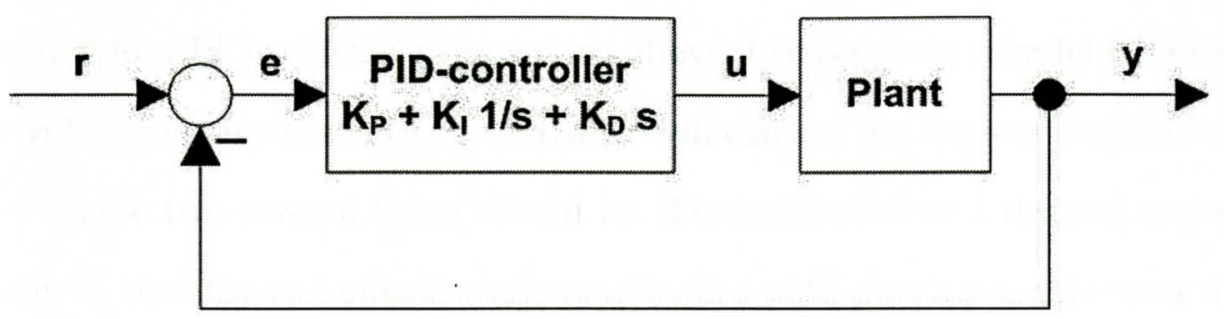

Figure 5.1. PID-control loop

Figure 5.1 shows the general setup for a PID-control loop.

The $\mathrm{H}$-frame positioning system is a multiple input multiple output (MIMO) system. The two voltages to the motors are the inputs and the $x$-position and the $y$-position are the outputs. The PID-control however is strictly for single input single output (SISO) systems. Thus the PID-implementation is not trivial. Furthermore the output that is desired to be controlled, the $x$ - and $y$-position, is not accessible as a feedback signal. This is because a measuring system able to accurately measure the $x y$-position over the whole workspace is expensive and therefore not economically. However the motor angles of the two motors are accessible as feedback signals. The motors are equipped with encoders, which measure the motor angles. Thus theoretically accurate control of the motor angles also controls the $x y$-position of the end-effector since the kinematic relation between motor angles and $x y$-position is known. This is of course only if the transmission elements between the motors and the end-effector are rigid. However since the belt is not perfectly rigid, an error in end-effector position is possible even if the motors are controlled perfectly. Nevertheless since the motor angles are the only possible feedback signals that indicate the output position and at the same time are economically available, those are chosen to be the feedback signals. To minimize the error occurring due to the nonrigid relationship between feedback signals and end-effector $x y$-position, which is the actual output that is desired to be controlled is one objective of a successful position control. Because PID-control 
is intended for SISO systems as explained above, two separate parallel PID-control loops will be implemented. Each controller will control the angular position of one motor. These two control loops should let the motors follow a desired trajectory perfectly so that the end-effector follows a desired path plus a possible error due to the stretch of the belt. Figure 5.2 shows the general setup of the two PID-control loops.

In the diagram $r_{M 1}, r_{M 2}, e_{M 1}, e_{M 2}, u_{M 1}, u_{M 2}, y_{M 1}$ and $y_{M 2}$ are the desired motor

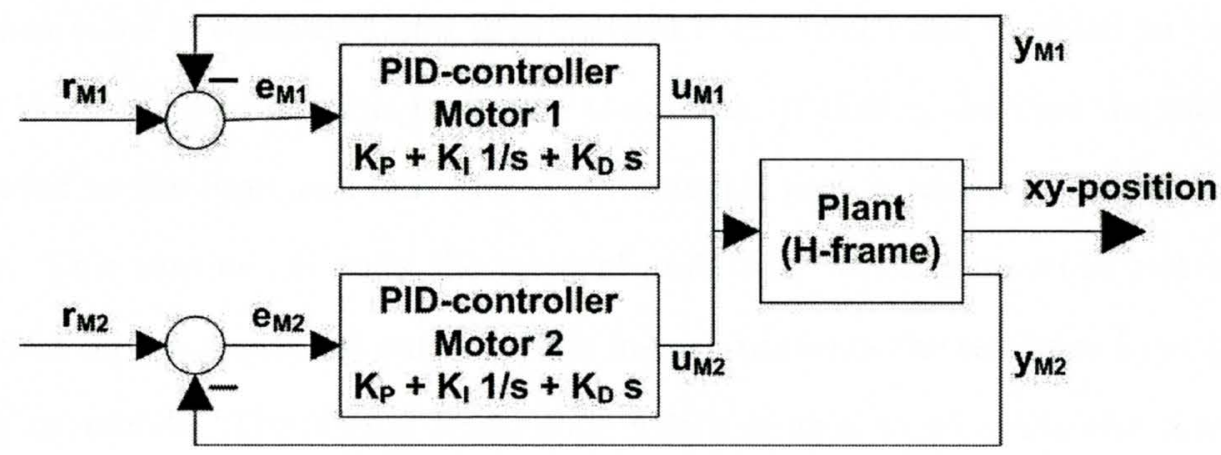

Figure 5.2. PID-control of the H-frame system

angle inputs for motor 1 and 2, the error in motor angle for motor 1 and 2, the controller outputs from the PID-controllers controlling motor 1 and 2 and the measured actual motor angles for motor 1 and 2 respectively. It also shows the setup with two separate parallel feedback loops for each motor. The desired output is the $x y$-position, however the measured and fed back signals are the motor angles.

The PID-controller used in the real system is a special kind called PIDcontroller with reset windup. This special routine accounts for the fact that even though in theory the controller output can be unbounded, however in real systems natural upper and lower bounds to the controller output are likely to exist, which bound the controller output inside a certain range. For the H-frame system those natural bounds are the D/A-converter output limits, which can only provide a maximum of $10 \mathrm{~V}$ and a minimum of $-10 \mathrm{~V}$ output. 
The PID-controller with reset windup logic first computes only the sum of the proportional and the derivative control outputs. It then checks if this already exceeds the upper or lower possible control outputs. If it does, the code does not calculate the integral control output at all and sets the output to the maximum or minimum output value depending on the case. More importantly it does also not add the current error to the error integral. If the controller output of the proportional and the derivative part does not exceed the maximum or minimum then first a trial integral value is computed and it is checked if the trial value if added to the rest does bring the overall value to exceed the limits. If that is the case the output is bounded to the limit and only the active integral part is added to the sum of the error. This routine prevents the integral part from winding up while not having an effect on the controller output. This in turn prevents the response from having a big overshoot. The Visual Basic code which is used to compute the controller output based on the above stated ideas is given in the Appendix A.

\section{$5.2 \quad$ Test trajectory}

To get comparable results and therefore to be able to make a statement of the accuracy the position control as well compare different strategies with each other a fixed test trajectory has to be agreed on. This test trajectory was chosen to be a 1in by 1 in $(0.0254 \mathrm{~m}$ by $0.0254 \mathrm{~m})$ square. To make the case more realistic the legs of the square are not being driven through with a constant speed profile, which would result in a displacement profile as shown in Figure 5.3, since this is not being done in practice either. Velocity profile like that would cause major overshoots on each corner, because a deceleration and acceleration is not intended.

Therefore for better corner tracking it is common to use a velocity profile which allows a certain acceleration at the start and deceleration towards the end position. It is chosen to have a constant acceleration and deceleration at the beginning and 


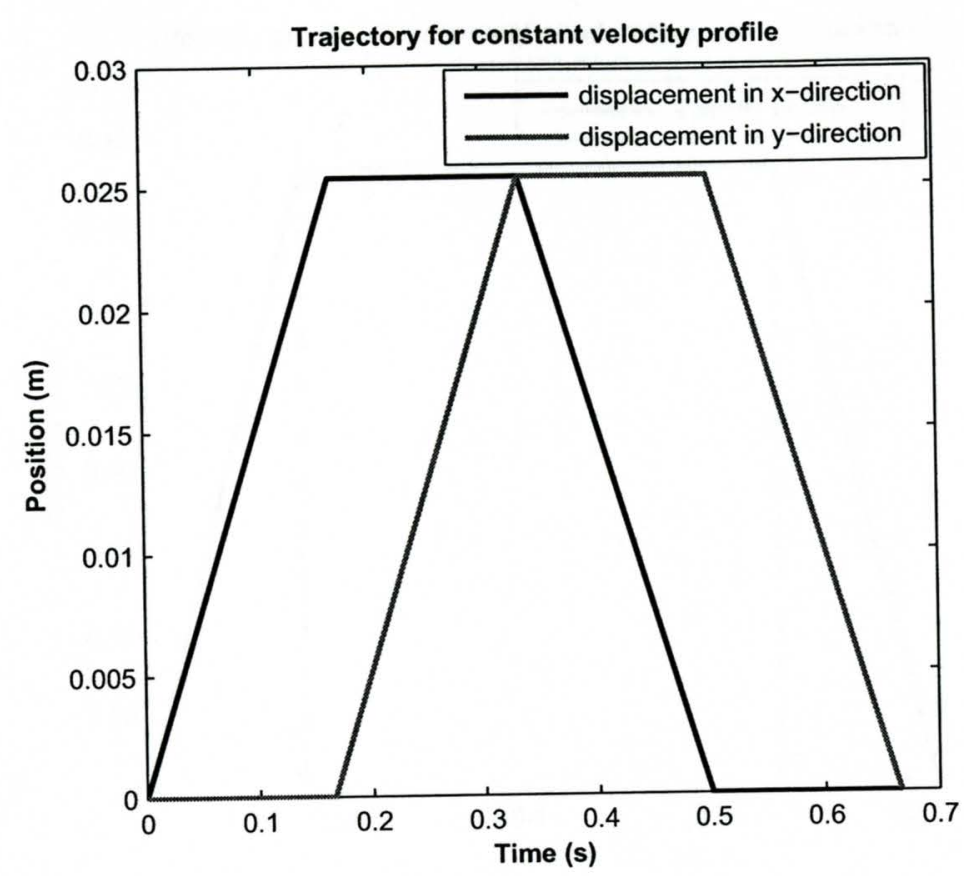

Figure 5.3.

the end of each one of the legs. Let the acceleration time $T_{a}$ be equal to the deceleration time $T_{d}=T_{a}$. Also let $f_{a}$ be a factor which relates the acceleration time $T_{a}$ to the overall time $T$ that is needed to run through the whole leg. So that

$$
T_{a}=f_{a} T
$$

For the test trajectory $f_{a}$ is chosen to be 0.2 and the maximum velocity which is also the velocity of the constant travel portion $v_{\max }$ is chosen to be $6 \frac{\mathrm{in}}{\mathrm{s}}\left(0.1524 \frac{\mathrm{m}}{\mathrm{s}}\right)$. Figure 5.4 shows the velocity profile for one leg corresponding to a desired displacement of 1 in.

The velocity is defined by the following equations.

$$
\begin{array}{lll}
v=\frac{v_{\max }}{T_{a}} t & \text { for } & 0 \leq t<T_{a} \\
v=v_{\max } & \text { for } & T_{a} \leq t<T-T_{a} \\
v=v_{\max }-\frac{v_{\max }}{T_{a}} t & \text { for } & T-T_{a} \leq t \leq T
\end{array}
$$




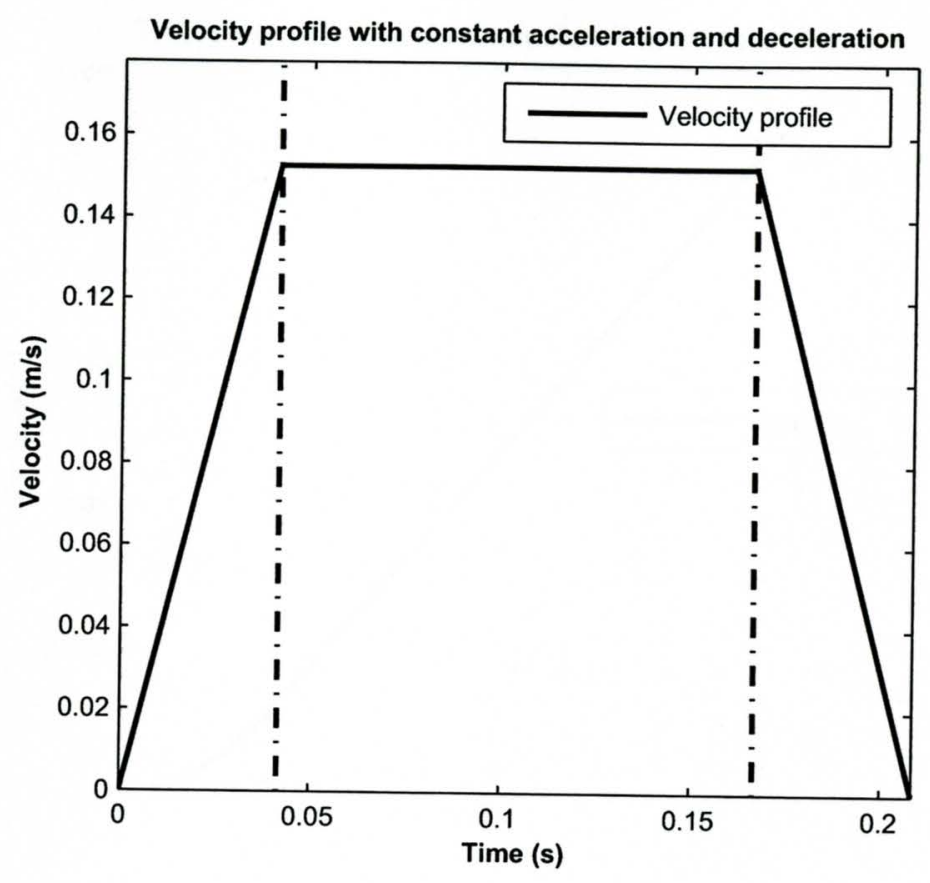

Figure 5.4. Velocity profile with constant acceleration and deceleration

In order to get the desired displacement those equations have to be integrated with respect to time. This gives the trajectory equations 5.10,

$$
\begin{array}{llll}
x= & x_{0}+\frac{1}{2} \frac{v_{\max }}{T_{a}} t^{2} & \text { for } \quad 0 \leq t<T_{a} \\
x= & x_{0}+\frac{1}{2} v_{\max } T_{a}+v_{\max }\left(t-T_{a}\right) & \text { for } \quad T_{a} \leq t<T-T_{a} \\
x= & x_{0}+\frac{1}{2} v_{\max } T_{a}+v_{\max }\left(T-2 T_{a}\right) & & \\
& +v_{\max }\left(t-\left(T-T_{a}\right)\right)-\frac{1}{2} \frac{v_{\max }}{T_{a}}(t-(T-T a))^{2} & \text { for } & T-T_{a} \leq t \leq T
\end{array}
$$

where $x_{0}$ is a a random starting position from where the motion is initiated.

Figure 5.5 shows the desired trajectory with constant acceleration and deceleration.

In the equations for the position there are three parameters $v_{m a x}, T$ and $T_{a}$. Since $T_{a}$ is related to $T$ through $f_{a}, v_{\max }$ is a parameter that we want to be able to chose, however instead of choosing the time $T$ that is needed for the motion we would rather be able to choose the distance $d$ to be traveled. That is why a relation between $T$ and $d$ is required. We can get it by setting the last one of the position 


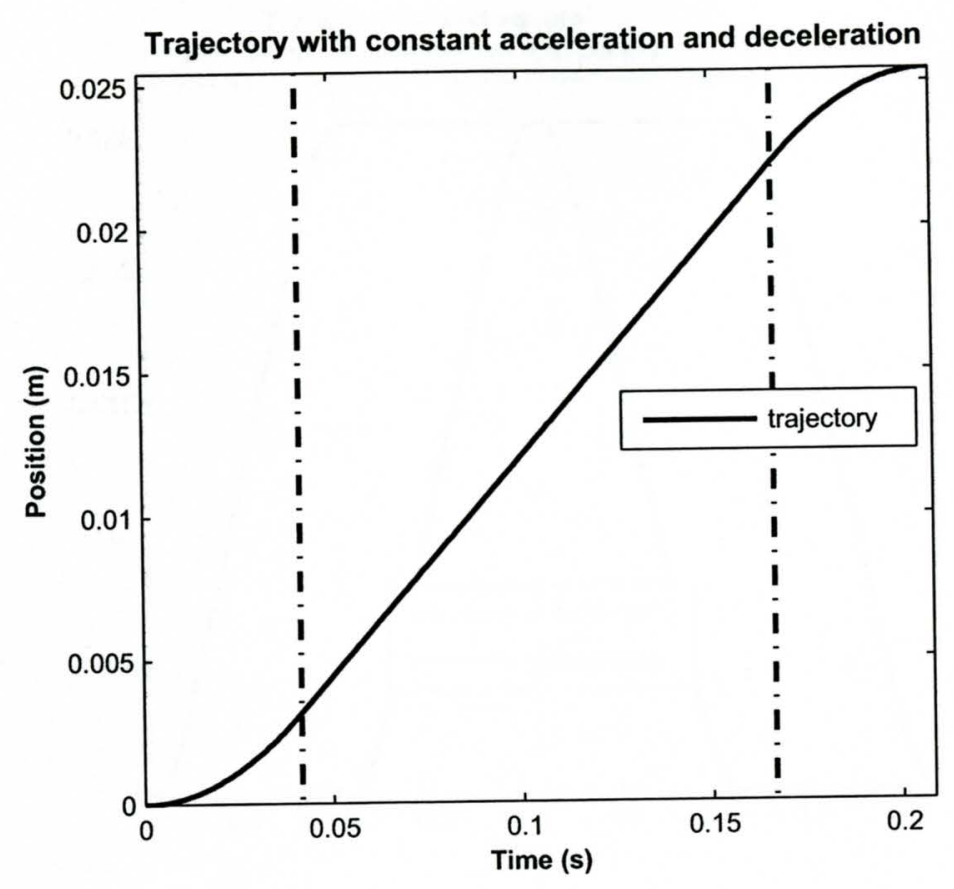

Figure 5.5. Trajectory with constant acceleration and deceleration

equations equal to $x_{0}+d$ at $t=T$. That gives us the following relation

$$
T=\frac{d}{v_{\max }\left(1-f_{a}\right)}
$$

and allows us to have three parameters, which can be chosen to uniquely determine the trajectory. Those parameters are $f_{a}, v_{\max }$ and $d$.

With those preliminary considerations its is easy to define a trajectory in $x$ and $y$ that follows our chosen 1 in by 1 in square with acceleration and deceleration on every leg. Figure 5.6 shows the desired end-effector trajectory.

Since the xy-position of the end-effector is not controlled directly however, this xy-trajectory has to be translated into desired motor angles for motor 1 and motor 2. This can easily been done with the kinematic relationship derived in chapter one between the motor angles and the xy-position of the end-effector.

$$
\left[\begin{array}{l}
x \\
y
\end{array}\right]=\left[\begin{array}{cc}
-\frac{1}{2} r & \frac{1}{2} r \\
-\frac{1}{2} r & -\frac{1}{2} r
\end{array}\right]\left[\begin{array}{c}
\varphi_{1} \\
\varphi_{2}
\end{array}\right]
$$




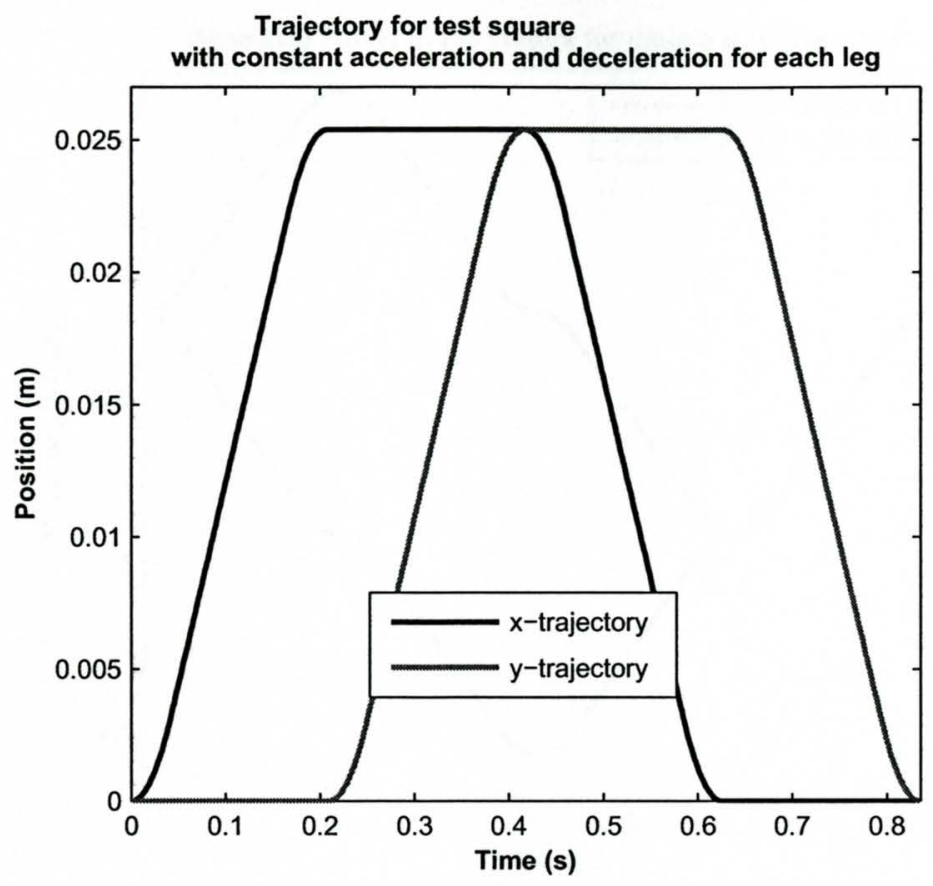

Figure 5.6.

This now gives us the desired reference inputs for our test square $r_{M 1}$ and $r_{M 2}$. Figure 5.7 shows the reference inputs versus time.

\subsection{PID simulation setup}

In order to find a PID setup which allows an accurate position control, the nonlinear model, derived in the previous chapter, will be utilized to first simulate the PID-control to understand the mechanisms and optimize the settings before implementing the control on the real system. Figure 5.8 shows the Simulink block diagram used to simulate the PID-control of the H-frame system.

The subsystem in the middle of the diagram contains the nonlinear model. The two embedded Matlab functions contain the code to generate the PID-controller output with the reset windup strategy; the code is very similar to the one shown in Appendix A, just translated into Matlab syntax. The zero order hold blocks in front and behind those embedded functions simulate the interface of the controlling 


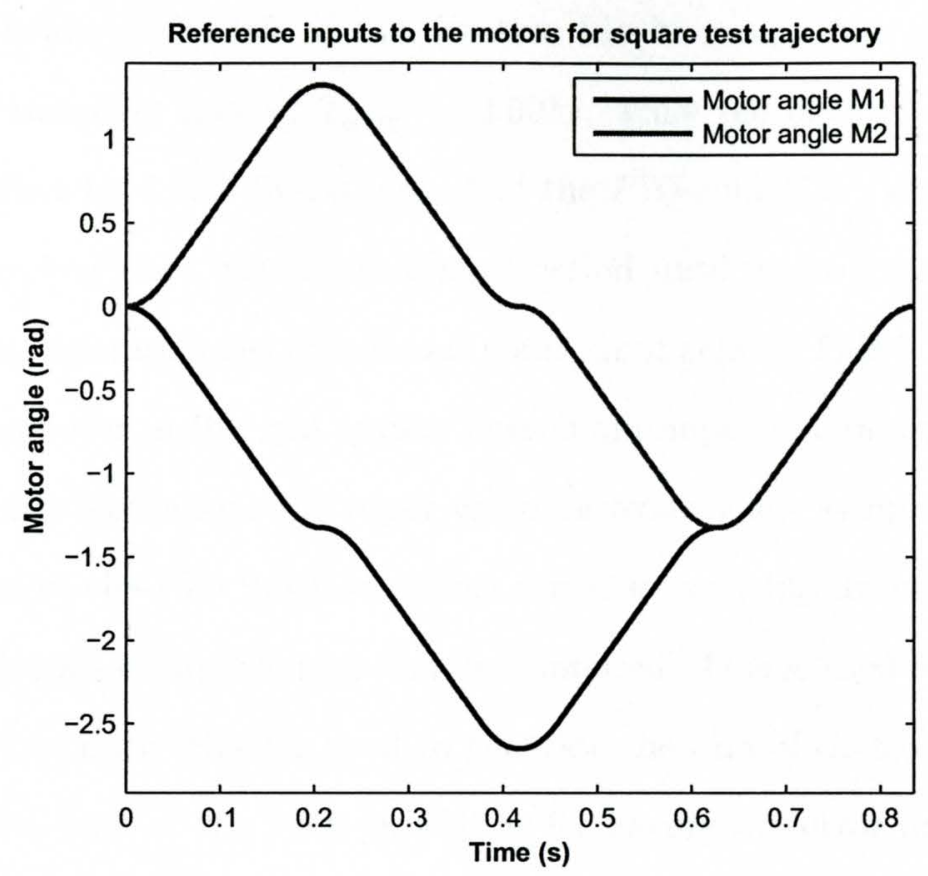

Figure 5.7.

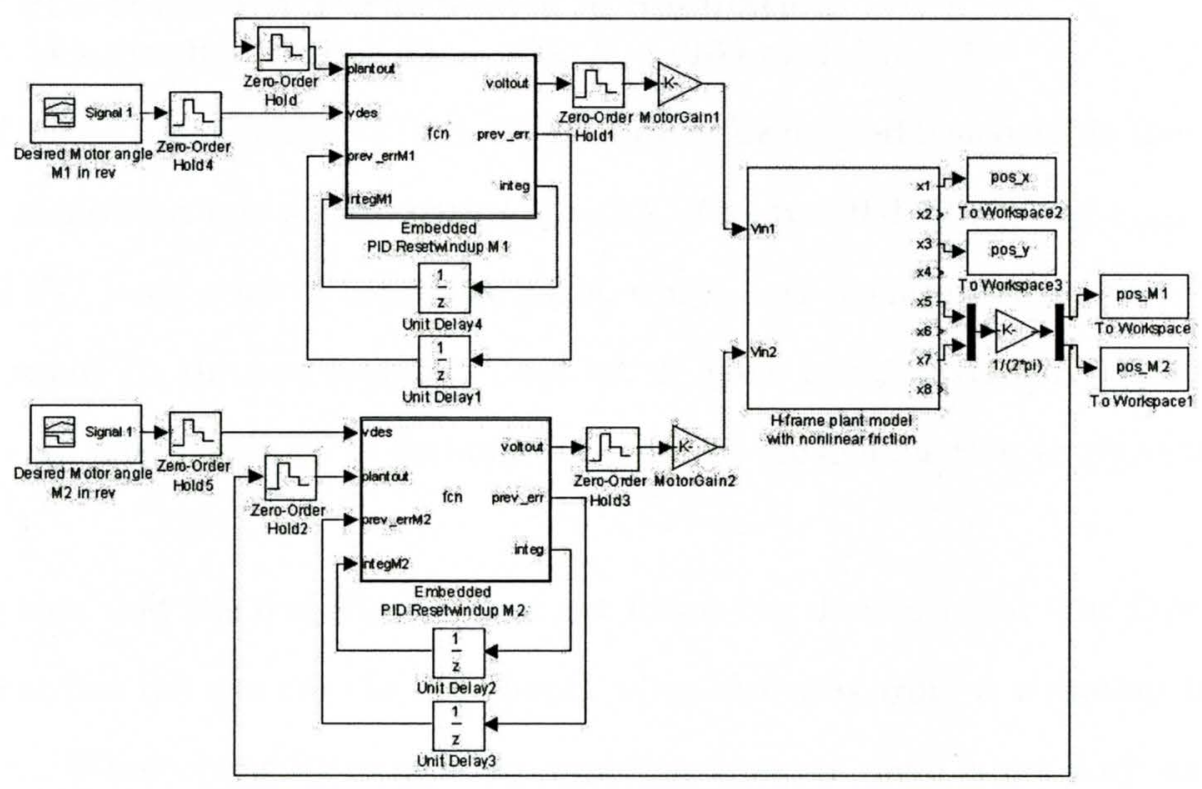

Figure 5.8. PID-controlled H-frame nonlinear model Simulink block diagram

digital personal computer and the continuous real world. The zero order hold blocks are equivalent to the $\mathrm{D} / \mathrm{A}$ - and $\mathrm{A} / \mathrm{D}$-converter in the real system. The 
zero order holds to the left of the function blocks sample the continuous input signal at a sampling time of $T_{\text {samp }}=0.001 \mathrm{~s}$, while the ones to the right of the PID-controllers hold the digital output of the PID-controller, which is evaluated at each sampling time, for one sampling period until a new controller output is computed to generate quasi continuous plant input signal. This is exactly how the Personal computer in the real system does it through its data acquisition board. The unit delay blocks save the input value for exactly one sampling period. This is been done so the PID function blocks can store variables in order to use them for their calculations in the next sampling interval. This is used for having access to the previous error which is used to generate the rate of change of the error for the derivative part of the PID, as well as for saving the error integral from one interval to the next.

\subsection{PID-controller Performance in Simulation}

\subsubsection{Controller Gains $K_{P}=150, K_{I}=100$ and $K_{D}=4$}

The first simulation of this PID-setup is being performed with the previously mentioned test square path $\left(f_{a}=0.2, d=1\right.$ in $(0.0254 m)$, and $v_{\max }=6 \frac{\mathrm{in}}{\mathrm{s}}$ $\left.\left(0.1524 \frac{\mathrm{m}}{\mathrm{s}}\right)\right)$ and a set of controller gains, which have been proven to give a fairly good result on the real system. That set of gains is $K_{P}=150, K_{I}=100$ and $K_{D}=4$. The resulting xy-end-effector-path for this simulation is given in Figure 5.9.

It is seen that the end-effector does not follow the desired path. The first thing that catches the eye are the overshoots when changing from a $x$-motion to a $y$ motion. When changing from $y$ - to $x$-motion however there is not such an overshoot. This makes sense since when moving in $x$-direction the whole bridge is being moved and has to be stopped, but when moving in $y$-direction only the light cart is being moved. The bridge due to its bigger mass has more inertia so it overshoots 


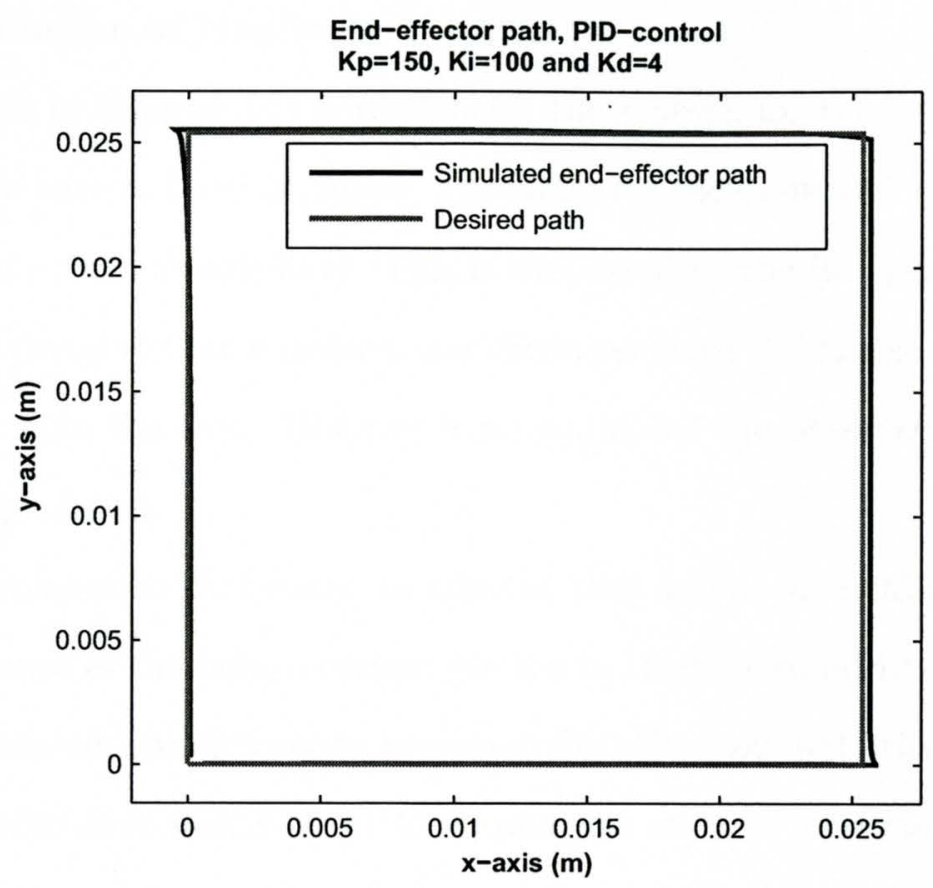

Figure 5.9.

more at the end of the leg.

Another thing that can be observed is the rather big offset in the steady state motion along the legs from the desired path in the $2^{\text {nd }}, 3^{\text {rd }}$ and $4^{\text {th }}$ leg.

Table 5.1 summarizes the deviation measured perpendicular to the desired path. Those characteristics will later be used to verify any improvement.

\begin{tabular}{|c|c|c|c|c|}
\hline & $1^{\text {st }}$ leg & $2^{\text {nd }}$ leg & $3^{\text {rd }}$ leg & $4^{\text {th }}$ leg \\
\hline $\begin{array}{c}\text { Overshoot } \\
\text { at the end of the leg } \\
\text { in } m\end{array}$ & $5.82 \times 10^{-4}$ & $\begin{array}{c}\text { Undershoot } \\
-1.74 \times 10^{-4}\end{array}$ & $5.02 \times 10^{-4}$ & $/$ \\
\hline $\begin{array}{c}\text { Offset } \\
|\max | \\
\text { in } m\end{array}$ & $3.35 \times 10^{-5}$ & $2.72 \times 10^{-4}$ & $1.15 \times 10^{-4}$ & $1.05 \times 10^{-4}$ \\
\hline
\end{tabular}

Table 5.1. Path deviations PID-control; $K_{p}=150, K_{I}=100$ and $K_{D}=4$ 


\subsubsection{Influence of Nonlinear Friction}

In order to improve the performance, the reasons for the deviation from the desired path have to be understood. The cause for the overshoot is easily identified as the inertia that the axis have. This is supported by the fact that there is only a substantial overshoot for a motion in $x$-direction since the bridge has significantly more mass than the cart. However a reasoning for the observed offset is not as trivially explainable.

The first explanation that comes to mind is, that due to the nonlinear friction and the compliance of the belt, a certain stretch in the belt could remain, which does not create enough spring force to overcome the offset coulomb friction of the linear axis. However that would mean if we plot the encoder position translated into $x y$-end-effector position with equation 5.12 , no offset should remain, but that is not the case. Figure 5.10 shows that plot.

It can bee seen that a rest stretch of the belt due to nonlinear friction is not the only explanation for the offset observed. To better be able to compare the error of the end-effector position a new plot is being introduced. Figure 5.11 shows the error of the end-effector from its desired value. However since for now only the actual deviation from the desired position, but not the lag in time is of interest, the plot shows for each leg only the perpendicularly measured error from the leg that is being driven through at that time. So in leg one only the deviation from the $y$-position is being shown, for leg 2 only the deviation from the $x$-position and so on. The plot shows the errors in $y$-direction for the simulated end-effector path laying above the desired path as positive and vise versa, for the error in $x$-direction a simulated path right of the desired path is shown as a positive and left of the desired path as a negative error. Figure 5.11 shows the end-effector error and the end-effector error based on the motor angles in this kind of plot. 


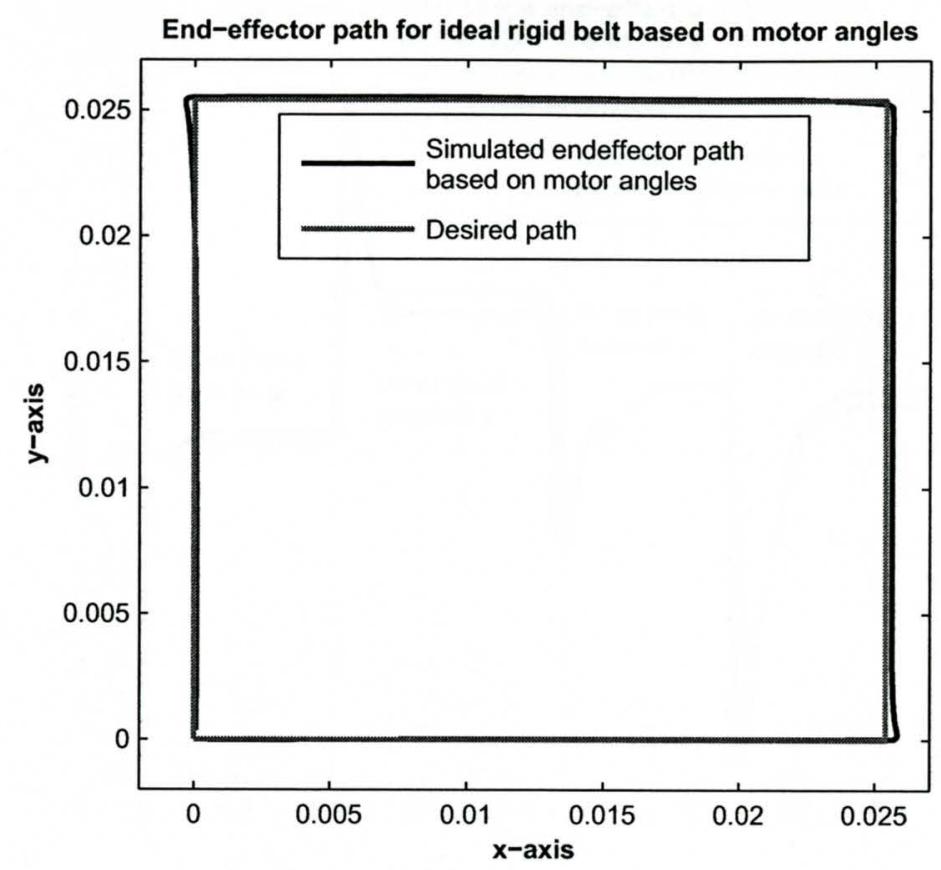

Figure 5.10.

This plot shows even clearer that even though the nonlinear friction combined with a rest stretch has some effect on the offset, however the biggest amount of this offset is caused by some other effect.

\subsubsection{Influence of Inequality in Motor Errors}

To investigate the cause for the offset, the actual controlled variables, the motor angles have to be examined more closely. Figure 5.12 shows the desired motor angles versus the actual motor angles.

The plots reveal, that the actual motor angles always lag behind the desired motor angles. This normally would not be a major factor, if the lag in time for both motors is equally the same, meaning that the absolute value of the errors are equal. Figure 5.13 shows the difference between actual motor angle and desired motor angle at any time for each one of the motors and the figure shows that the absolute errors for both motors are not equal. 


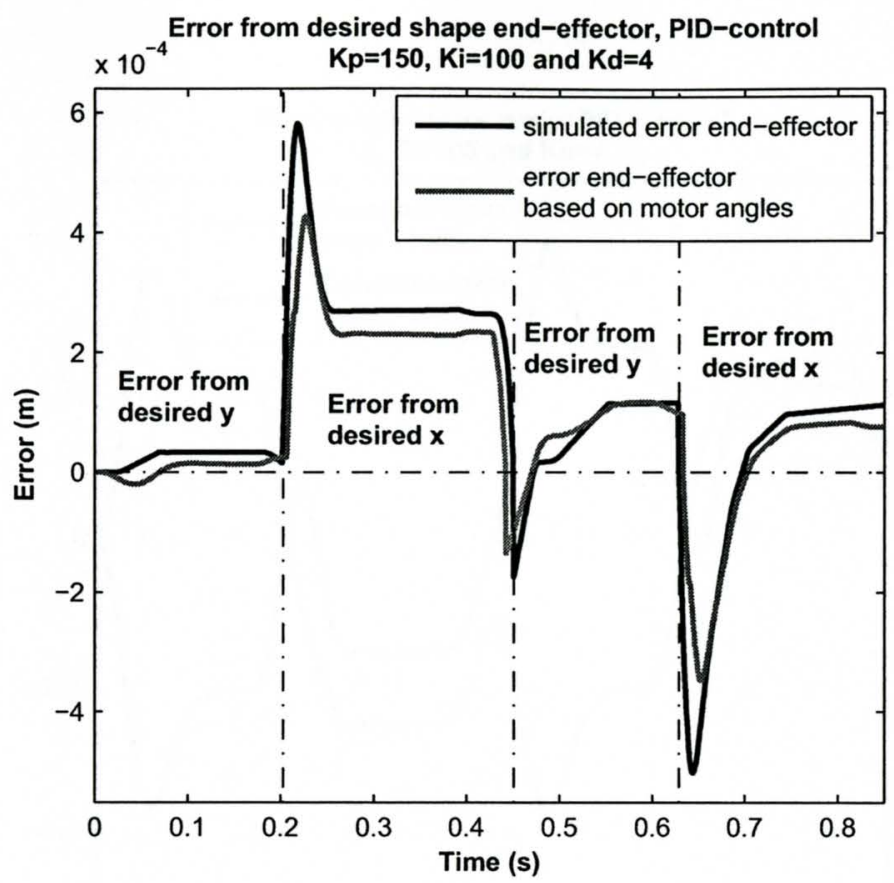

Figure 5.11.

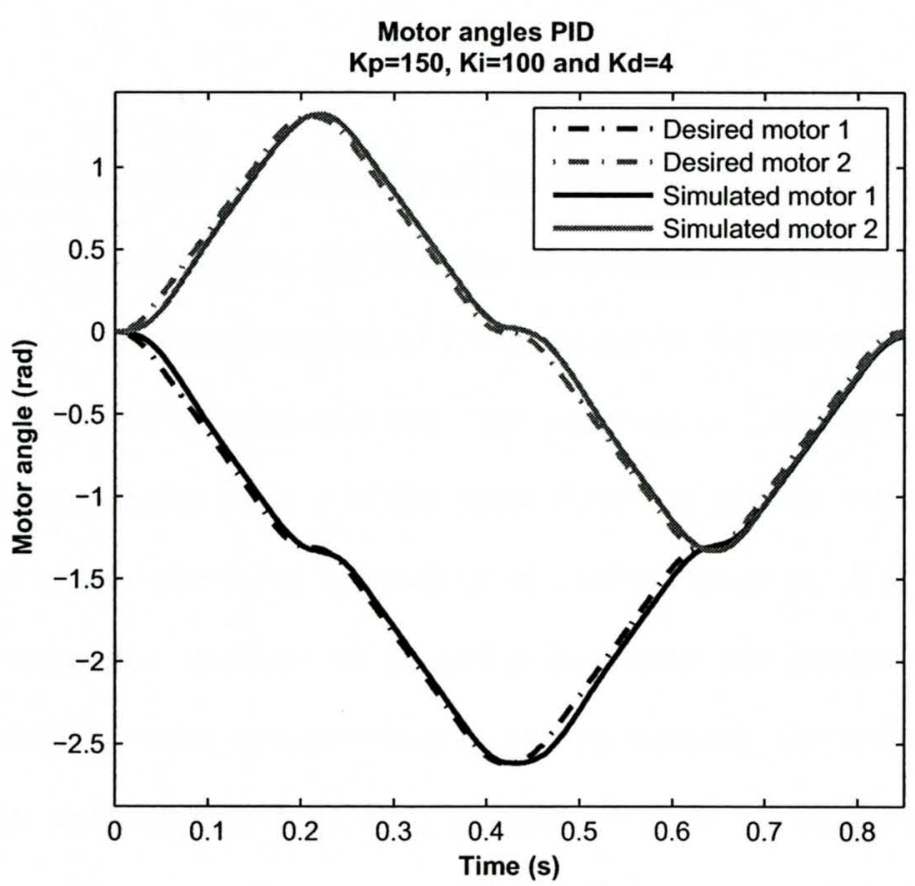

Figure 5.12. 


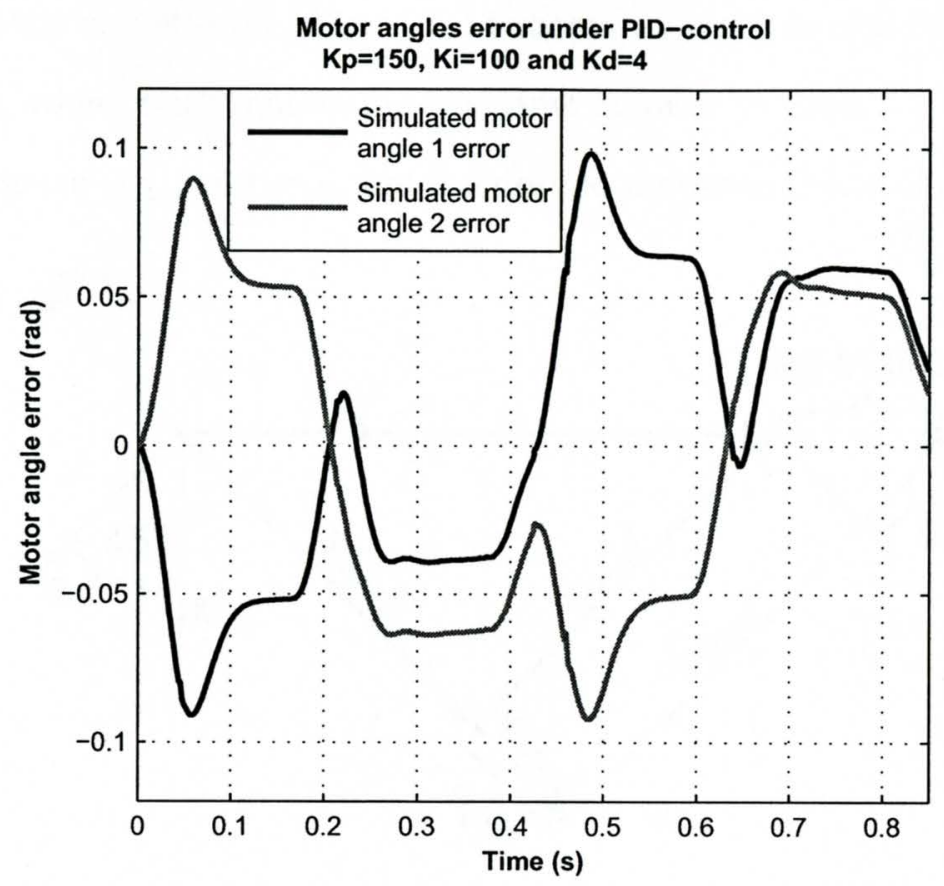

Figure 5.13.

To understand how an inequality of the motor angle errors causes the offset, a closer look at the kinematic of the $\mathrm{H}$-frame system has to be taken. As explained in the first chapter, the superposition of the rotation of the two motors is causing the motion in $x$ and $y$ of the end-effector. An $y$-motion is generated by a rotation of the same amount of the motors in the same direction and an $x$-motion is obtained if the motors rotate the same amount in opposite direction. If the motors rotate by different amounts, motions in $x$ - and $y$-directions are generated. To visualize the relationship between motor rotation and $x y$-motion, the motor angles can be visualized, by multiplying them by the radius $r$ as a set of linear perpendicular coordinates positioned at the same origin as the linear $x y$-coordinates, but mirrored along $x$ and rotated negative $45^{\circ}$, as shown in Figure 5.14. In those set of coordinate we can now plot the linear displacement due to each motor rotation 
as two separate vectors. The vector sum of those vectors is giving the according position of the end-effector. Figure 5.14 shows an example of a motion in positive $y$-direction, where both motors lag the same amount in time.

In the diagram $\varphi_{1 \text { des }}$ and $\varphi_{2 \text { des }}$ are the desired motor angles at that point in time,

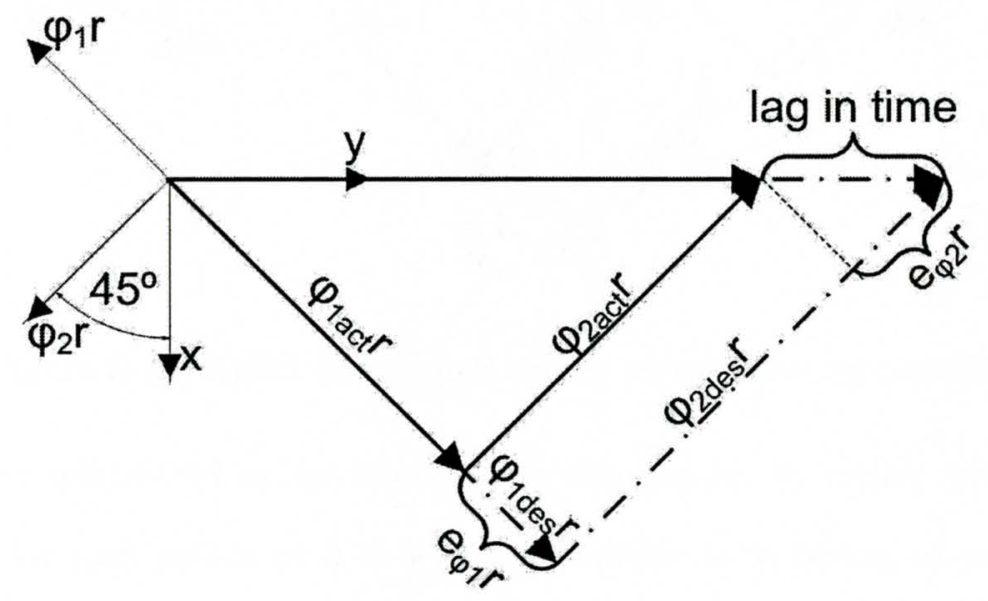

Figure 5.14. Effect of equal motor errors onto $x y$-position

while $\varphi_{1 a c t}$ and $\varphi_{2 a c t}$ are the actual (experimental or simulated) motor angles at that time instant. In Figure 5.14 the motor errors $e_{\varphi_{1}} r$ and $e_{\varphi_{2}} r$ have the same length that means the motor angles lag the desired motor angles by the same amount. This, as it can be seen in the figure, results in an overall error $\mathbf{e}$ along the negative $y$-axis. This again for a positive $y$-motion would only result in a lag in time, but not in an cross-axis error.

If however one of the errors is larger then the other, then the overall error vector would no longer be only in negative $y$-direction, but also would have an $x$ component. This $x$-component is what is causing the offset in $x$-direction while moving along the leg in $y$-direction. Figure 5.15 shows this effect for motor 2 error $\left(e_{\varphi_{2}} r\right)$ being larger than the motor 1 error $\left(e_{\varphi_{1}} r\right)$.

Those observations on the cause of the offset in each leg suggests that the 


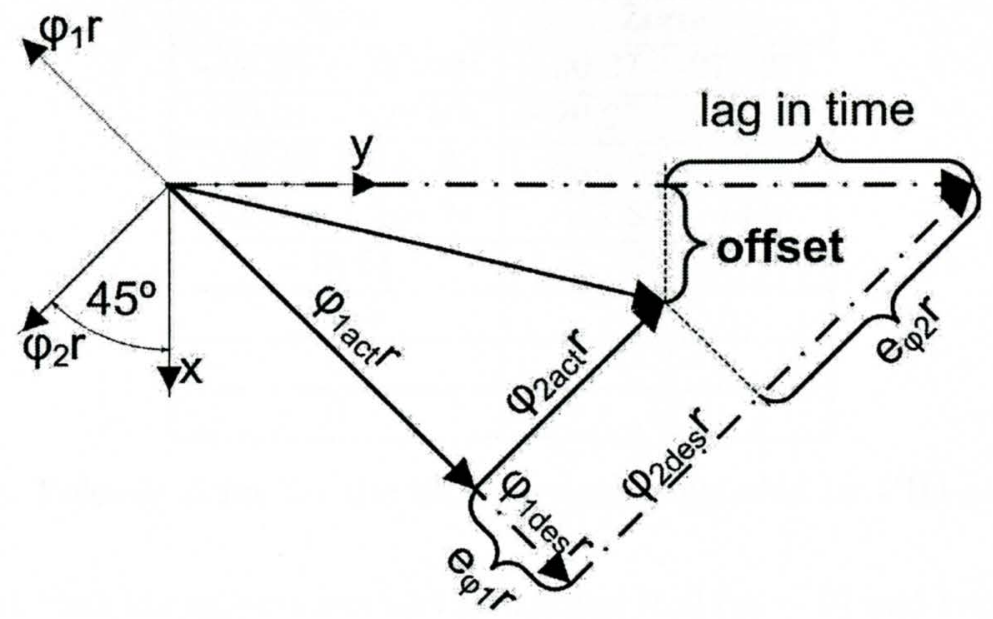

Figure 5.15. Effect of unequal motor errors onto $x y$-position

offset can be minimized or optimally even eliminated, by either eliminating the lag in time for each motor or if that is not possible then trying to generate equal absolute errors in both motors to minimizing the deviation from the shape.

\subsubsection{PID-controller Final Value Theorem}

Lets first examine if theoretically zero error in the motor angles is possible. For this examination we look only at one input output pair, such as for example motor 1 voltage input and motor 1 angle output. For this purpose the state space description of the linear $8^{\text {th }}$ order system in chapter 4 , needs to be slightly altered. First the input matrix $\mathbf{B}$ is changed into an input vector $\mathbf{b}$ by using only the first column of $\mathbf{B}$, which describes the input to motor one. Also the output matrix $\mathbf{C}$ needs to be changed into an output vector $\mathbf{c}$ that looks like the following.

$$
\mathbf{c}=\left[\begin{array}{llllllll}
0 & 0 & 0 & 0 & 1 & 0 & 0 & 0
\end{array}\right]
$$

Those changes give us a description for the SISO system which has the motor voltage 1 as an input and the motor angle 1 as an output, which is the system controlled by the PID-controller 1. Making those changes Matlab gives the following poles and zeros for that system. 


\begin{tabular}{|c|c|}
\hline Poles & Zeros \\
\hline$-20.01+329.03 i$ & $-20.27+279.97 i$ \\
\hline$-20.01-329.03 i$ & $-20.27-279.97 i$ \\
\hline$-21.98+249.3 i$ & $-17.82+144 i$ \\
\hline$-21.98-249.3 i$ & $-17.82-144 i$ \\
\hline-15.61 & -23.15 \\
\hline-43.9 & 0 \\
\hline 0 & $/$ \\
\hline 0 & $/$ \\
\hline
\end{tabular}

Table 5.2. Poles \& Zeros for the SISO system controlled by PID-controller 1

The fact that the system has one zero equal to $0\left(z_{6}=0\right)$ and two poles equal to $0\left(s_{7}=s_{8}=0\right)$ suggests that it has a transfer function $G(s)$ of the form shown in equation 5.14 .

$$
G(s)=\frac{s(\text { numeratorpolynomial }}{\left.s_{\text {rest }}(s)\right)}
$$

Where (numeratorpolynomial $l_{\text {rest }}(s)$ is the numerator polynomial after factorizing $s$ out and denominatorpolynomial ${ }_{\text {rest }}(s)$ is the denominator polynomial after factorizing $s^{2}$ out. That an $s$ in the numerator and an $s^{2}$ in the denominator can be factored out can be seen by looking at the zeros and poles of the system respectively.

The PID-controller transfer function $C(s)$ is

$$
C(s)=\frac{K_{D} s^{2}+K_{P} s+K_{I}}{s} .
$$

The closed loop error $e(s)$ can be derived from the block diagram in Figure 5.1 as:

$$
e(s)=\frac{r(s)}{1+C(s) G(s)}
$$

Where $r(s)$ is is the desired input signal, and $C(s)$ and $G(s)$ the controller and plant transfer functions respectively.

The input signal $r(s)$ can be characterized as a ramp during the steady state, the travel with constant velocity. Which is also the state in which the offset occurs. 
Therefore the input $r(s)$ has in the s-domain the form

$$
r(s)=\frac{m}{s^{2}} \quad[11]
$$

where $m$ is the slope of the ramp. To verify if the PID-controller can theoretically eliminate the motor angle error entirely we want to look at the steady state error $e_{s s}$. The steady state error is defined as the remaining error in steady state for $t \rightarrow \infty$. In order to find that error at $t \rightarrow \infty$ the so called Final Value Theorem[11] is used.

$$
\lim _{t \rightarrow \infty} e(t)=\lim _{s \rightarrow 0}[s e(s)]
$$

Where $e(t)$ is the error function in the time domain.

Therefore the steady state error in this case is given by the following relation.

$$
e_{s s}=\lim _{s \rightarrow 0}\left[s \frac{r(s)}{1+C(s) G(s)}\right]
$$

Substituting equations $5.14,5.15$ and 5.17 into equation 5.19 gives

$$
e_{s s}=\lim _{s \rightarrow 0}\left[s \frac{m}{s^{2}} \frac{1}{1+\frac{K_{D} s^{2}+K_{P} s+K_{I}}{s} \frac{s\left(\text { numeratorpolynomial }_{\text {rest }}(s)\right)}{s^{2}\left(\text { denominatorpolynomial }_{\text {rest }}(s)\right)}}\right] .
$$

Which can be simplified to

$$
e_{s s}=\lim _{s \rightarrow 0}\left[\frac{m s}{s^{2}+\left(K_{D} s^{2}+K_{P} s+K_{I}\right) \frac{\text { numeratorpolynomial } \text { dest }_{\text {denominatorpolynomial }(s)}(s)}{\text { dest }}}\right] .
$$

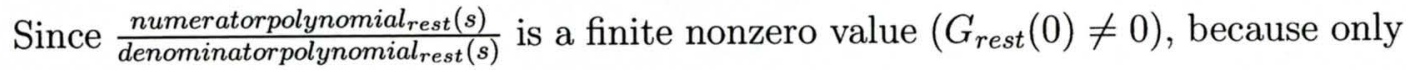
nonzero poles and zeros are left in those polynomials, the expression further simplifies to

$$
e_{s s}=\frac{0}{0+K_{I} G_{r e s t}(0)}=0
$$

This shows that theoretically a PID-controller controlling such a system can make the system follow a ramp without steady state error. Which leads to the question why we do see an error tracking the desired motor angle input (see Figure 5.12). 
The answer is that the steady state error is calculated for $t \rightarrow \infty$, but in the short time in which the motor actually is supposed to track a ramp before the intended deceleration starts to change direction, the response does not get into steady state mode.

\subsubsection{Controller Gains $K_{P}=150, K_{I}=1500$ and $K_{D}=4$}

An increasing of the $K_{I}$-gain could theoretically help reach a steady state error of zero quicker. To verify the effect of an increase of $K_{I}$ onto the system the $K_{I}$ values in both controllers are being increased to 1500 and the simulation is being repeated (see Figure 5.17.

Looking at the plot comparing the desired and the actual motor angles (Fig-

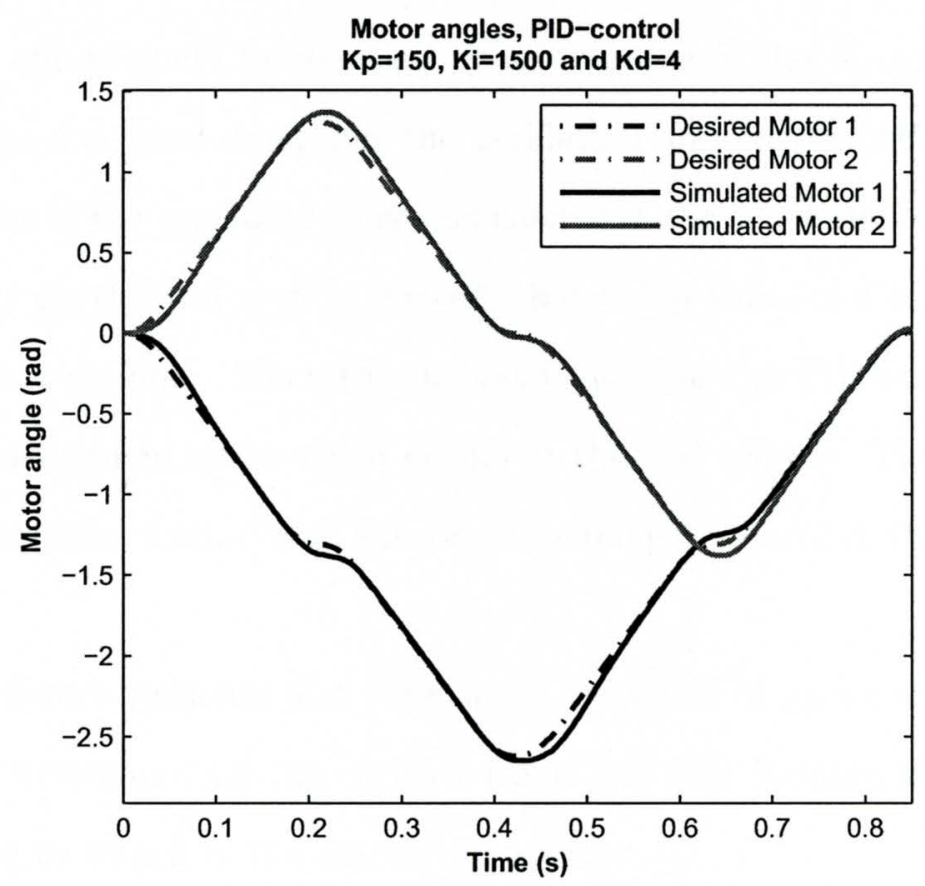

Figure 5.16.

ure 5.16) shows that indeed now the actual motor angle response gets pulled quicker towards an zero error during the ramp input sections and in some occasion seems even to turn out to be zero. However what also can be seen is, that it now per- 
forms rather poorly at those sections where the motors get decelerated, where it now overshoots. This is a direct consequence from increasing the $K_{I}$-gains. The integral part acts on the error history, but does not react to a change in error quickly enough. The change in the sign of the error when overshooting the desired trajectory, does decrease the error integral, however this is only done rather slowly since the error integral is increasing during the whole time while it was lagging the input. This delay in reacting to the change in the input signal causes the output to overshoot. And furthermore it is expected that if the $K_{I}$-gains are increased further, that already during the ramp section an overshoot occurs, since the integral output does not decrease until the error sign changes. This would cause an oscillation around the desired input and further decrease the quality of the result. Both those effects could be lessened by an increase of the $K_{D}$-gains. Since that would on the one hand damp out the oscillation and on the other hand increase the quickness of the controller to act on change in the input signal. Unfortunately though tests on the real system showed, that a $K_{D}$ value of 4 is already close to the maximum possible. Since the derivative part of the PID-controller tends to amplify measurement noise which occurs in the real system. This would quickly drive the controller output into saturation making a control of the system impossible.

The end-effector response also reveals the weakness of an increasing of the $K_{I^{-}}$ gains. The Overshoot on the corners are so big that it takes almost the whole following leg to return to the desired $x y$-position.

Table 5.3 shows the deviations from the desired shape for this PID-setup. It shows the major increase in overshoot at the corners, while an improvement in offset is actually reached. However those offset values are rather theoretical in nature, since it takes the system a majority of the length of each leg to actually return from the 


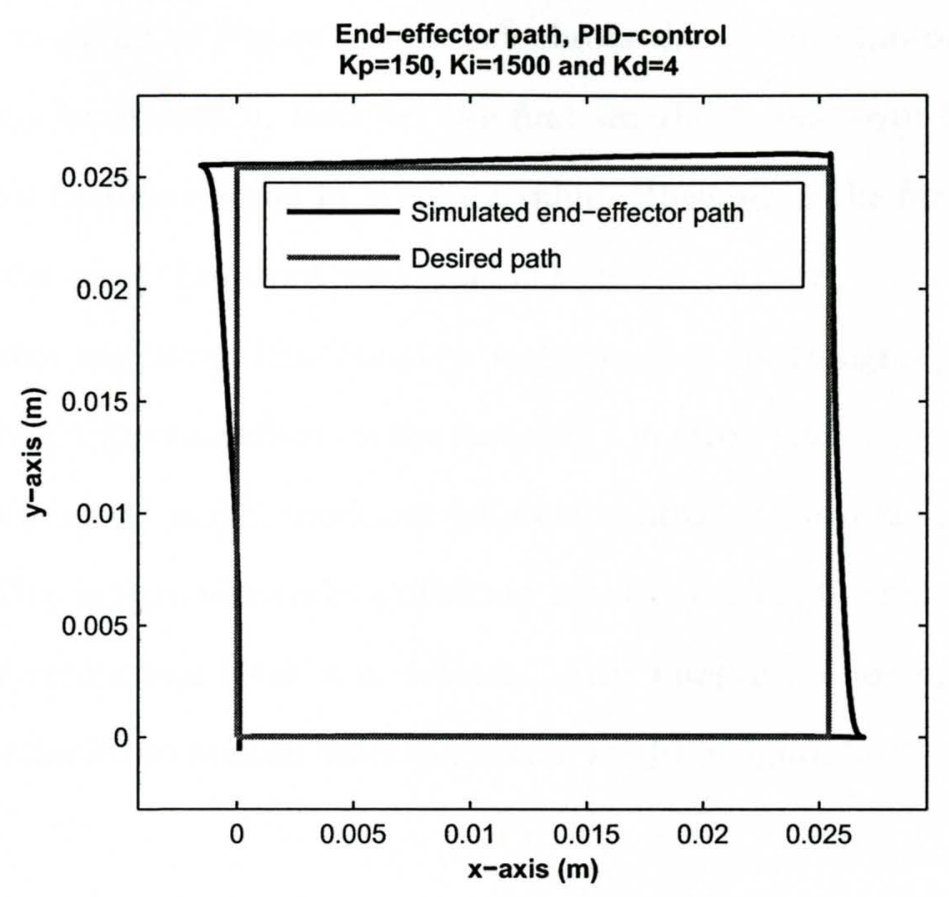

Figure 5.17.

huge overshoot to a steady state offset that is why for most of the lag the offset is bigger than the steady state offset shown in the table.

\begin{tabular}{|c|c|c|c|c|}
\hline & $1^{\text {st }}$ leg & $2^{\text {nd }}$ leg & $3^{\text {rd }}$ leg & $4^{\text {th }}$ leg \\
\hline $\begin{array}{c}\text { Overshoot } \\
\text { at the end of the leg } \\
\text { in } m\end{array}$ & $1.52 \times 10^{-3}$ & $6.32 \times 10^{-4}$ & $1.55 \times 10^{-3}$ & $6.2 \times 10^{-4}$ \\
\hline $\begin{array}{c}\text { Offset } \\
\begin{array}{c}|\max | \\
\text { in } m\end{array}\end{array}$ & $3.9 \times 10^{-5}$ & $1.2 \times 10^{-4}$ & $1.39 \times 10^{-4}$ & $1.06 \times 10^{-4}$ \\
\hline
\end{tabular}

Table 5.3. Path deviations PID-control; $K_{p}=150, K_{I}=1500$ and $K_{D}=4$

\subsubsection{Influence of Integral Action on Motor Error Inequality}

Since a total elimination of the motor angle tracking error is not possible, we look next to see if it is possible to get equal tracking errors for both motors so that the resulting error is only a lag in time, but does not effect the shape. In order to eliminate the error inequality we first examine what is causing this 
inequality. Looking at Figure 5.13 which shows the error in motor angle for each motor brings to attention, that for the first leg the errors seem to be equal but opposite and therefore equal in absolute value. However in the further progress of the graph the errors built out very different curves and are generally not equal in absolute value anymore. This behavior suggests that the integral part of the PIDcontroller might have an effect on the inequality in error, since that part acts on the history of the error, and it produces different controller outputs for different error histories. This in turn generates a different input to each of the motors, which leads to different errors and thus to a different error integral. Looking at the desired motor trajectories compared with the actual angles (Figure 5.12) strengthens this suspicion.

At the beginning of the run both motors start with an error integral of zero. Since both motors lag behind the desired values, they both built up their error integrals rather equally. However now when starting the second lag the direction of motor 2 changes while motor 1 keeps turning in the same direction. That means that the error integral of motor 1 further windup while the error of motor 2 changes sign causing the error integral to decrease. This leaves the motors with totally different integral controller output which effects the overall controller output. Figure 5.18

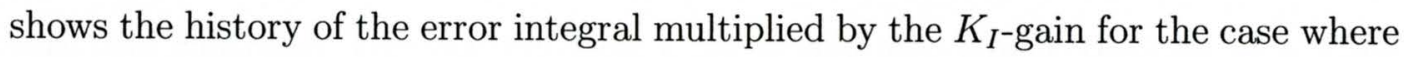
$K_{I}=100$.

\subsection{PD-controller performance in simulation}

Now that we identified a source for the inequality of the motor errors a strategy has to be found to avoid the inequality and therefore improve the tracking of the desired path. The influence of the different error integrals would be eliminated if the $K_{I}$-gains would be set to zero and therefore instead of an PID-controller a PD-controller is being used. 


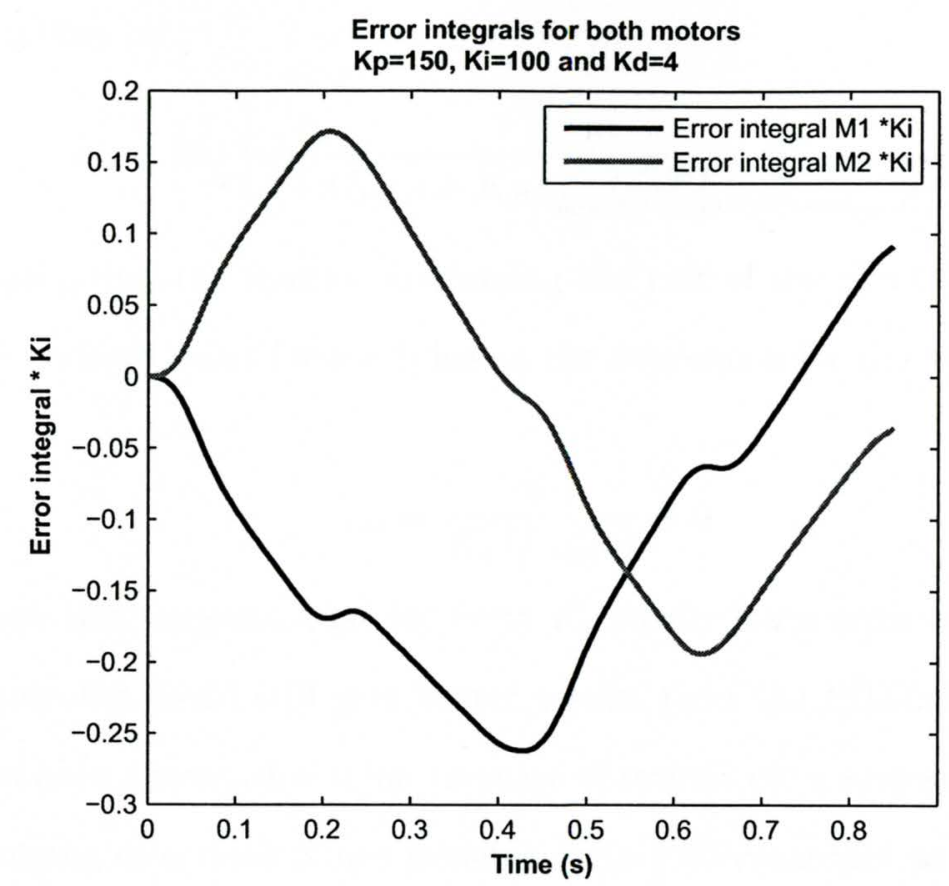

Figure 5.18.

\subsubsection{PD-controller final value theorem}

In order to see the ability of such an controller to track a ramp, which is still the major component of the desired trajectory the final value theorem is being stressed one more time. The expression for the error stays the same as for the PID-controller (equation 5.16) as well as the plant transfer function $G(s)$ (equation 5.14). However now the controller transfer function $C(s)$ is not any longer the one of a PIDcontroller but the one of an PD-controller shown in equation 5.23.

$$
C(s)=K_{D} s+K_{P}
$$

With those changes the steady state error derived from the final value theorem (equation 5.18) is.

$$
e_{s s}=\lim _{s \rightarrow 0}\left[s \frac{m}{s^{2}} \frac{1}{1+\left(K_{D} s+K_{P}\right) \frac{s\left(\text { numeratorpolynomial }_{\text {rest }}(s)\right)}{s^{2}\left(\text { denominatorpolynomial }_{\text {rest }}(s)\right)}}\right]
$$


Which simplifies to

$$
e_{s s}=\lim _{s \rightarrow 0}\left[\frac{m}{s+\left(K_{D} s+K_{P}\right) \frac{\text { numeratorpolynomial } \text { dest }_{\text {denominatorpolynomial }}(s)}{\text { dest }}(s)}\right] .
$$

Again arguing that the fraction containing the rest of the plant transfer function is an finite nonzero value for $s=0$ leaves the expression for the steady state error as

$$
e_{s s}=\frac{m}{K_{P} G_{r e s t}(0)} \neq 0
$$

Even though this suggests, that for finite $K_{p}$ steady state error will never vanish, the PD-controller could still give better results then the PID-controller. This is since it has been shown, due to an increase of overshoot, a zero steady state error before changing directions is not possible for a PID-controller as well. Therefore since the PD-controller reduces the inequality in motor error, which causes the offset and since the value of the steady state error can be minimized by increasing $K_{P}$, the PD-controller might perform better than the PID-controller.

\subsubsection{Controller Gains $K_{P}=150$ and $K_{D}=4$}

Again simulation is being carried out to quantify the improvement. Figure 5.19 shows the end-effector path for that case. From this plot already an improvement in offset can be made out especially in the second leg.

Figure 5.20 shows the error in shape. It shows also the improvement in offset. If we look at the error in motor angle for this case (Figure 5.21) we can see that the motor errors for both motors are more equal then for the PID-case. Which proves the point, that the integral part of the controller was a big source for the inequality. Table 5.4 shows the summarized errors of the end-effector position. 


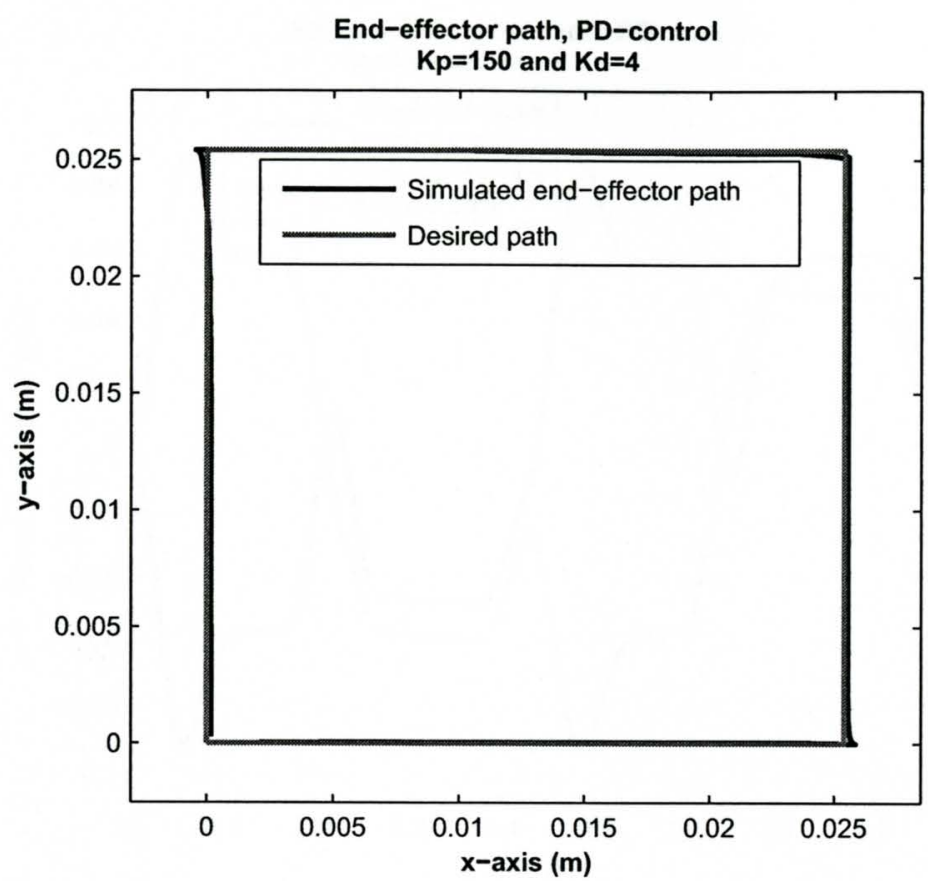

Figure 5.19.

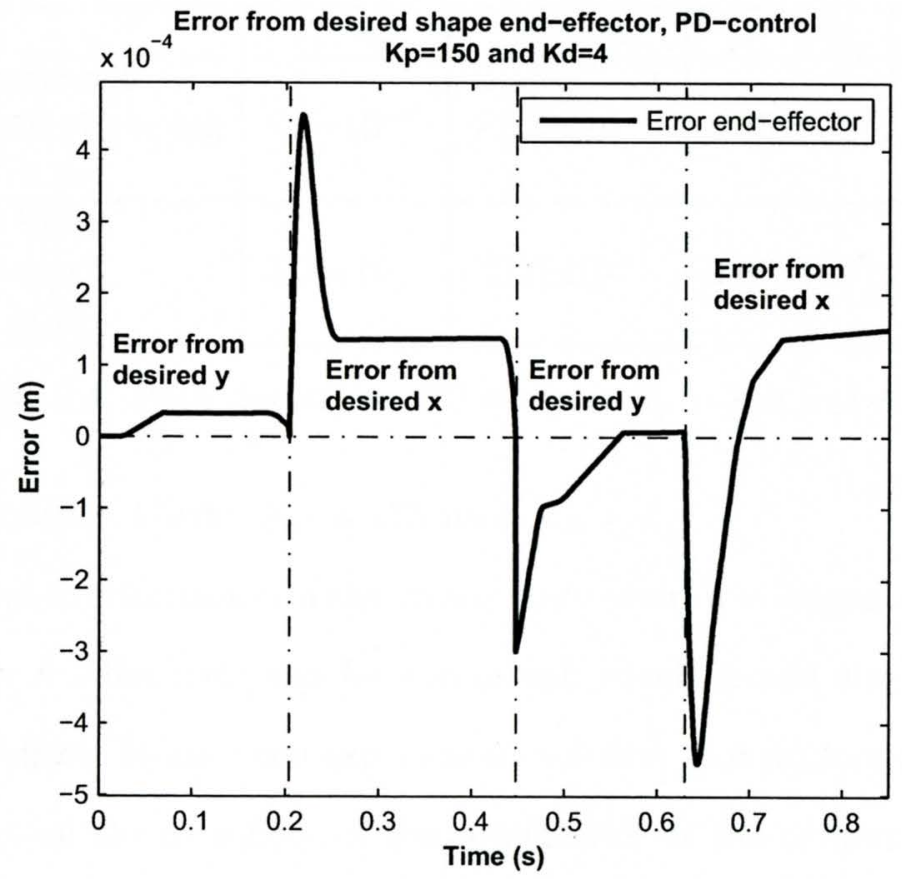

Figure 5.20. 


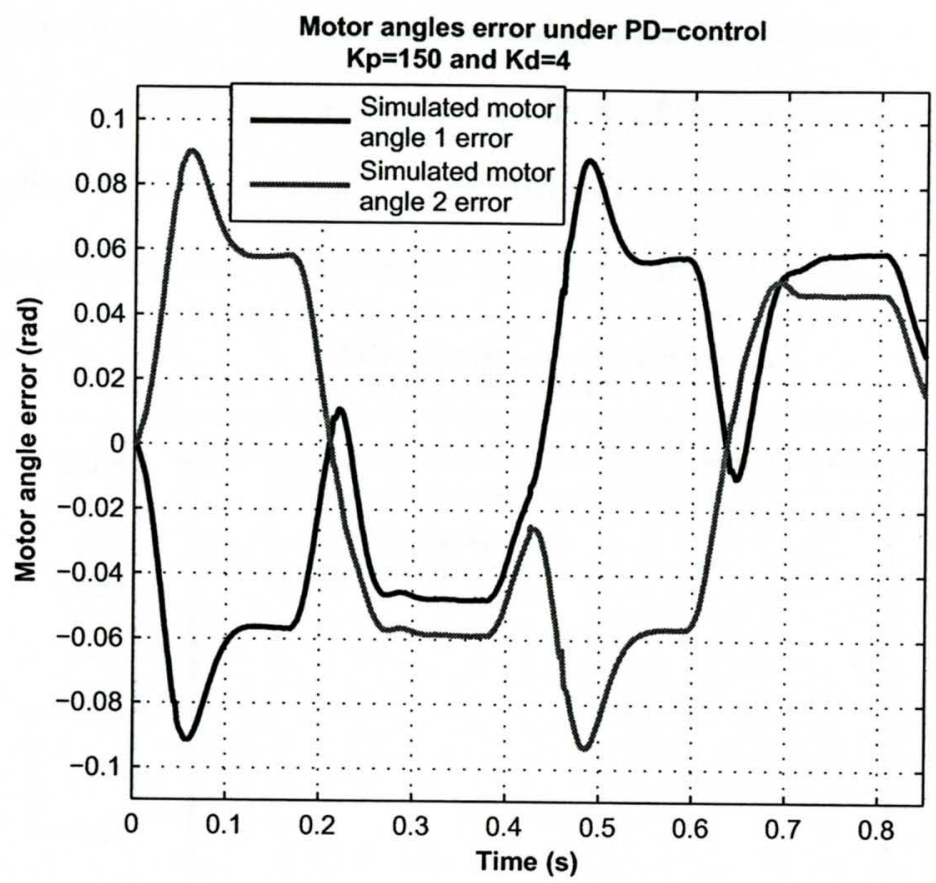

Figure 5.21.

\begin{tabular}{|c|c|c|c|c|}
\hline & $1^{\text {st }}$ leg & $2^{\text {nd }}$ leg & $3^{\text {rd }}$ leg & $4^{\text {th }}$ leg \\
\hline $\begin{array}{c}\text { Overshoot } \\
\text { at the end of the leg } \\
\text { in } m\end{array}$ & $4.5 \times 10^{-4}$ & $\begin{array}{c}\text { Undershoot } \\
-2.99 \times 10^{-4}\end{array}$ & $4.55 \times 10^{-4}$ & $/$ \\
\hline $\begin{array}{c}\text { Offset } \\
|\max | \\
\text { in } m\end{array}$ & $3.24 \times 10^{-5}$ & $1.37 \times 10^{-4}$ & $0.67 \times 10^{-5}$ & $1.46 \times 10^{-4}$ \\
\hline
\end{tabular}

Table 5.4. Path deviations PD-control; $K_{p}=150$ and $K_{D}=4$

\subsubsection{Controller Gains $K_{P}=175$ and $K_{D}=4$}

Looking at the derivation of the steady state error, it is suggested, that through an increase of $K_{P}$ the error can be minimized, which should also have a positive effect on the offset. It has been experienced however that an increase in $K_{P}$ has a negative effect on the overshoot of the end-effector at the corners. Increasing the $K_{D}$-gain in turn has a positive effect on the overshoot. However as mentioned earlier the $K_{D^{-}}$gain is limited in the real system due to its characteristic of amplifying 
the measurement noise. Simulations revealed that an increase of the proportional gain to $K_{P}=175$ gives better results in offset while keeping the overshoot at the level of the first PID case. Figures 5.22 through 5.24 show the result for that case. The shape of the motor error curves look very similar to the previous case, but it

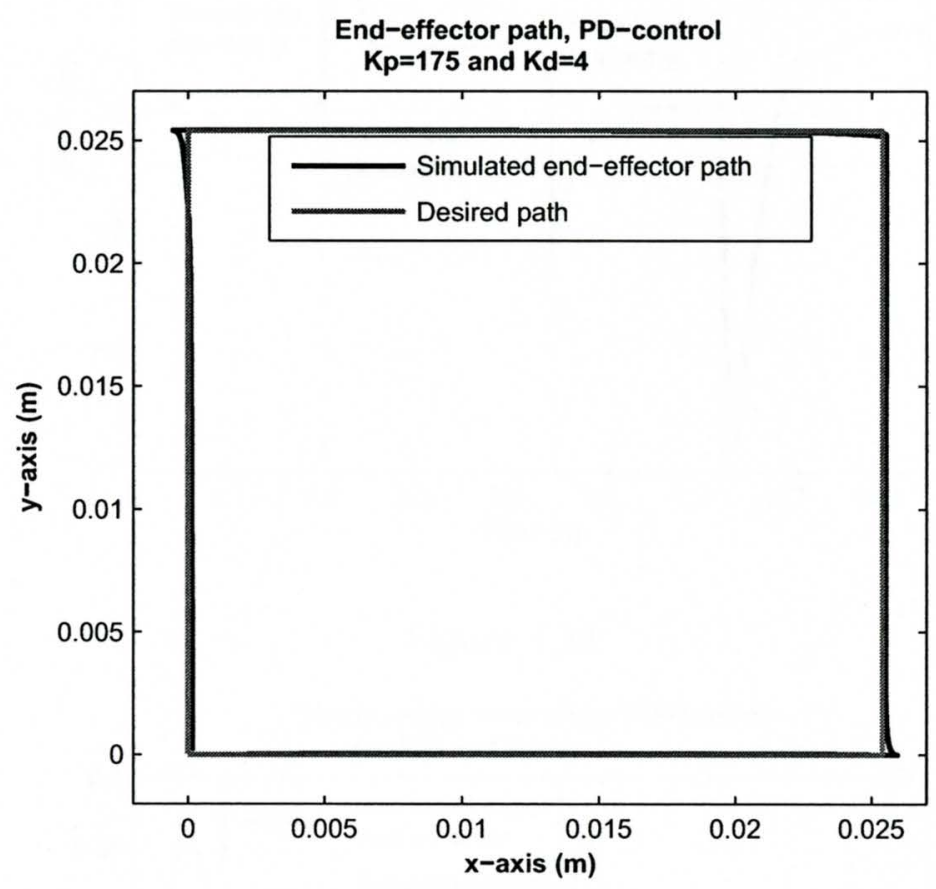

Figure 5.22.

can be seen that the total amount of error is less then in the previous case, which is the reason for the improvement in offset.

Table 5.5 shows that the new gains could improve the offset while keeping the

\begin{tabular}{|c|c|c|c|c|}
\hline & $1^{\text {st }}$ leg & $2^{\text {nd }}$ leg & $3^{\text {rd }}$ leg & $4^{\text {th }}$ leg \\
\hline $\begin{array}{c}\text { Overshoot } \\
\text { at the end of the leg } \\
\text { in } m\end{array}$ & $5.5 \times 10^{-4}$ & $\begin{array}{c}\text { Undershoot } \\
-2.15 \times 10^{-4}\end{array}$ & $5.54 \times 10^{-4}$ & $/$ \\
\hline $\begin{array}{c}\text { Offset } \\
|\max | \\
\text { in } m\end{array}$ & $3.37 \times 10^{-5}$ & $1.12 \times 10^{-4}$ & $1.05 \times 10^{-5}$ & $1.43 \times 10^{-4}$ \\
\hline
\end{tabular}

Table 5.5. Path deviations PD-control; $K_{P}=175$ and $K_{D}=4$ 


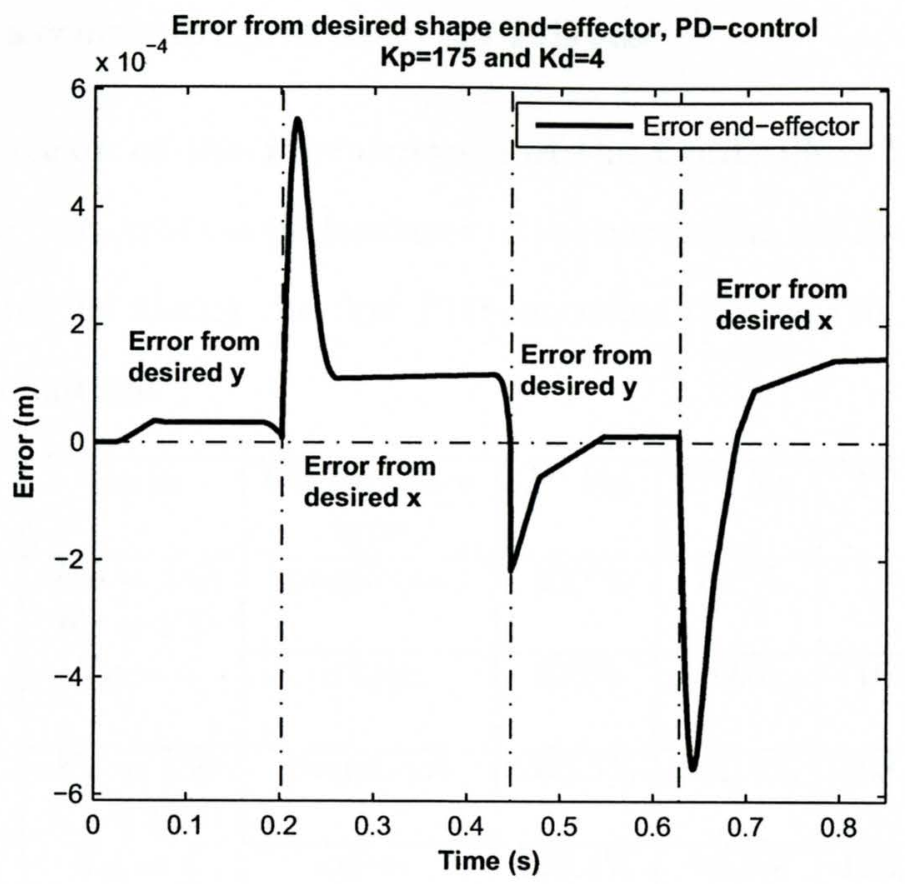

Figure 5.23.

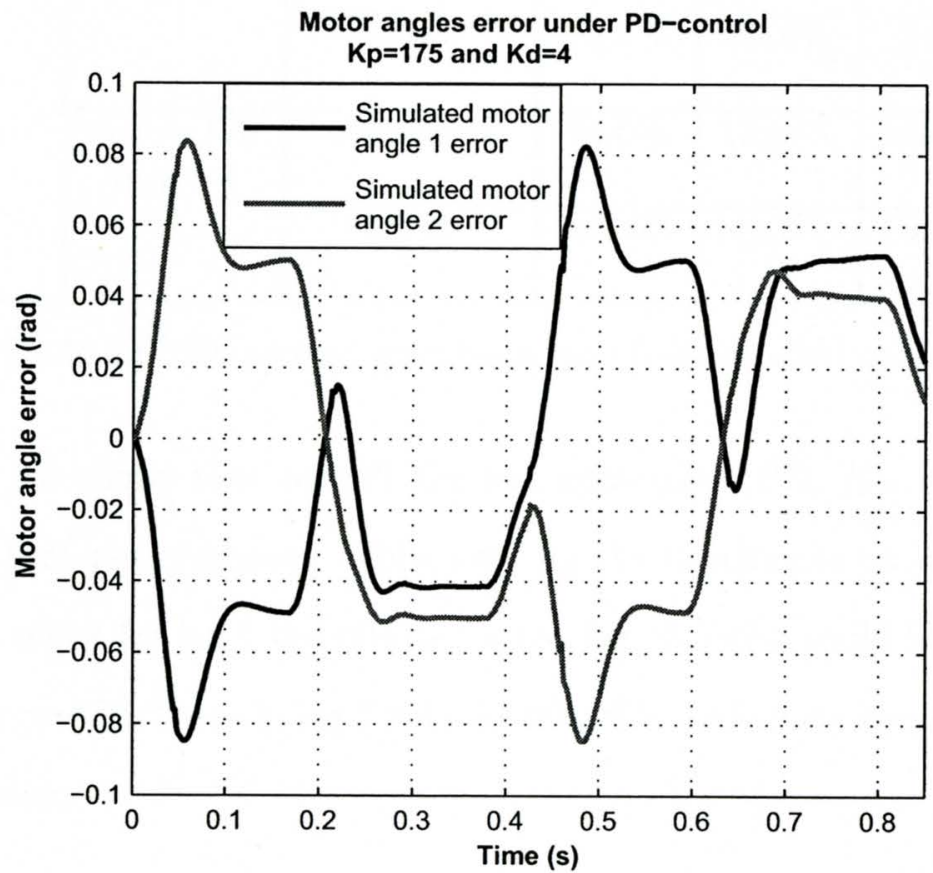

Figure 5.24. 
overshoot at a comparable level as for the PID case.

\subsection{Comparison of the Performance of the Controllers in Simulation}

Table 5.6 compares the performance of the controllers studied in this chapter with each other by taking the first PID-controller $\left(K_{P}=150, K_{I}=100\right.$ and $\left.K_{D}=4\right)$ as a baseline.

\begin{tabular}{|c|c|c|c|c|c|c|}
\hline $\begin{array}{c}\text { Controller } \\
\text { type }\end{array}$ & Gains & $\begin{array}{c}\text { Performance } \\
\text { type }\end{array}$ & $1^{\text {st }} \operatorname{leg}$ & $2^{\text {nd }} \operatorname{leg}$ & $3^{\text {rd }} \operatorname{leg}$ & $4^{\text {th }} \operatorname{leg}$ \\
\hline \multirow[t]{4}{*}{ PID } & \multirow{2}{*}{$\begin{array}{c}K_{P}=150 \\
K_{I}=100 \\
K_{D}=4\end{array}$} & overshoot & $100 \%$ & $100 \%$ & $100 \%$ & 1 \\
\hline & & offset & $100 \%$ & $100 \%$ & $100 \%$ & $100 \%$ \\
\hline & \multirow{2}{*}{$\begin{array}{c}K_{P}=150 \\
K_{I}=1500 \\
K_{D}=4\end{array}$} & overshoot & $260.5 \%$ & $361.1 \%$ & $308.8 \%$ & T \\
\hline & & offset & $116.4 \%$ & $44.1 \%$ & $120.9 \%$ & $101 \%$ \\
\hline \multirow[t]{4}{*}{$\mathrm{PD}$} & \multirow{2}{*}{$\begin{array}{c}K_{P}=150 \\
K_{D}=4\end{array}$} & overshoot & $77.3 \%$ & $175.9 \%$ & $90.6 \%$ & I \\
\hline & & offset & $96.7 \%$ & $50.4 \%$ & $5.8 \%$ & $139.1 \%$ \\
\hline & \multirow{2}{*}{$\begin{array}{c}K_{P}=175 \\
K_{D}=4\end{array}$} & overshoot & $94.5 \%$ & $122.8 \%$ & $110.4 \%$ & $T$ \\
\hline & & offset & $100.6 \%$ & $41.2 \%$ & $9.1 \%$ & $136.2 \%$ \\
\hline
\end{tabular}

Table 5.6. Compared performances of the studied controllers

Table 5.6 shows that overall the last controller (PD, $\left.K_{P}=175, K_{D}=4\right)$ shows the best performance. While keeping the overshoots on the same level as well as the offset for leg 1 the offsets for the legs 2 and 3 could be improved. The biggest improvement was in leg 3 with $90.9 \%$ offset reduction compared to the first PID-controller.

\subsection{Experimental Performance of the Controllers on the Real System}

As a next step, we need to investigate this controller performance on the real system. In order to change the PID-controller into an PD-controller no further 
programming steps have to be taken. The code of the PID reset windup algorithm shown in Appendix A can be used with the only change, that now the $K_{I}$ gains are set to be zero.

First the PID controller $\left(K_{P}=150, K_{I}=100\right.$ and $\left.K_{D}=4\right)$ is being tested as a baseline to check if the PD solution can give similar improvement in the real system as could be observed in simulation. Figure 5.25 shows the result, the deviations are summarized in Table 5.7.

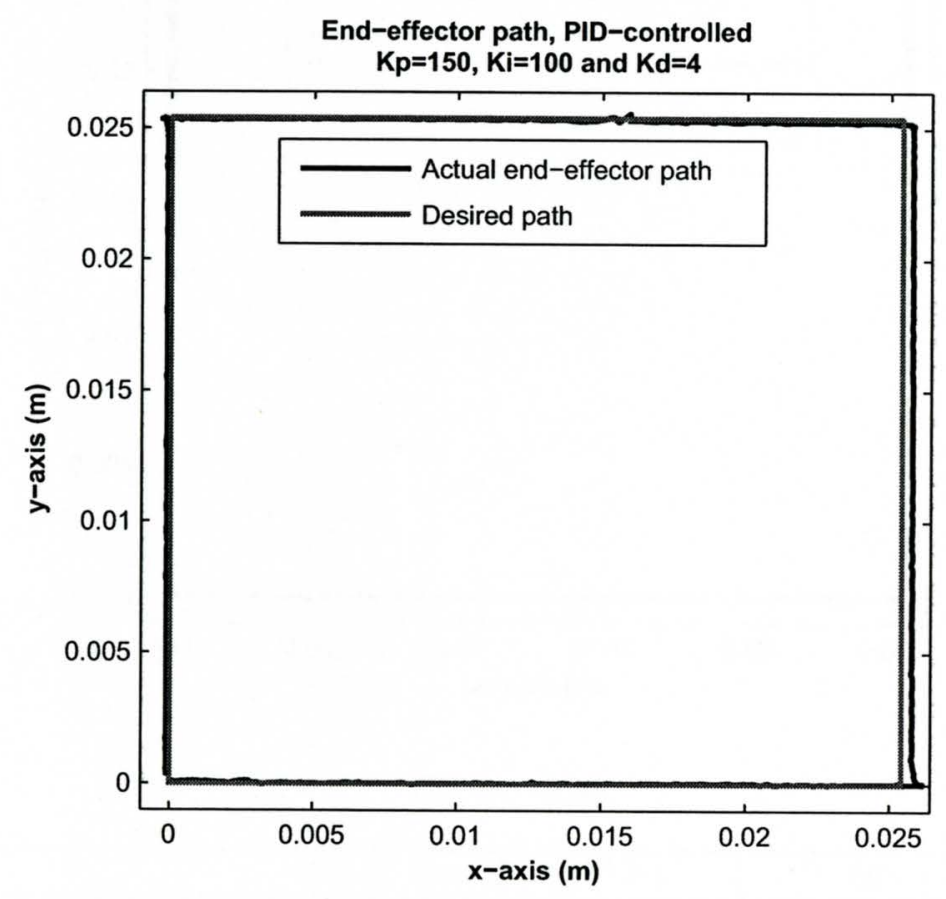

Figure 5.25.

Next the PD-controller with $K_{P}=175$ and $K_{D}=4$ is being tested. Figure 5.26 shows the end-effector path, while Table 5.8 summarizes the results.

Table 5.5 and 5.6 show that the offset in the legs 1,2 and 4 are improved, but an unexpected increase in offset for leg 3 is experienced. Looking at the plot of the error in motor angle (Figure 5.27) shows that Motor 1 seems to have a smaller 


\begin{tabular}{|c|c|c|c|c|}
\hline & $1^{\text {st }}$ leg & $2^{\text {nd }}$ leg & $3^{\text {rd }}$ leg & $4^{\text {th }}$ leg \\
\hline $\begin{array}{c}\text { Overshoot } \\
\text { at end of the leg } \\
\text { in } m\end{array}$ & $7.4 \times 10^{-4}$ & $\begin{array}{c}\text { Undershoot } \\
-2 \times 10^{-4}\end{array}$ & $3.2 \times 10^{-4}$ & $/$ \\
\hline $\begin{array}{c}\text { Offset } \\
\text { max } \mid \\
\text { in } m\end{array}$ & $1 \times 10^{-4}$ & $3.8 \times 10^{-4}$ & $1 \times 10^{-4}$ & $1.2 \times 10^{-4}$ \\
\hline
\end{tabular}

Table 5.7. Path deviations PID-control; $K_{P}=150, K_{I}=100$ and $K_{D}=4$

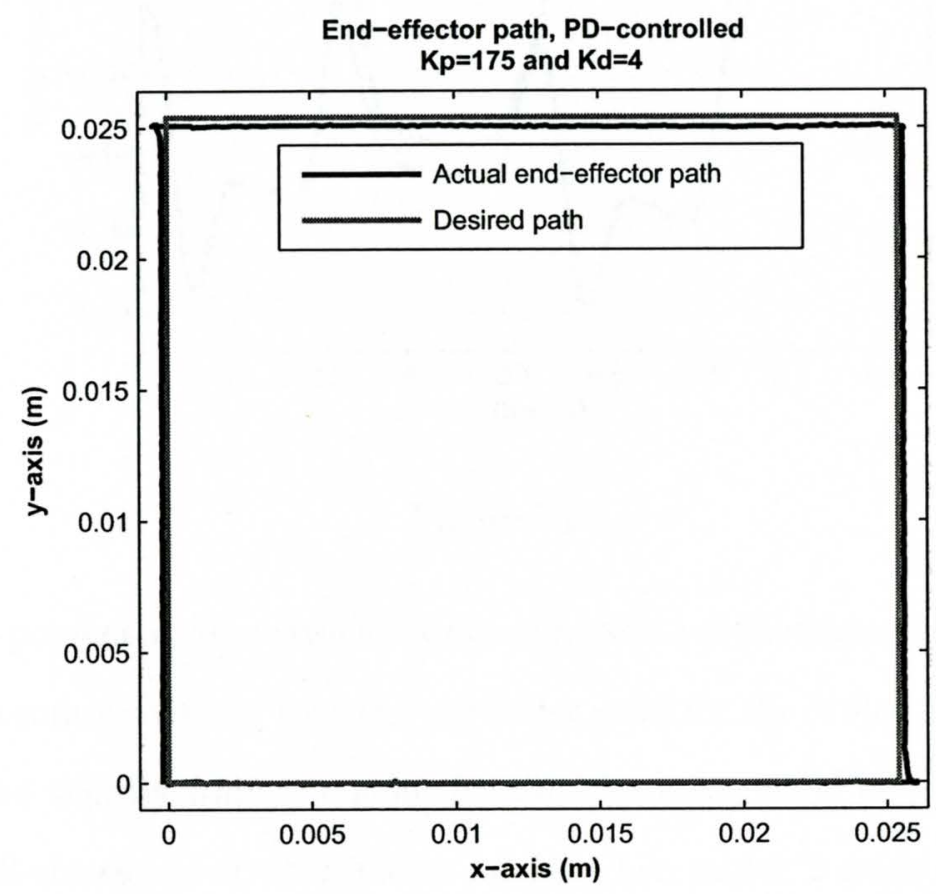

Figure 5.26.

\begin{tabular}{|c|c|c|c|c|}
\hline & $1^{\text {st }}$ leg & $2^{\text {nd }}$ leg & $3^{\text {rd }}$ leg & $4^{\text {th }}$ leg \\
\hline $\begin{array}{c}\text { Overshoot } \\
\text { at the end of the leg } \\
\text { in } m\end{array}$ & $6.2 \times 10^{-4}$ & $\begin{array}{c}\text { Undershoot } \\
-4 \times 10^{-4}\end{array}$ & $4.9 \times 10^{-4}$ & $/$ \\
\hline $\begin{array}{c}\text { Offset } \\
\text { max } \\
\text { in } m\end{array}$ & $7 \times 10^{-5}$ & $2 \times 10^{-4}$ & $3.4 \times 10^{-4}$ & $1.9 \times 10^{-4}$ \\
\hline
\end{tabular}

Table 5.8. Path deviations PD-control; $K_{P}=175$ and $K_{D}=4$

error over the whole run.

A reason for that could be, that Motor 1 is slightly stronger then Motor 2, which 


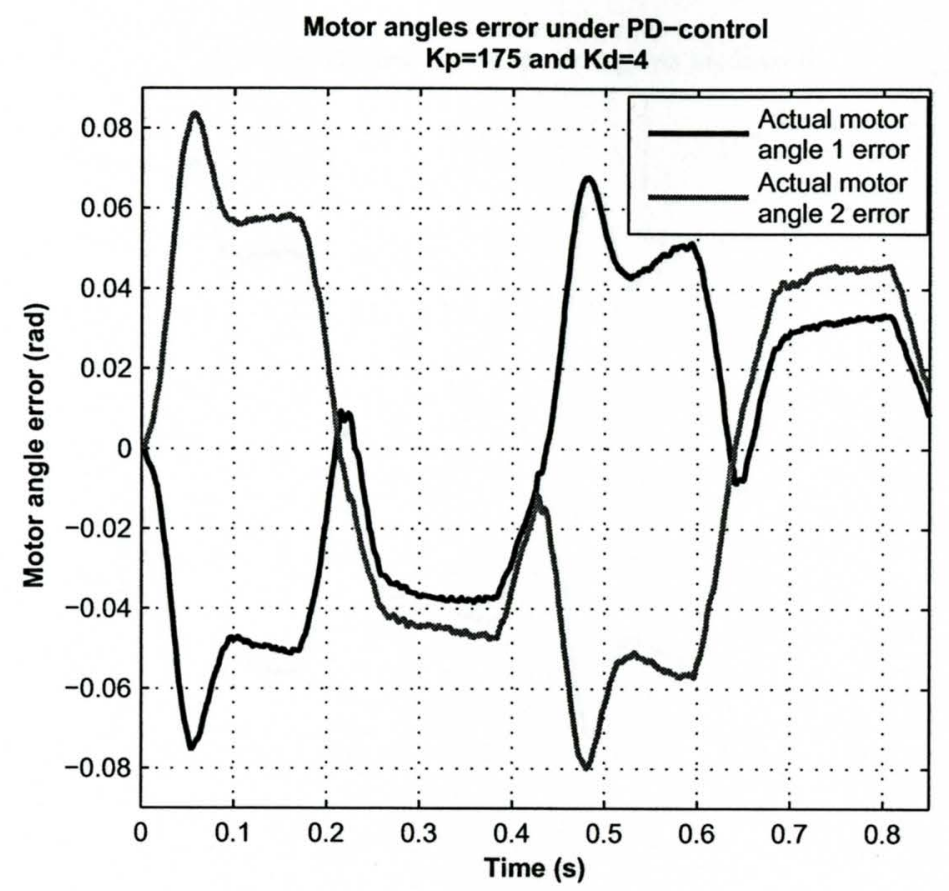

Figure 5.27.

can not be prevented, since two motors can never have the same exact performance. In order to compensate for that the controller gains for the Motor 2 control loop can be increased slightly while the gains for the Motor 1 control loop stay unchanged. Figure 5.28 shows the error in motor angles when motor 2 gains are increased by $10 \%$.

Compared to the case with equal controller gains for both motors, the error

\begin{tabular}{|c|c|c|c|c|}
\hline & $1^{\text {st }}$ leg & $2^{\text {nd }}$ leg & $3^{\text {rd }}$ leg & $4^{\text {th }}$ leg \\
\hline $\begin{array}{c}\text { Overshoot } \\
\text { at the end of the leg } \\
\text { in } m\end{array}$ & $7.4 \times 10^{-4}$ & $\begin{array}{c}\text { Undershoot } \\
-1.4 \times 10^{-4}\end{array}$ & $4.3 \times 10^{-4}$ & $/$ \\
\hline $\begin{array}{c}\text { Offset } \\
|\max | \\
\text { in } m\end{array}$ & $7 \times 10^{-5}$ & $2.3 \times 10^{-4}$ & $1.2 \times 10^{-4}$ & $8 \times 10^{-5}$ \\
\hline
\end{tabular}

Table 5.9. Path deviations PD-control increased gains Motor 2

plot now looks more equal. And consequently the offsets improved, as shown in 


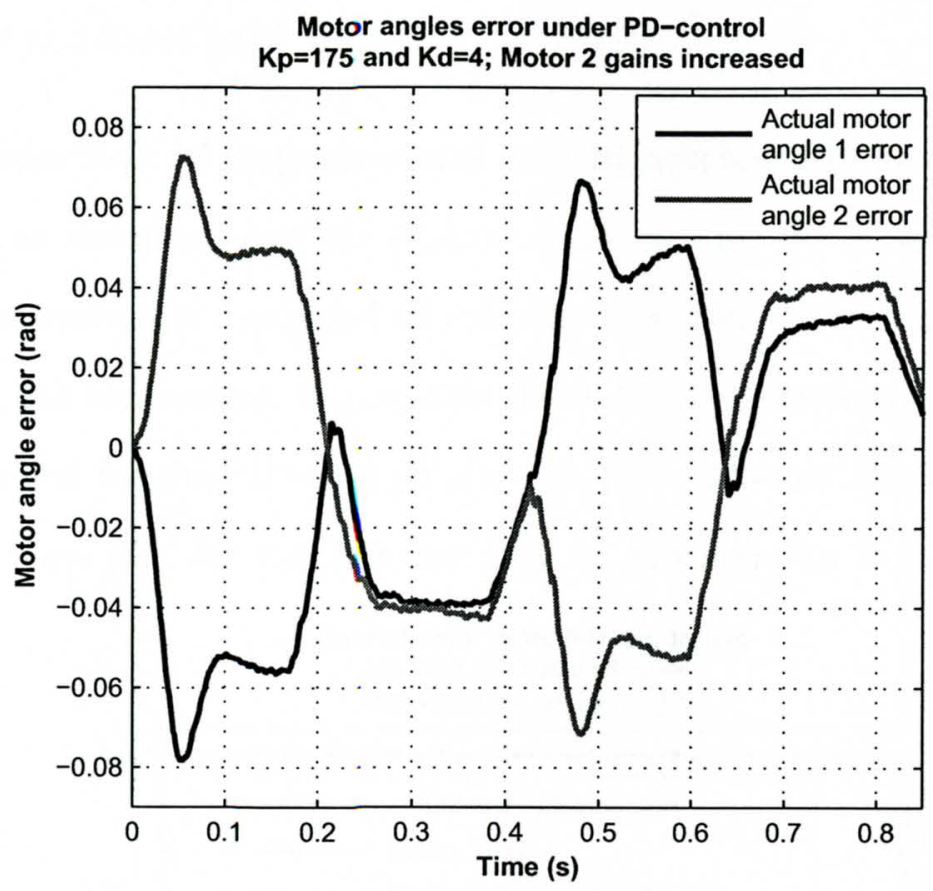

Figure 5.28.

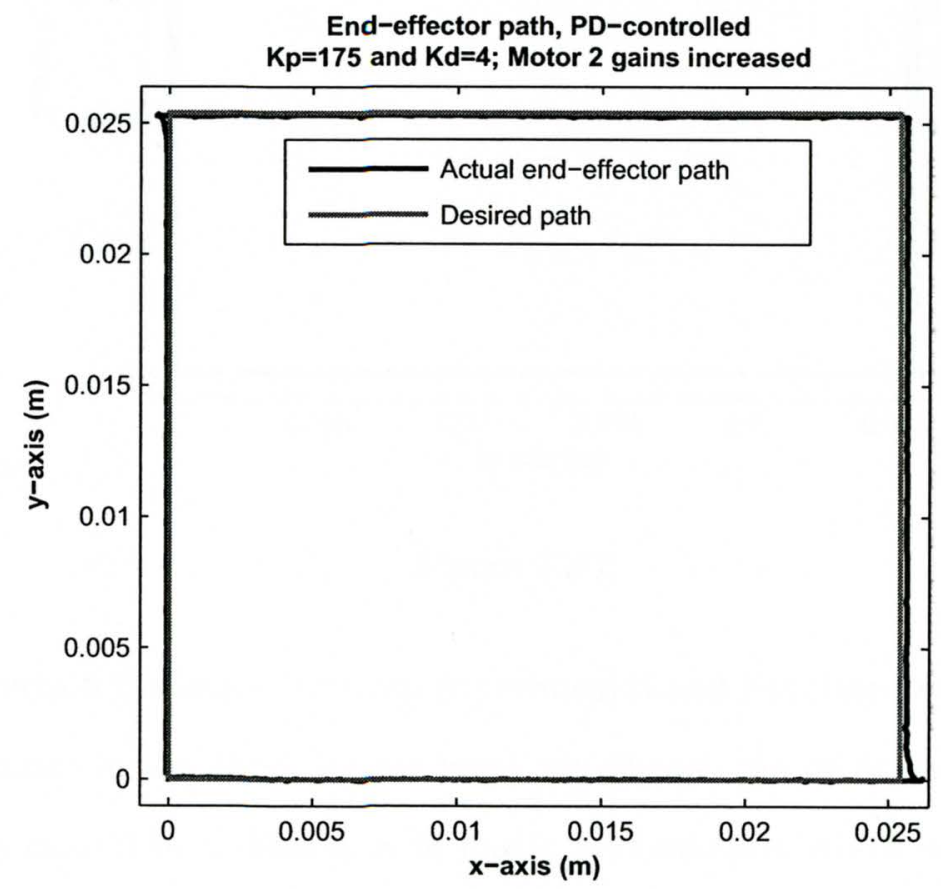

Figure 5.29. 
Figure 5.29 and Table 5.9.

\subsection{Comparison of Experimental and Simulated Performance}

Next the simulated and the experimental data for the closed loop control of the H-frame system is compared to evaluate how well the model can predict the response of the real system. Figure 5.30 shows the end-effector path for simulation and experiment for the PID-case $\left(K_{P}=150, K_{I}=100\right.$ and $\left.K_{D}=4\right)$, Figure 5.31 shows the same plot for the PD-case $\left(K_{P}=175\right.$ and $\left.K_{D}=4\right)$. Figure 5.31

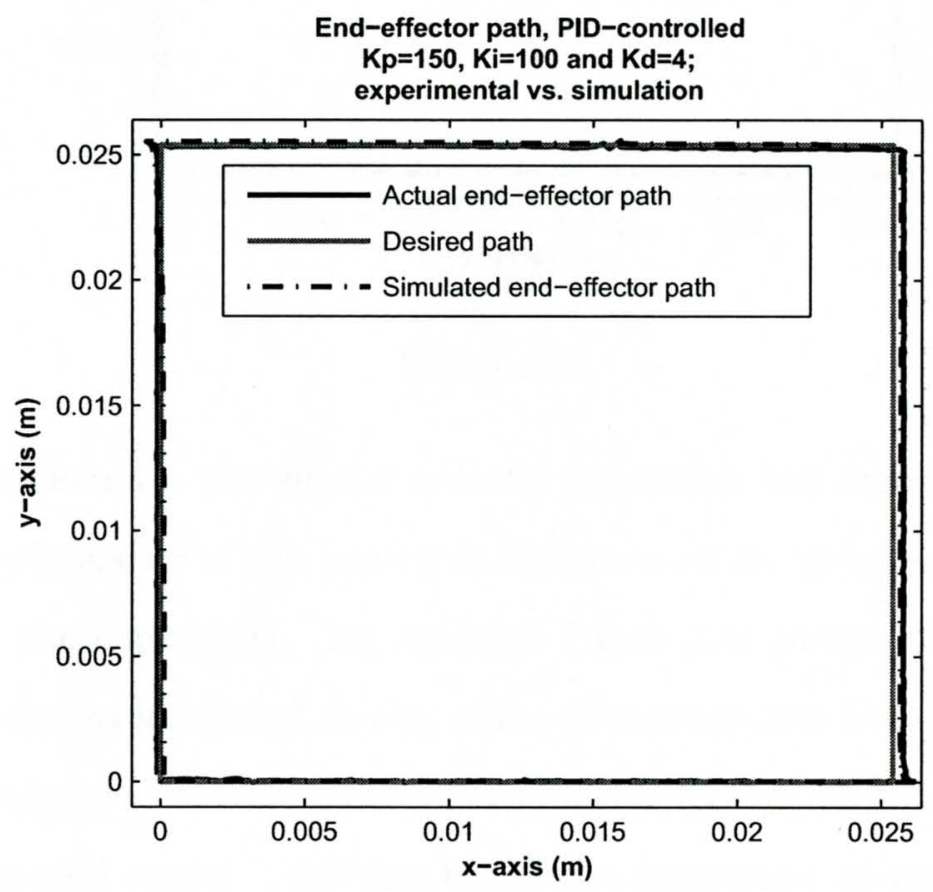

Figure 5.30.

reveals a certain deviation between experimental and simulated end-effector path. The deviations in the third leg are most significant. As mentioned before this is most likely caused by a difference in motor performance, which is not reflected in the dynamic model. Compensating this by increasing the controller gains for the weaker motor, motor 2 , should minimize the deviations of simulation and experimental path. 


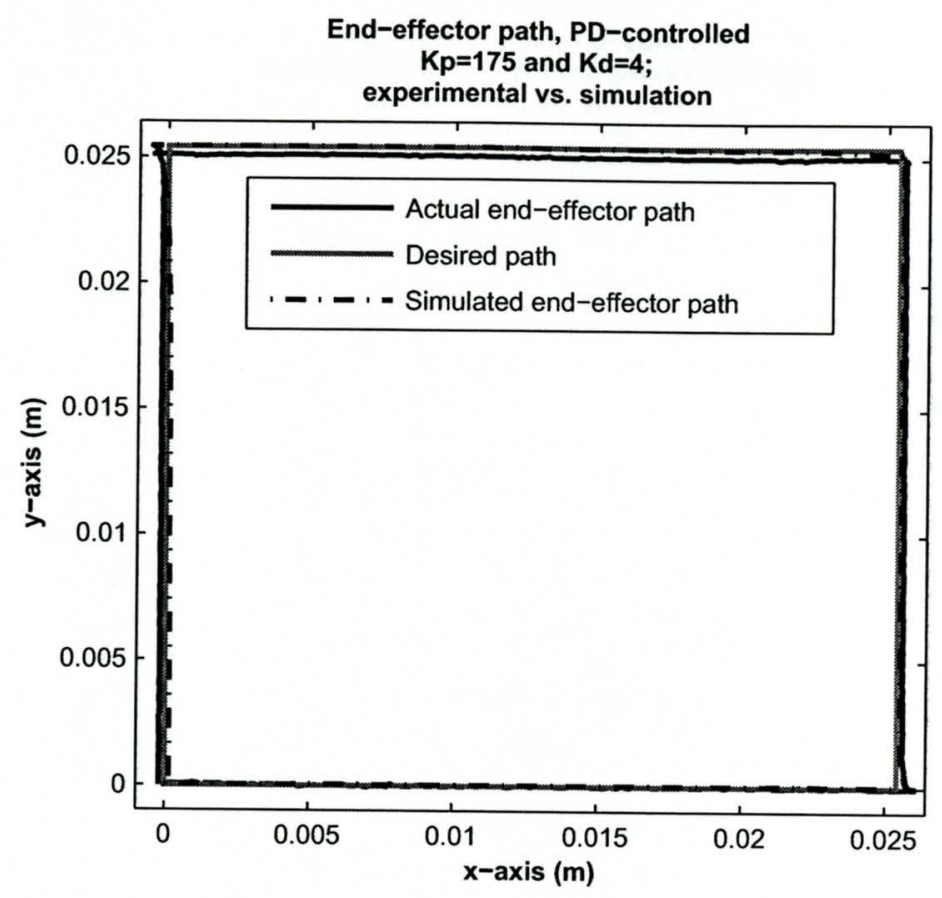

Figure 5.31.

Figure 5.32 shows the end-effector path for simulation and experiment where the different performance of the motors is compensated by increasing the gains for motor 2 in the experiment. As expected Figure 5.32 shows a better match of simulation and experimental results. This strengthens the assumption, that the different performance of the motors is one factor for the deviation of simulation and experimental results. Therefore for further comparison of simulation and experimental results the experimental motor 2 gains will stay increased, in order to compensate for the difference in motor performance.

Even though a certain deviation is still observable it can also be seen that the model reflects the dynamics of the real system generally well. It can be observed in Figure 5.32 that size and shape of the overshoots at the end of leg one and three are matched well by the simulation. Also the tendency of the real system to undershoot at the end of leg 2 is reflected by the simulation based on the nonlinear 


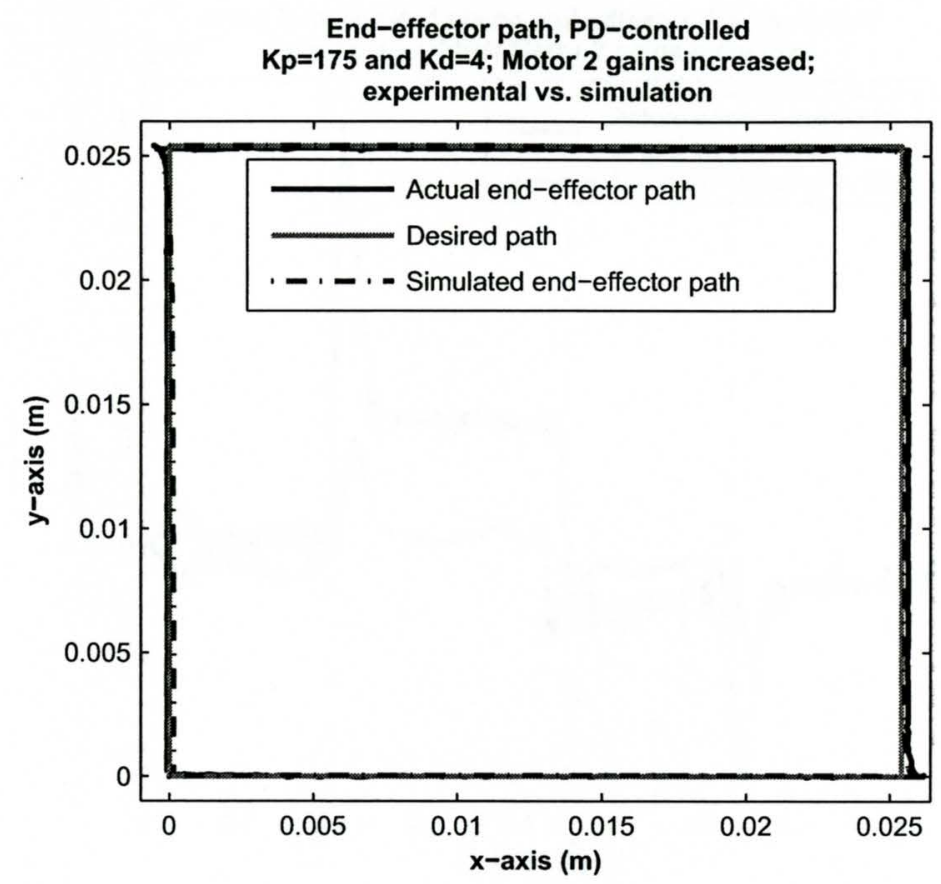

Figure 5.32.

dynamic model. The major deviations are experienced in the offsets. An explanation for that can be found by looking again at the end-effector path derived from the encoder angles assuming rigid belt conditions. Figure 5.33 shows the error in shape of the actual end-effector and the end-effector based on the encoders for the experimental data. It shows that the encoder based end-effector position is very close to the desired position in the steady state cases while traveling along the leg. This suggests that the effect of nonlinear friction in the linear axis combined with a rest stretch in the belt has a bigger effect on the end-effector position on the real system then the simulation suggested, which leads to the deviations between simulation and experimental end-effector path.

To further generalize our findings a couple further tests where carried out. First the length of the legs of the test trajectory was changed to 2 in by 2 in $(0.0508 \mathrm{~m}$ by $0.0508 \mathrm{~m})$. Figure 5.34 shows the end-effector path for simulation and experi- 


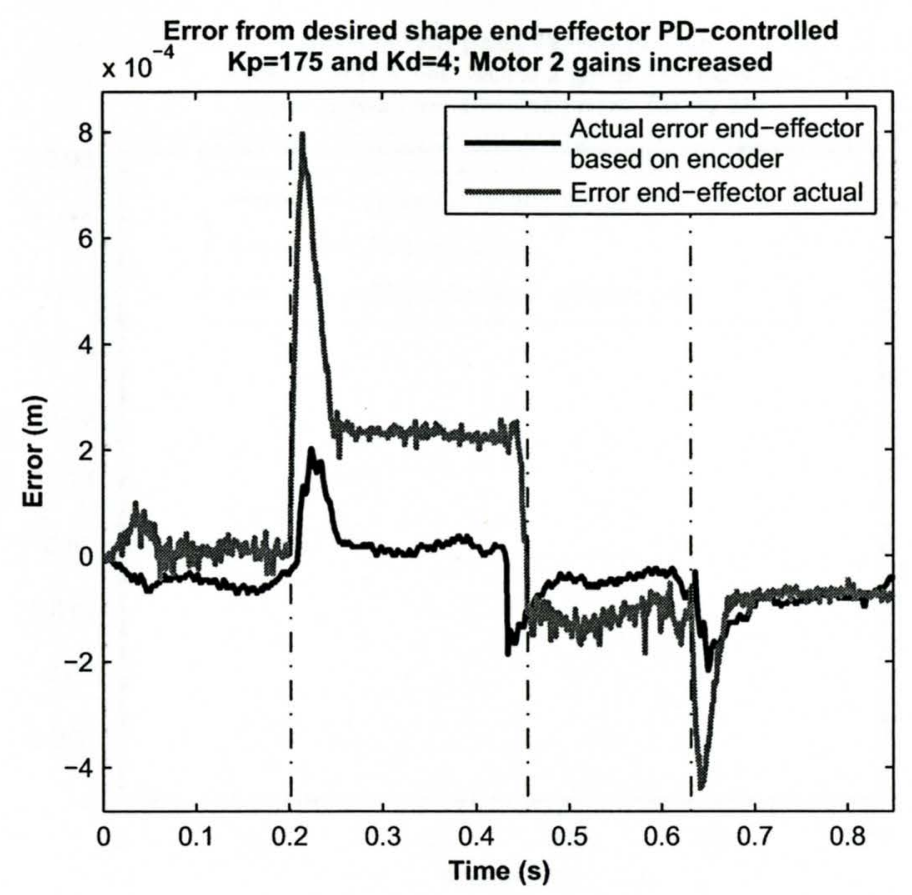

Figure 5.33.

ment for this altered case. No noticeable change of performance can be experienced for this slightly altered case. The first impression, that the overshoots are smaller is explained by the larger scale of this plot.

Another possible alternation in the test trajectory is to change the maximum speed $v_{\max }$. For the next test the length of the legs is reset to 1 in $(0.0254 \mathrm{~m})$, but the speed $v_{\text {max }}$ is changed from $6 \frac{i n}{s}\left(0.1524 \frac{\mathrm{m}}{\mathrm{s}}\right)$ to $3 \frac{\mathrm{in}}{\mathrm{s}}\left(0.0762 \frac{\mathrm{m}}{\mathrm{s}}\right)$. Figure 5.35 shows that as expected the overshoots at the end of the legs decreased, due to slower travel and therefore less acceleration and inertial forces while changing direction. It also shows that since the desired path does not get overshot by the end-effector the nonlinear friction causes the end-effector to stay inside the desired path at all time. The most significant observation in this plot however is the deviation of simulated and experimental end-effector path in the fourth leg. This suggests that as mentioned before the nonlinear friction does have bigger effect on the real 


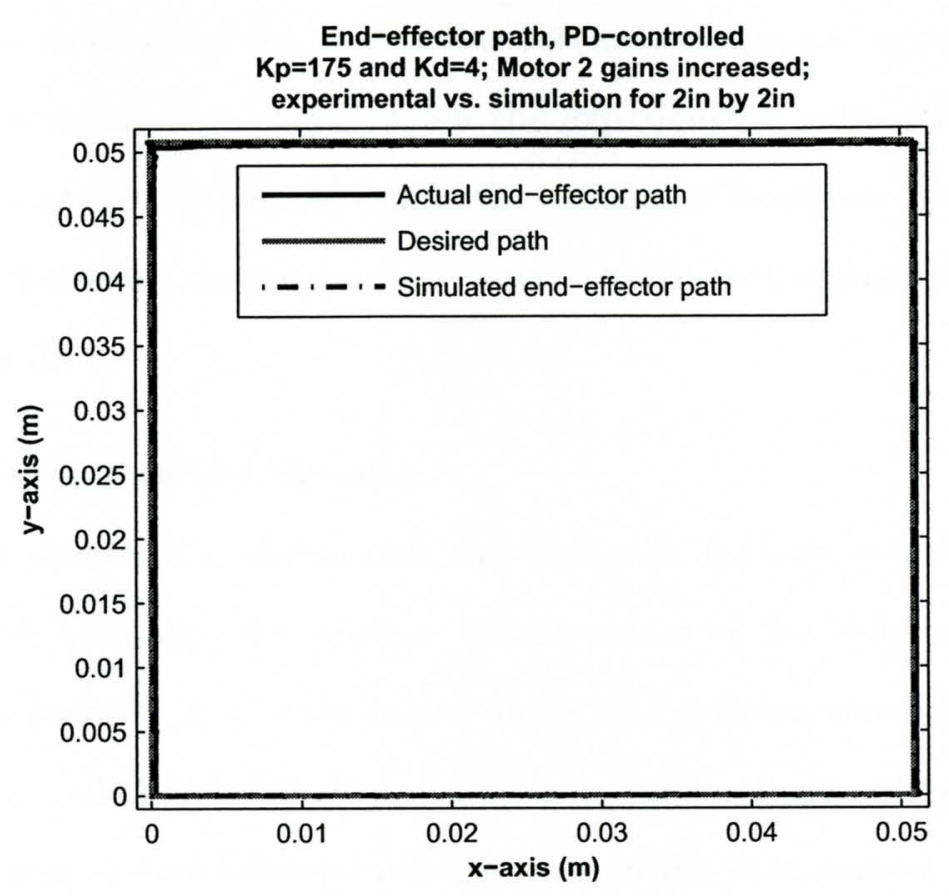

Figure 5.34.

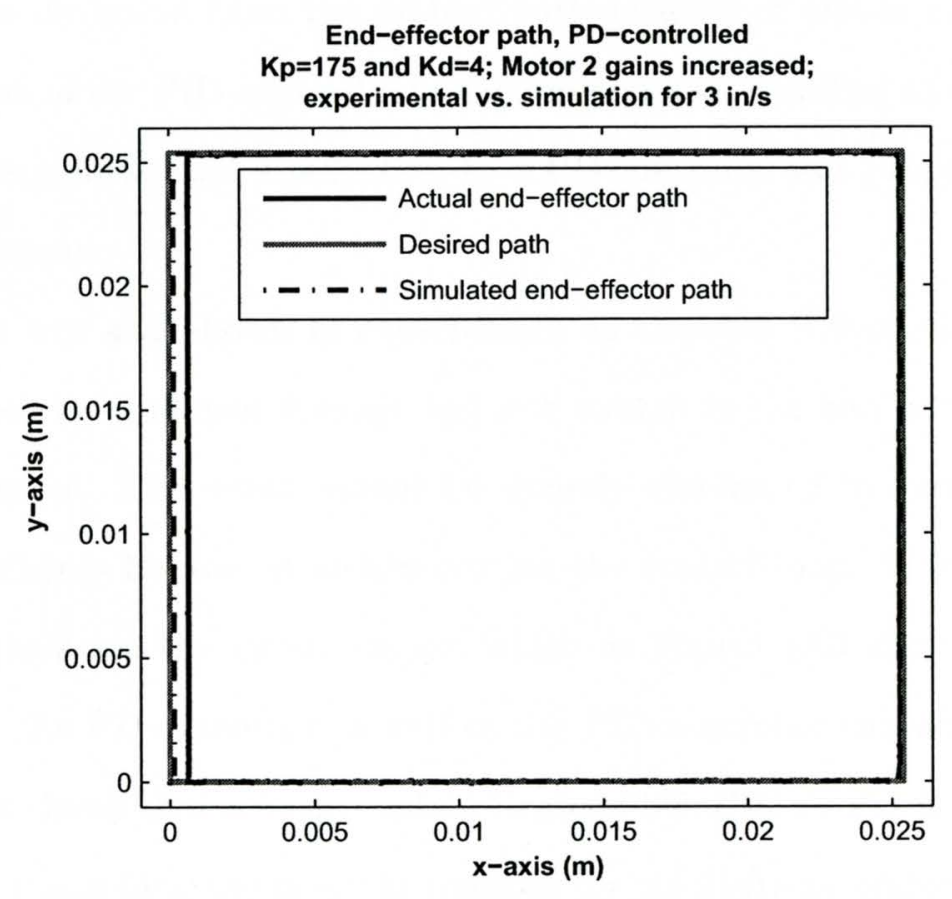

Figure 5.35. 
system than reflected by the model. However taking also into account that for the second leg simulation seems to match the experimental path well, suggests that not only the axes traveling on defines the amount of nonlinear friction. It seems, that also other effects as for instance direction of travel or position seem to have an effect on it.

\subsection{Discussion of the Results}

In this chapter it is shown that the nonlinear dynamic model derived in this thesis is able to match the general characteristics of the $\mathrm{H}$-frame system under closed loop control. Also some minor deviations between simulation and experimental data could be observed. Approaches to further improve the model's reflection of the real system behavior are discussed in the next section.

Furthermore it was shown that unequal motor angle errors are major contributor to the deviation from the desired path in form of offsets in the legs. The integral part of the PID-controller first examined was identified as the major cause of those inequalities and a reduction to a $\mathrm{PD}$-controller was proposed to improve the performance.

However it was also shown in experiments on the real $\mathrm{H}$-frame system that the offset caused by nonlinear friction and rest stretch in the belt sections is a major source of error. This error cannot be entirely eliminated by how the controller is setup. This is because it occurs outside the control loop. Since the feed back control signals are the motor angles, which as Figure 5.33 shows are controlled fairly well, the PD-controller as well as the PID-controller can not eliminate this error. This shows that a basic control strategy like PID or PD is not suitable for accurately controlling the position tracking for the H-frame system. 


\subsection{Further Research}

Based on the findings in this work the following section points out further research and design improvements which can improve the tracking capability of the H-frame system.

The results obtained for the closed loop control of the H-frame system showed that the error caused by the stretch in the timing belt has a large contribution to the overall error. For the system that is assembled in the Robotics Lab it is believed that the relation between moved masses and friction forces and the stiffness of the used timing-belt is not optimal. The use of a stiffer timing belt could improve the results and minimize the error contributed by the elastic transmission element. However to effectively increase the stiffness of the belt, the cross-section-area of the belt would have to be increased, which would also cause a major change of many other elements of the H-frame system like the pulleys. It also has to be understood that this could only minimize the error contributed by the timing-belt, but since a timing-belt will always remain a flexible transmission element the error cannot be completely eliminated.

Also an improvement of the reflection of the real system behavior by the model, derived in this work, is desirable especially if intended to use for sophisticated controller design and implementation.

To further improve the model, differences in the performance of the motors, which were revealed in this work should be further examined and quantified. This, if considered in the model, would enable the model to reflect the real system behavior even more closely. Also a more accurate determination of the friction parameters for coulomb friction as well as for viscous friction experimentally would be desirable. In this work the coefficients were determined through the response of the whole system, which could have lead to an overlapping of effects. The examina- 
tion of the parameters separate from each other could also reveal differences for example in the friction of the two motors, which are currently believed to have the same friction coefficients.

Most importantly though, the model derived in this work can be used to overcome the challenges in the accurate positioning of the $\mathrm{H}$-frame system by utilizing it for the design and implementation of more sophisticated controllers. All those control strategies addressed in the literature review in chapter 1 are potentially suitable for an improvement of the positioning. However the implementation of them for the H-frame system is far from being trivial. This is because all of the papers mentioned in the literature review were working either only with one axis systems, or if considering a xy-positioning, one actuator was always clearly related to a certain movement of the end-effector in either the $\mathrm{x}$ - or $\mathrm{y}$-direction. This is not the case for the H-frame system. The coupling of both actuator movements makes the implementation of those listed control strategies more challenging. However this work, through the derivation of the nonlinear dynamic model, provides future researchers with a tool to design as well as simulate the performance of sophisticated control strategies, that can solve the problems that were pointed out in this work, which will lead to a more accurate control of the $\mathrm{H}$-frame positioning system. 


\section{LIST OF REFERENCES}

[1] H. Lim, J.-W. Seo, and C.-H. Choi, "Position control of xy table in cnc machining center with non-rigid ballscrew," in Proceedings of the American Control Conference, 2000, pp. 1542-1546.

[2] A. Hace, K. Jezernik, B. Curk, and M. Terbuc, "Robust motion control of xy table for laser cutting machine," IEEE, 1998.

[3] A. S. Kulkarni and M. A. El-Sharkawi, "Intelligent precision position control of elastic drive systems," IEEE Transaction on Energy Conversion, vol. 16, no. 1, pp. 26-31, 2001.

[4] A. Hace, K. Jezernik, and A. Sabanović, "Improved design of vss controller for a linear belt-driven servomechanism," IEEE/ASME Transactions on Mechatronics, vol. 10, no. 4, 2005.

[5] T. S. S. Jayawardene, M. Nakamura, , and S. Goto, "Accurate control of belt drives under acceleration and velocity constraints," International Journal of Control, Automation, and Systems, vol. 1, no. 4, 2003.

[6] P. Vedagarbha, D. M. Dawson, and M. Feemster, "Tracking control of mechanical systems in the presence of nonlinear dynamic friction effects," in Proceedings of the American Control Conference, 1997, pp. 2284-2288.

[7] M. Feemster, M. Vedagarbha, D. M. Dawson, and D. Haste, "Adaptive control techniques for friction compensation," Mechatronics, vol. 9, pp. 125-145, 1999.

[8] R. J. Vaccaro, Digital Control: A State-Space Approach. McGraw-Hill, 1995.

[9] J. H. Ginsberg, Advanced Engineering Dynamics, 2nd ed. Cambridge University Press, 1998.

[10] W. J. Palm, System Dynamics. McGraw-Hill, 2005.

[11] W. J. Palm, Modeling, Analysis, and Control of Dynamic Systems, 2nd ed. John Wiley \& Sons, Inc., 2000. 


\section{APPENDIX}

\section{Visual Basic Code for PID-controller with Reset Windup}

The code that generates the PID-controller output for each Motor is given in this Appendix. It is written in Visual Basic syntax in which the controlling program on the $\mathrm{PC}$ controlling the $\mathrm{H}$-frame system is written. The function takes the number of the motor for which the control output is suppose to be computed, the actual encoder position and the desired motor position as inputs and returns the desired controller output. The variables Tsamp, prev_err1, prev_err2, integM1, integM2, kpM1, kpM2, kdM1, kdM2, kiM1 and kiM2 are global variables, which are declared globally in the calling function.

Public Function PIDCont (ByVal vactual As Double,... ByVal vdes As Double, ByVal activemotor As Integer) As Single

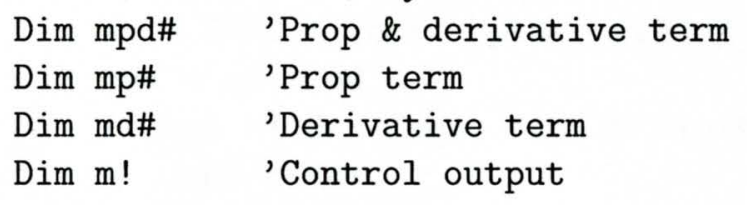

End If

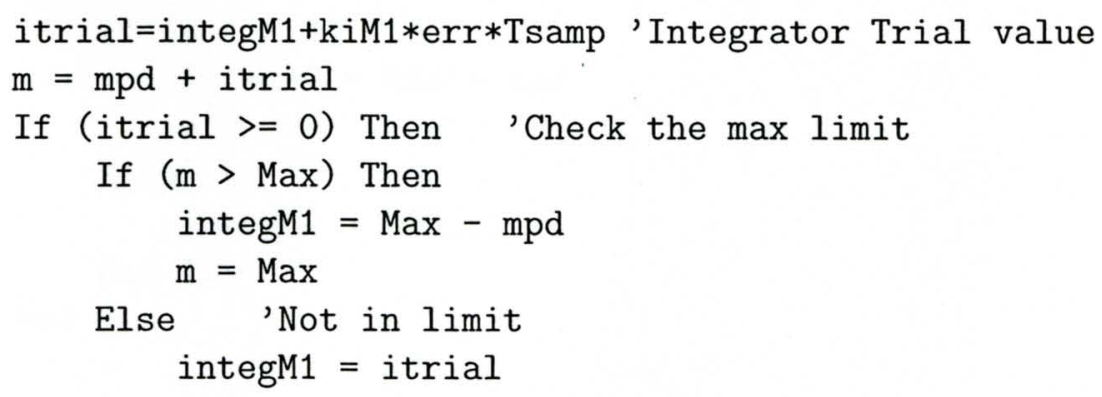


End If

End If

If itrial < 0 Then 'Check the min limit

If $(m<$ Min) Then

integM1 $=$ Min $-\mathrm{mpd}$

$\mathrm{m}=$ Min

Else

integM1 = itrial

End If

End If

prev_err1 = err 'Update prev_err term for next scan

ElseIf activemotor $=2$ Then

$\mathrm{mp}=\mathrm{kpM} 2 *$ err

$\mathrm{md}=\mathrm{KdM} 2 *($ err - prev_err2) / Tsamp

$\mathrm{mpd}=\mathrm{mp}+\mathrm{md}$

If mpd >= Max Then 'apply limits to output $\operatorname{mpd}=\operatorname{Max}$

ElseIf mpd <= Min Then

$\operatorname{mpd}=\operatorname{Min}$

End If

itrial=integM2+kiM2*err*Tsamp 'Integrator Trial value

$\mathrm{m}=\mathrm{mpd}+$ itrial

If (itrial >=0) Then 'Check the max limit

If $(m>$ Max) Then

integM2 $=$ Max $-\operatorname{mpd}$

$\mathrm{m}=\operatorname{Max}$

Else 'Not in limit

integM2 = itrial

End If

End If

If itrial < 0 Then 'Check the min limit

If $(m<$ Min) Then

integM2 = Min $-\mathrm{mpd}$

$\mathrm{m}=$ Min

Else

integM2 = itrial

End If

End If 
prev_err2 $=$ err 'Update prev_err term for next scan End If

PIDCont $=\mathrm{m}$

End Function 


\section{BIBLIOGRAPHY}

Armstrong-Helouvry, B., Dupont, P., and de Wit, C. C., "A survey of models, analysis tools and compensation methods for the control of machines with friction," Automatic, vol. 30, no. 7, pp. 1083-1138, 1994.

Feemster, M., Vedagarbha, M., Dawson, D. M., and Haste, D., "Adaptive control techniques for friction compensation," Mechatronics, vol. 9, pp. 125-145, 1999.

Ginsberg, J. H., Advanced Engineering Dynamics, 2nd ed. Cambridge University Press, 1998.

Hace, A., Jezernik, K., Curk, B., and Terbuc, M., "Robust motion control of xy table for laser cutting machine," IEEE, 1998.

Hace, A., Jezernik, K., and Sabanović, A., "Improved design of vss controller for a linear belt-driven servomechanism," IEEE/ASME Transactions on Mechatronics, vol. 10, no. 4, 2005.

Jayawardene, T. S. S., Nakamura, M., , and Goto, S., "Accurate control of belt drives under acceleration and velocity constraints," International Journal of Control, Automation, and Systems, vol. 1, no. 4, 2003.

Kim, B. K., Park, S., Chung, W. K., and Youm, Y., "Robust controller design for ptp motion of vertical xy positioning systems with flexible beam," IEEE/ASME Transactions on Mechatronics, vol. 8, no. 1, 2003.

Kim, J.-J. and Singh, T., "Controller design for flexible systems with friction: Pulse amplitude control," AIAA Guidance, Navigation and Control Conference, 2004.

Kulkarni, A. S. and El-Sharkawi, M. A., "Intelligent precision position control of elastic drive systems," IEEE Transaction on Energy Conversion, vol. 16, no. 1, pp. 26-31, 2001.

Lim, H., Seo, J.-W., and Choi, C.-H., "Position control of xy table in cnc machining center with non-rigid ballscrew," in Proceedings of the American Control Conference, 2000, pp. 1542-1546.

Lin, L.-C. and Lin, Y.-J., "Fuzzy-enhanced adaptive control for flexible drive system with friction using genetic algorithm," Journal of Intelligent and Robotic Systems, vol. 23, no. 2, pp. 379-405, 1998. 
Makkar, C., Dixon, W. E., Sawyer, W. G., and Hu, G., "A new continuously differentiable friction model for control system design," in Proceedings of the 2005 IEEE/ASME International Conference on Advanced intelligent Mechatronics, 2005, pp. 600-605.

Palm, W. J., Modeling, Analysis, and Control of Dynamic Systems, 2nd ed. John Wiley \& Sons, Inc., 2000.

Palm, W. J., System Dynamics. McGraw-Hill, 2005.

Singru, P. M. and Modak, J. P., "Computer simulation of the dynamic and vibration response of a belt drive pulley," Journal of Sound and Vibration, pp. 277-293, 2001.

Tseng, Y.-T. and Liu, J.-H., "High-speed and precise positioning of an x-y table," Control Engineering Practice, vol. 11, 2003.

Vaccaro, R. J., Digital Control: A State-Space Approach. McGraw-Hill, 1995.

Vedagarbha, P., Dawson, D. M., and Feemster, M., "Tracking control of mechanical systems in the presence of nonlinear dynamic friction effects," in Proceedings of the American Control Conference, 1997, pp. 2284-2288. 